\title{
REVIEW ARTICLE OPEN Roles and mechanisms of exosomal non-coding RNAs in human health and diseases
}

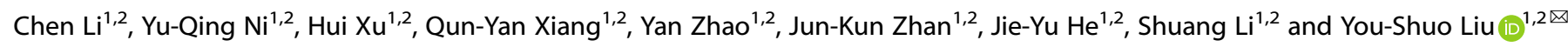

Exosomes play a role as mediators of cell-to-cell communication, thus exhibiting pleiotropic activities to homeostasis regulation. Exosomal non-coding RNAs (ncRNAs), mainly microRNAs (miRNAs), long non-coding RNAs (IncRNAs), and circular RNAs (circRNAs), are closely related to a variety of biological and functional aspects of human health. When the exosomal ncRNAs undergo tissuespecific changes due to diverse internal or external disorders, they can cause tissue dysfunction, aging, and diseases. In this review, we comprehensively discuss the underlying regulatory mechanisms of exosomes in human diseases. In addition, we explore the current knowledge on the roles of exosomal miRNAs, IncRNAs, and circRNAs in human health and diseases, including cancers, metabolic diseases, neurodegenerative diseases, cardiovascular diseases, autoimmune diseases, and infectious diseases, to determine their potential implication in biomarker identification and therapeutic exploration.

Signal Transduction and Targeted Therapy (2021)6:383

; https://doi.org/10.1038/s41392-021-00779-x

\section{INTRODUCTION}

Exosomes are a class of extracellular vesicles (EVs) $\sim 30-150 \mathrm{~nm}$ in diameter. ${ }^{1,2}$ Exosomes were first reported in sheep reticulocytes in 1983. Further studies reported that exosomes are derived from most cell types and are present in the cell-conditioned medium and distinct biological fluids such as serum, plasma, urine, saliva, ascites, cerebrospinal fluid, and amniotic fluid. ${ }^{3}$ Exosomes were initially regarded as means of cellular waste disposing until further studies reported their role in mediating cell-to-cell communication, thus attracting significant attention of scholars worldwide. ${ }^{4,5}$ Several functions of exosomes have been characterized including cellular proliferation, differentiation, apoptosis, angiogenesis, and immune regulation. ${ }^{6,7}$ Exosomes exhibit these functions by interacting with surface receptors of recipient cells thus transmitting biomolecules such as lipids, proteins, messenger RNAs (mRNA), and non-coding RNAs (ncRNAs) to recipient cells. ${ }^{8}$ Notably, ncRNAs are the components of exosomes that have attracted particular attention. ${ }^{9}$

NcRNAs refer to molecules that lack protein-coding regions, which have become a hot topic of increasing concern. The correlation of ncRNAs with human diseases has primarily been identified in the function and expression of miRNA found in cancers. However, the extent of ncRNAs involvement in diseases is only just being explored. To better understand the discovery and research history of ncRNAs in human health and diseases, it is helpful to review the timeline of ncRNAs (Fig. 1). Discovery of housekeeping ncRNAs, such as ribosomal RNA (rRNA) ${ }^{10}$ and transfer RNA (tRNA) ${ }^{11}$ in the 1950s, supported Crick's "central dogma" theory, which stated that genetic information can proceed from DNA to RNA to protein. ${ }^{12}$ Further studies reported new ncRNAs, such as small nuclear RNAs (snRNA), ${ }^{13,14}$ small nucleolar RNAs (snoRNA) ${ }^{15,16}$ and circular RNAs (circRNAs). ${ }^{17}$ In the late 1980s, studies first reported long non-coding RNAs (IncRNAs), such as $\mathrm{H}_{19^{18}}$ and Xist. ${ }^{19,20}$ The human genome sequence was published in 2001, ${ }^{21}$ and the findings showed that genes that encoded proteins only accounted for $1.2 \%$ of the genome, whereas the rest were considered as "non-coding". ${ }^{22}$ The first small temporal RNAs, lin-4, ${ }^{23}$ and let- $7,{ }^{24}$ were discovered in Caenorhabditis elegans in 1993 and 2000, respectively. Analysis showed that ncRNA can act as conserved functional molecules needed for development. Further studies showed that large numbers of gene do not encode proteins but encode various unique transcripts. ${ }^{25-28}$ NcRNAs can regulate gene expression at transcriptional, post-transcriptional, and translational levels, thereby modulating associated signaling networks. ${ }^{29}$ In addition, different kinds of ncRNAs can interact with each other to regulate their stability or abundance. ${ }^{30}$ In 2007, it was reported that exosomes contain microRNAs (miRNAs) and mRNA and can transfer them to other cells. ${ }^{31}$ Since then, accumulating evidence demonstrates that a variety of ncRNAs can be encapsulated and transported by exosomes, the most attractive of which are miRNAs, IncRNAs, and circRNAs, explaining their roles in intercellular communication. ${ }^{32,33}$ Notably, exosomal ncRNAs exhibit diverse expression patterns in different cells or various physiological and pathological conditions, indicating the potential role of these exosomal biomolecules in occurrence and development of different diseases. ${ }^{34,35}$ These differences in expression levels in pathological states indicate that exosomal ncRNAs are promising diagnostic and therapeutic tools for various human diseases. ${ }^{9,36-38}$ A large number of studies dealing with circulating exosomes and their cargoes prove that exosomal miRNAs, IncRNAs and circRNAs are closely involved in human health and the initiation and development of various diseases. ${ }^{39}$ Therefore, we specifically focus on effects of exosomal ncRNAs (miRNAs,

\footnotetext{
${ }^{1}$ Department of Geriatrics, The Second Xiangya Hospital, Central South University, Changsha, Hunan 410011, China and ${ }^{2}$ Institute of Aging and Age-related Disease Research, Central South University, Changsha, Hunan 410011, China

Correspondence: You-Shuo Liu (liuyoushuo@csu.edu.cn)

These authors contributed equally: Chen Li, Yu-Qing Ni
}

Received: 1 May 2021 Revised: 23 September 2021 Accepted: 26 September 2021

Published online: 10 November 2021 


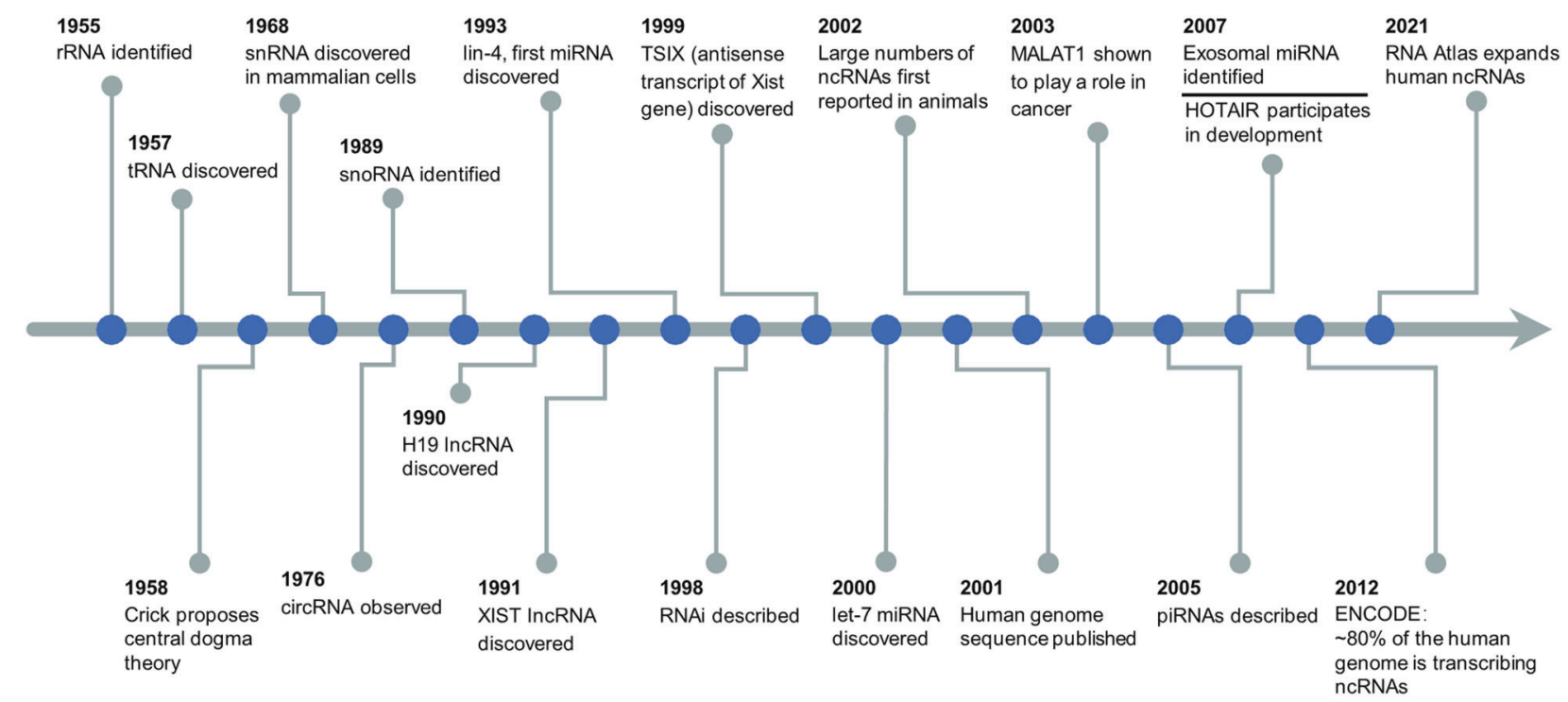

Fig. 1 Timeline of the discovery and research history of ncRNAs in human health and diseases. Key discoveries are highlighted. XIST X (inactive)-specific transcript, RNAi RNA interference, piRNA PIWI-interacting RNA, HOTAIR HOX transcript antisense RNA, ENCODE Encyclopedia of DNA Elements

IncRNAs, and circRNAs) in physiopathology, clinical diagnosis, and therapy of human diseases, such as metabolic diseases, cancers, neurodegenerative diseases, cardiovascular diseases, autoimmune diseases, and infectious diseases.

\section{POTENTIAL REGULATORY MECHANISMS OF EXOSOMES IN HUMAN DISEASES}

Several studies reported the importance of exosomes in human health and diseases. ${ }^{40,41}$ In addition, recent studies demonstrated that exosomes could exert their roles by modulating immune response, oxidative stress, autophagy, gut microbe, and cell cycle. $^{42-45}$ This section provides a comprehensive overview of the current understanding of exosomes research and discusses potential mechanisms of exosomes in human diseases (Fig. 2).

\section{Immune response}

Exosomes released from immune and non-immune cells exert a pivotal effect in immune regulation. ${ }^{46-48}$ Recent studies reported the functions of exosomes in triggering or inhibiting immune response, indicating their potential roles in the development and progression of autoimmune and inflammatory diseases. ${ }^{49-54}$

Exosomes can regulate immune response via the transfer and presentation of antigenic peptides. Exosomes derived from antigenpresenting cells (APCS) activate T cells by carrying major histocompatibility complex class II (MHC-II) that binds to antigenic peptides. ${ }^{48}$ Notably, APC-released exosomes with MHC-II bearing tumor peptides, significantly inhibit tumor growth in mice in a $\mathrm{T}$ cell-dependent manner. ${ }^{55}$ In addition, exosomes from APCs carrying bacterial antigens promote activation of anti-bacterial immunity. For instance, macrophage-derived exosomes carrying mycobacterial antigens protect mice against mycobacterium tuberculosis infection by inducing $\mathrm{CD}^{+}$and $\mathrm{CD}^{+}$T cells to produce IFN- $\gamma$ and $\mathrm{IL}-2 .^{56}$ However, excessive immune response mediated by exosomes can cause normal tissue damage thus promoting onset and development of diseases. For example, circulating exosomes from patients with Hashimoto thyroiditis can present antigens to dendritic cells (DCs), thus inducing DC activation through the NF-KB signaling pathway, contributing to imbalanced differentiation in $\mathrm{CD}^{+} \mathrm{T}$ cells, and potentially leading to Hashimoto thyroiditis onset. ${ }^{54}$

Exosomes can mediate tumor or pathogen immune escape by affecting gene expression in immune cells, mainly by delivering
miRNAs, thus promoting progression of cancers and infection. For instance, tumor-derived exosomal miR-212-3p downregulates expression of regulatory factor X-associated protein (RFXAP), which inhibits MHC-II and promotes immune tolerance of dendritic cells. ${ }^{57}$ Moreover, virus-infected cell can transmit viral miRNAs to uninfected host immune cells through exosomes, thus downregulating immunomodulatory genes. ${ }^{58}$ Therefore, exosomes present a novel intersection between immune response and disease.

Moreover, exosomes seem to be involved in the regulation of macrophage and neutrophil polarization, which ultimately can induce the pathophysiologic process of several diseases. Macrophages comprise a population of heterogeneous cells which are classified into two classes including pro-inflammatory M1 or antiinflammatory M2 macrophages based on their activation status. ${ }^{59}$ Exosomes can induce macrophage differentiation into $\mathrm{M} 1$ or $\mathrm{M} 2$ phenotypes, a critical regulatory mechanism of inflammation, which has essential effects on homeostasis. ${ }^{60-63}$ For example, adipose-derived exosomes exacerbate insulin resistance (IR) and atherosclerosis by inducing macrophage M1 polarization. ${ }^{62,64}$ Moreover, exosomes derived from cancer cells can also promote M1 macrophage polarization. Xiao and coworkers reported that exosomal thrombospondin-1 (THBS1) secreted by oral squamous cell carcinoma cells can activate M1 macrophages polarization to promote malignant migration. ${ }^{65}$ Notably, exosomes can induce activation of $\mathrm{M} 2$ macrophages thus inhibiting inflammatory response, leading to abrogation of many diseases. ${ }^{66,67}$ Studies reported that exosomes from mesenchymal stem cells (MSCs) ameliorate cardiac damage in myocardial infarction rats and ischemia/reperfusion mice by activating macrophage $M 2$ polarization. $^{68,69}$ However, M2 macrophages polarization can be detrimental. For example, M2 macrophages support tumor growth and survival. ${ }^{70}$ Studies report that tumor-derived exosomes promote occurrence and development of tumor by activating $\mathrm{M} 2$ phenotypes. Exosomal DLX6-AS1 from HCC cells triggers M2 macrophage polarization to provoke tumor invasion and migration through the miR-15a-5p/CXCL17 axis. ${ }^{71}$ Besides, exosomes can play a crucial role in disease progression by inducing neutrophil polarization. ${ }^{72,73}$ Laboratory tests have confirmed the existence of N1 (antitumoral) and N2 (protumoral) tumor-related neutrophils, parallel to $M 1$ and $M 2$ macrophage polarization. ${ }^{74}$ Moreover, tumor-derived exosomes can induce N2 polarization of neutrophils to promote cancer progression. ${ }^{72}$ Conversely, 


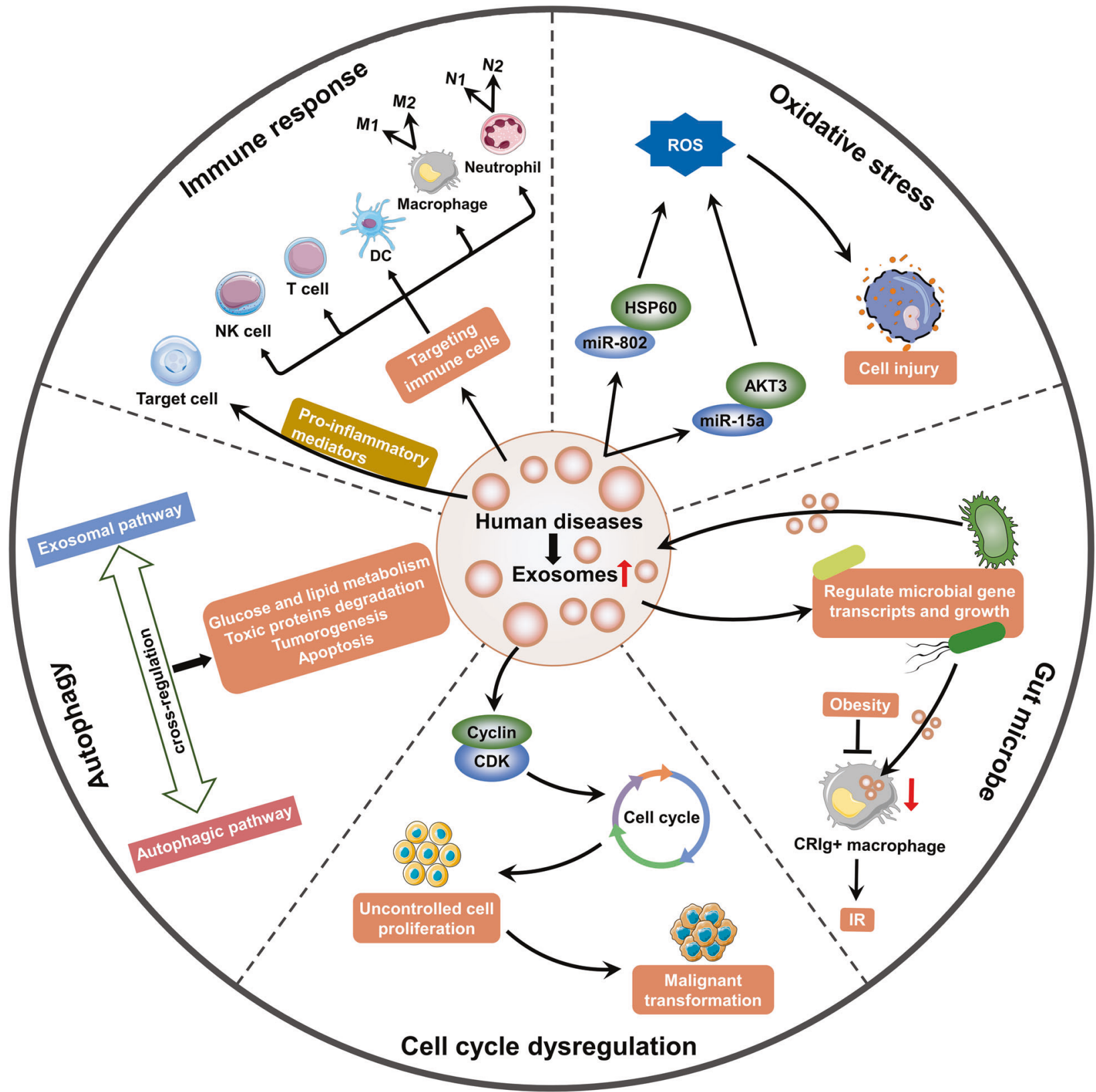

Fig. 2 The potential regulatory mechanisms of exosomes in human diseases. Several mechanisms of the occurrence of human diseases are modulated by exosomes, including immune response, oxidative stress, autophagy, gut microbe, and cell cycle. This figure was created with the aid of Servier Medical Art (https://smart.servier.com/). ROS reactive oxygen species, HSP heat shock protein, AKT3 AKT serine/threonine kinase 3, IR insulin resistance, CDK cyclin-dependent kinase, NK natural killer, DC dendritic cell

exosomes that inhibit neutrophil inflammatory response can alleviate the tissue injury and have the therapeutic potential. ${ }^{75}$

In addition, exosomes can modulate immune response by transporting cytokines or other pro-inflammatory mediators thus directly acting on target organs. ${ }^{76-78}$ Macrophage-derived exosomes containing miR-21-5p promote inflammatory activation and regulate podocyte injury in diabetic nephropathy mice. ${ }^{79}$ Fabbri et al. found that tumor-secreted exosomal miRNAs induce inflammatory response that may contribute to tumor growth and metastasis. ${ }^{80}$ Conversely, some exosomes exert anti-inflammatory effects that can be targeted for development of therapies. ${ }^{81}$ For instance, exosomal miR-192 significantly attenuates tumor metastasis by suppressing secretion of proangiogenic factors, such as interleukin (IL)-8, intercellular cell adhesion molecule (ICAM), and C-X-C motif chemokine ligand 1 (CXCL1). ${ }^{82}$
Collectively, exosomes have been identified to regulate immune responses by carrying biomolecules to targeted cells, thereby affecting the phenotype and immunomodulatory functions of immune cells, or directly acting on target organs. Particularly, exosomes derived from immune cells or nonimmune cells exert pivotal roles in immunotherapy. In this section, we discuss the roles of exosomes as carriers for regulating immune responses and as predictive biomarkers for immune activation.

\section{Oxidative stress}

Oxidative stress refers to an imbalance in oxidative-antioxidative systems and leads to excessive accumulation of reactive oxygen species (ROS), which contributes to various disorders by inducing cell and tissue dysfunction. 
Nutrition stress promotes oxidative stress. ${ }^{83}$ Advanced glycation end-products (AGEs) play an important role in ROS production and promote oxidative stress in diabetes individuals. ${ }^{84}$ Exosomes exhibit an essential role in oxidative stress. Exosomal miR-802-5p derived from hypertrophic adipocyte causes cardiac IR by suppressing expression of heat shock protein 60 (HSP60), which is implicated in promoting oxidative stress. ${ }^{85}$ Kamalden and coworkers reported that pancreatic $\beta$-cells-derived exosomal miR15 a can travel through the circulation and induce oxidative stress by targeting AKT serine/threonine kinase 3 (AKT3), leading to retinal injury in T2DM subjects. ${ }^{43}$ Furthermore, exosomal-miR-21$5 p$ from macrophages induces podocyte injury in diabetic nephropathy mice partially by promoting ROS production. ${ }^{79}$ In addition, oxidative stress is associated with age-related bone loss that increases the risk of osteoporosis. ${ }^{86}$ Exosomes from serum of aged normal individuals can exert a protective effect on bone health by inhibiting aging-related oxidative stress. ${ }^{87}$ Recent studies have documented that exosomal circHIPK3 produced by hypoxia-pretreated cardiomyocytes decreases oxidative stressinduced cardiac microvascular endothelial cells dysfunction. ${ }^{88}$

To sum up, these findings indicate the relationship between exosomes and oxidative stress in human disease. Exosomes have been shown to play a vital part in modulation of oxidative stress during the occurrence and development of various diseases.

\section{Autophagy}

Under normal physiological conditions, autophagy serves as a protective mechanism to remove protein aggregates, impaired organelles, and invading pathogens, and is implicated in recycling amino acids, lipids, and sugars to maintain cellular renovation and homeostasis. ${ }^{89,90}$ However, autophagy dysfunction is associated with several diseases, such as cancers, neurodegenerative diseases, and metabolic diseases. The crosstalk between autophagy and exosome biogenesis varies with the type of disease. ${ }^{91}$ Autophagy can reduce release of exosomes through multivesicular bodies (MVB) degradation. ${ }^{92}$ In addition, exosome release and autophagy can act synergistically against cell stress. ${ }^{93}$

Reduced autophagy level is reported in multiple diseases, such as obesity, hyperglycemia, and osteoporosis..$^{90,94-96} \mathrm{Li}$ and colleagues revealed that defective autophagy induced by adipose sirtuin 1 (SIRT1) deficiency increases exosome release from adipose tissue, thus promoting decreased glucose tolerance, diminished insulin sensitivity, and impaired lipid metabolism. ${ }^{97}$ Mammalian sterile 20like kinase 1 (Mst1)-enclosed exosomes from cardiac microvascular endothelial cells (ECs) are implicated in inhibition of autophagy, promotion of apoptosis and suppression of glucose uptake in diabetic cardiomyopathy. ${ }^{98}$ Notably, exosomes play a cell protective role by activating intracellular autophagy. ${ }^{91}$ MSC transplantation after myocardial infarction improves cardiac function and infarct size partially through release of exosomes that improve autophagic flux. Accumulating evidence indicates that MSC-derived exosomes alleviate T2DM complications including diabetic ulcers and nephropathy by inducing autophagy. ${ }^{44,99-101}$ A similar effect is reported in exosomes released from M2 macrophage. ${ }^{102}$

Neuronal cells can eliminate protein aggregates to ameliorate proteotoxicity through autophagic degradation and exosome release. Conversely, abnormal accumulation and aggregation of proteins are manifestations of various neurodegenerative diseases. Exosome secretion can be elevated to attenuate the toxic proteins during autophagic or lysosomal dysfunction. For instance, Yang and coworkers identified secretory carrier membrane protein 5 (SCAMP5) as an autophagy inhibitor that promote exosomal secretion of alpha-synuclein (a-SYN). ${ }^{103}$

Studies report that cancer cell-derived exosomes affect autophagy in recipient cells. ${ }^{104}$ In addition, exosome regulate drug resistance and tumor microenvironment in an autophagydependent manner. ${ }^{105}$ For instance, exosomal circ-PVT1 promotes cisplatin resistance in gastric cancer cells by inducing cell autophagy and invasion and inhibiting apoptosis. ${ }^{106}$ Furthermore, gastric cancer cell-derived exosomes trigger autophagy and promote activation of neutrophils, ultimately promoting gastric cancer cell migration. ${ }^{72}$

Altogether, these findings indicate that exosomes play important roles in multiple physiological and pathological processes by regulating autophagy. Besides, the biogenesis and release of exosomes are closely associated with autophagy in diseases. Autophagy dysfunction is one of the important potential mechanisms of exosomes in many diseases, such as metabolic diseases, neurodegenerative diseases, and cancers.

\section{Gut microbe}

Gut microbes exert important roles in physiological processes, such as providing essential nutrients, assisting in cellulose digestion, regulating integrity of gut barriers and immune response. ${ }^{107}$ However, dysbiosis, the imbalance in microbiota composition and diversity, in response to internal changes or external stimuli is associated with several chronic diseases, such as autoimmune, metabolic, cardiovascular diseases, and cancer. ${ }^{108-110}$ Intestinal homeostasis relies on dynamic and coordinated interactions between microbes, epithelium, and host immune system. Accumulating evidence has supported that exosomes provide a link between the host and gut microbial community. Liu and coworkers uncovered a new effect of fecal exosomal miRNAs on shaping gut microbiota. Intestinal ECsderived miRNAs-containing exosomes can enter bacteria and regulate gene transcripts and growth, and their loss leads to dysbiosis and aggravation of colitis. ${ }^{111}$ These findings indicate the key roles of fecal exosomal miRNAs on maintaining normal gut microbiota.

Exosomes from beneficial microbes can improve metabolic functions through various mechanisms. Recent studies report that exosomes can restore intestinal and metabolic homeostasis in HFD-induced obesity mice by reversing the adverse effects of obesity including adipose and gut inflammation, intestinal mucosal barrier permeability, and fat weight gain. ${ }^{45,112}$ Conversely, maleficent bacteria is implicated in impairing of metabolic homeostasis. Stool exosomes from Pseudomonas panacis in HFD mice promote glucose intolerance and IR in healthy mice. ${ }^{113}$ Liver $\mathrm{CRIg}^{+}$(complement receptor of the immunoglobulin superfamily) macrophage can clear bacteria and their products from the bloodstream. A recent study reported a decrease in $\mathrm{CRIg}^{+}$ macrophage population in obese subjects. As a result, less circulating gut microbial DNA-containing exosomes were eliminated, and more exosomes diffused to distant metabolic tissues, thus aggravating tissue inflammation and $\mathrm{IR}^{114}$ In addition, gut microbiota are involves in modulation of bone metabolism. A recent study reported that gut microbiota in children and Akkermansia muciniphila release exosomes to bone tissues to ameliorate osteoporosis via promoting osteogenic activity and decreasing osteoclast formation. ${ }^{115}$

With the relevant cumulative findings, we summarize and indicate the role of exosomes as a link between gut microbiota and diseases. Moreover, these findings suggest that the effects of exosomes on the microbiome may be utilized to target specific host processes to ameliorate diseases.

\section{Cell cycle dysregulation}

Cell proliferation and division are basic cell physiological activities. Growth factors, hormones, and oncogene products can induce or inhibit cell proliferation, thereby influencing the cell cycle. Dysregulation of the cell cycle is associated with multiple diseases. Accelerated cell cycle can lead to carcinogenesis. ${ }^{116}$ Recent studies have revealed the essential role of exosomes in regulating cell cycle. Exosomal circRNA_100284 accelerates cell cycle and promotes proliferation by targeting miR-217, which induces malignant transformation of human hepatic cells. ${ }^{117}$ In addition, 


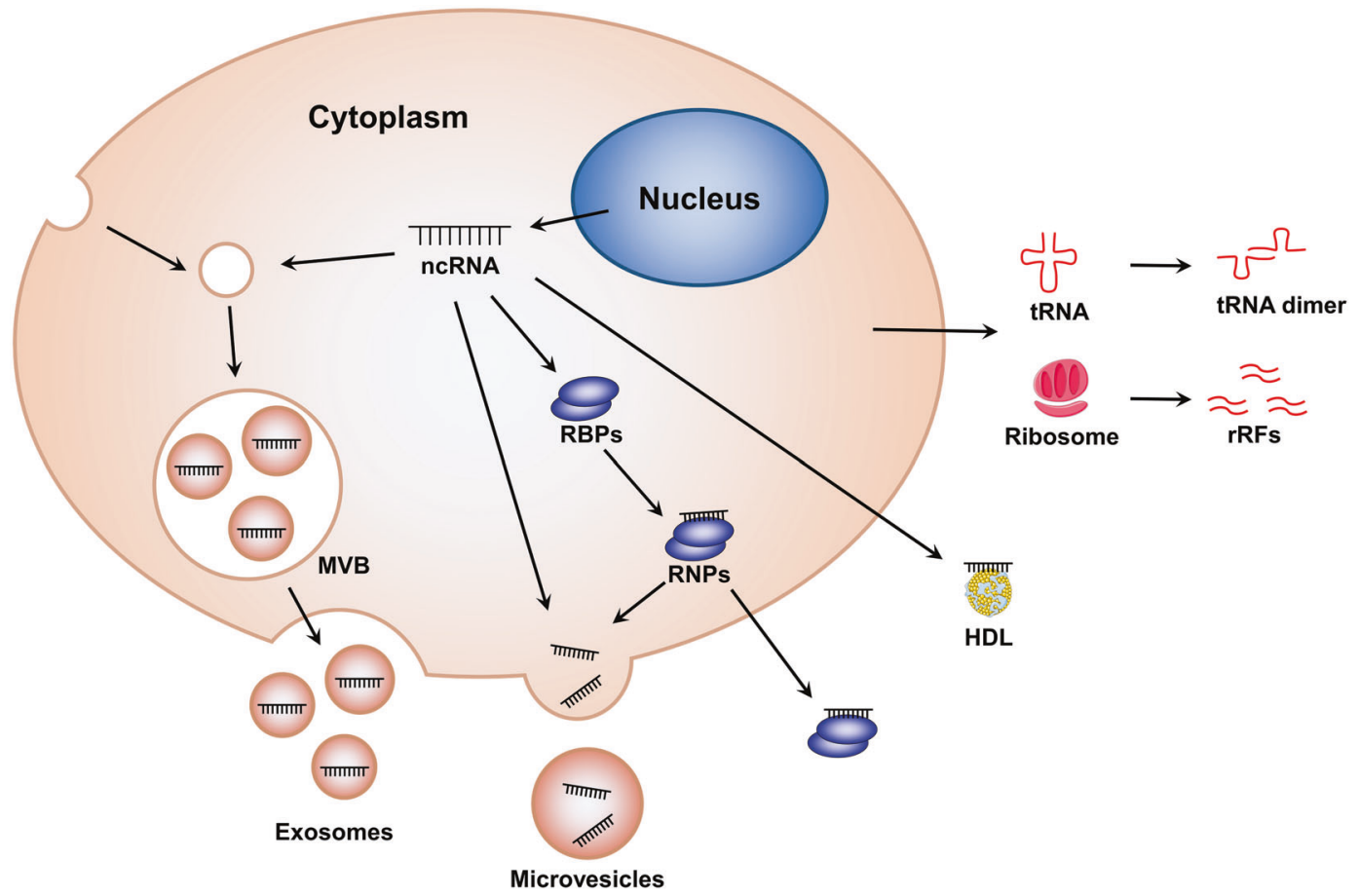

Fig. 3 Different mechanisms underlying the stability of extracellular ncRNAs. NcRNAs can be protected from harsh extracellular environment through extracellular vesicles encapsulation (such as exosomes and microvesicles), ribonucleoprotein (RNP) complex formation, and highdensity lipoprotein (HDL) transportation. Moreover, some extracellular RNA fragments that generate from non-vesicular ncRNAs in extracellular space can form self-protecting dimers. The source of these non-vesicular RNAs remains uncertain. This figure was created with the aid of Servier Medical Art (https://smart.servier.com/). MVB multivesicular bodies, RBPs RNA-binding protein, rRFs rRNA-derived fragments

exosomal IncRNA ZFAS1 promotes gastric cancer progression by shortening cell cycle and epithelial-mesenchymal transition (EMT). ${ }^{118}$ Conversely, exosomes that block cell cycle in cancer can serve as therapeutic targets. ADSC-released exosomal-miRNAs present inhibitory effects on ovarian cancer cells by blocking cell cycle and inducing apoptosis signaling. ${ }^{119}$ These findings show the dual effects of exosomes on cell cycle regulation in the initiation and progression of cancers.

Differences of exosomal and non-exosomal ncRNAs in health and diseases

It has been confirmed that ncRNAs exist not only in cells, but also in different body fluids such as serum, plasma, urine, saliva, and so on. The ncRNAs in biofluids are often referred to as extracellular ncRNAs or circulating ncRNAs. Notably, the RNase activity is high in the extracellular environment, but extracellular ncRNAs remain relatively stable in plasma, suggesting that circulating ncRNAs may be protected and circumvented from harsh conditions. One intriguing mode of transport of circulating ncRNAs is related to exosomes. In this section, we try our best to investigate the differences between ncRNAs in exosomes and non-exosomes in regulating physiological homeostasis and pathological processes in health and diseases (Fig. 3).

NcRNAs can be encapsulated by EVs (including exosomes, microvesicles, and apoptotic bodies) and secreted out of cells to act as mediators for intercellular communication, thereby regulating different diseases according to the target cells. In addition to the vesicle-dependent pattern, a considerable number of extracellular ncRNAs are present in the form of ribonucleoprotein (RNP) complexes with RNA-binding proteins (RBPs) such as argonaute-2 (AGO2) that modulate mRNA inhibition in cells. ${ }^{120,121}$ These RBPs can affect RNA sorting into EVs in an indirect manner. ${ }^{122}$ Moreover, high-density lipoprotein (HDL) can also transport endogenous ncRNAs to recipient cells with functional targeting capabilities, the effect of which may vary depending on the disease state. ${ }^{123}$ Further, recent studies have revealed a postrelease processing of ncRNAs. Some extracellular RNA fragments that generate from non-vesicular ncRNAs, such as ribosomes and full-length tRNAs, in extracellular space can form self-protecting dimers to resist RNases. ${ }^{124,125}$

\section{THE ROLES OF EXOSOMAL NCRNAS IN HUMAN DISEASES}

Accumulating data suggest that exosomal ncRNAs exert pleiotropic effects on human diseases. ${ }^{126,127}$ Among these ncRNAs cargoes, the most intriguing ones are miRNAs, IncRNAs, and circRNAs. MiRNAs are small, highly conserved ncRNAs. ${ }^{128}$ LncRNAs are poorly conserved ncRNAs with a length of more than 200 nucleotides. ${ }^{30}$ CircRNAs are a subset of ncRNAs with covalently closed structures which are implicated in the regulation of gene expression. ${ }^{129}$ In this section, the roles of exosomal miRNAs, IncRNAs, and circRNAs in different human diseases (including cancers, metabolic diseases, cardiovascular diseases, neurodegenerative diseases, autoimmune diseases, and infectious diseases) are explored (Fig. 4).

The roles of exosomal ncRNAs in cancers

GLOBOCAN statistics report that approximately 14.1 million new cancer cases were diagnosed and 8.2 million deaths occurred in 2012. Prevalence of cancer is raising owing to the increase in population growth and aging population, creating a huge health burden for both patients and society. ${ }^{130}$ Recent studies have explored tumorassociated exosomal ncRNAs. ${ }^{131}$ Emerging studies revealed that exosomal ncRNAs are implicated in progression of human cancers, such as lung cancer, ${ }^{132}$ breast cancer (BC), ${ }^{133}$ and hepatocellular carcinoma (HCC). ${ }^{134}$ Exosomal ncRNAs play a role in cancers, including EMT, proliferation, angiogenesis, metastasis, drug resistance, and immune-inflammation (Fig. 5). In this section, the roles of exosomal miRNAs, IncRNAs, and circRNAs in various human cancers, including lung cancer, BC, HCC, colorectal cancer (CRC), gastric cancer (GC), and prostate cancer $(\mathrm{PCa})$ are summarized. 


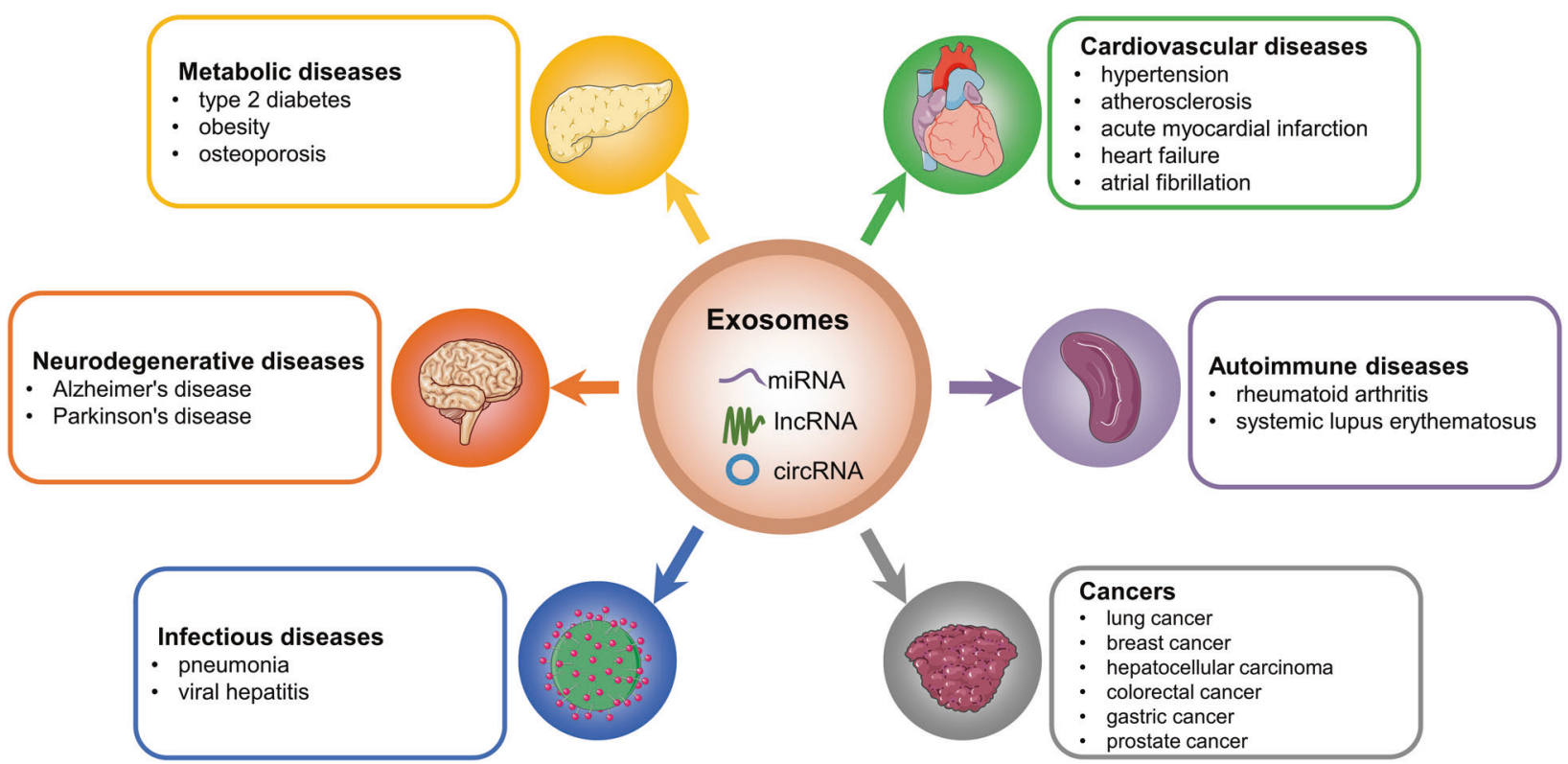

Fig. 4 The roles of exosomal ncRNAs in human diseases. The figure showed examples of human diseases where exosomal ncRNAs exert pivotal function. This figure was created with the aid of Servier Medical Art (https://smart.servier.com/)

Exosomal ncRNAs in lung cancer. Lung cancer is the leading cause of cancer-related mortality globally. Approximately $70 \%$ of lung cancer patients present with complex complications at the time of diagnosis and surgical resection is the primary treatment option for lung cancer. ${ }^{135}$ Association of exosomal ncRNAs and lung cancer can be explored to identify novel biomarkers for tumor targeted diagnosis and therapy. ${ }^{132}$ Numerous lines of publications have indicated that exosomes and exosomal ncRNAs exert important roles in multiple cellular and molecular processes linked to lung cancer, thus providing new diagnostic biomarkers and therapeutic targets in lung cancer.

EMT is tightly associated with tumor invasion and metastasis by promoting lung cancer cells infiltration and migration. ${ }^{136}$ Kim et al. reported that miR-23a is significantly enriched in TGF- $\beta 1$-treated human lung adenocarcinoma (LUAD) cells and is involved in EMT. ${ }^{137}$ Notably, bone marrow-derived mesenchymal stem cells (BMSCs) are essential components of cancer microenvironment and are involved in development of lung cancer. Zhang et al. demonstrated that BMSCs-derived exosomal miR-193a-3p, miR210-3p, and miR-5100 promote lung cancer cells invasion by activating signal transducer and activator of transcription 3 (STAT3) signaling pathway and triggering EMT. ${ }^{138}$ Furthermore, miR-499a-5p is upregulated in highly metastatic cells-derived exosomes and enhances cell proliferation, migration, and EMT by targeting the rapamycin (mTOR) pathway. ${ }^{139}$

Cellular proliferation and migration are implicated in cancer onset and development. ${ }^{140}$ It has been reported that exosomal miR-96 plays a role in lung cancer cells proliferation and migration by targeting LIM-domain only protein 7 (LMO7). ${ }^{141}$ Non-small cell lung cancer (NSCLC) accounts for approximately $85 \%$ of all lung cancer cases. ${ }^{142}$ Exosomes released from gemcitabine-resistant A549 cells can transfer miR-222-3P to target cells and promote cell proliferation, migration, and invasion by targeting suppressor of cytokine signaling 3 (SOCS3). ${ }^{143}$ On the contrary, EC-derived exosomes are characterized by high level of miR-126 and play a role in inhibiting cell proliferation and reducing loss of malignancy of NSCLC cells. ${ }^{144}$ Mechanistically, exosomal miR-126 suppress NSCLC development by targeting insulin receptor substrate 1 (IRS1) and vascular endothelial growth factor (VEGF). A549 NSCLC cells-derived exosomal miR-208a inhibits NSCLC cell proliferation by targeting p21 thus activating the AKT/mTOR pathway. ${ }^{145}$ In addition, exosomal miR-512 suppresses cell proliferation by targeting TEA domain family member 4 (TEAD4). ${ }^{146}$

Oxygen and nutrients are necessary for survival of cancer cells, thus angiogenesis is essential for tumor growth and metastasis. ${ }^{147}$ Zhuang et al. reported that exosomal miR-9 enhances angiogenesis by activating JAK/STAT pathway. ${ }^{148}$ Moreover, human bronchial epithelial (HBE) cells-derived exosomal miR-21 can promote angiogenesis by activating STAT3 and increasing expression of VEGF. ${ }^{149}$ Furthermore, circulating exosomal miR23a levels are positively correlated with lung cancer proangiogenic activities. Hypoxic lung cancer cells promote angiogenesis by repressing the tight junction protein ZO-1 through exosomal miR-23a. ${ }^{150}$ Exosomal miR-126 secreted by NSCLC cells can trigger angiogenesis and accelerate lung cancer progression. ${ }^{144}$ In addition, miR-210 packaged in exosomes from tumor cells stimulates angiogenesis. ${ }^{151}$

Metastasis is a primary feature of cancer. Bone metastasis is common in patients with lung cancer. ${ }^{131}$ MiR-21 in A549 cellsderived exosomes promotes tumorigenesis and osteoclastogenesis by targeting the PDCD4 pathway. ${ }^{152}$ In addition, immune responses are involved in cancer progression. Fabbri et al. demonstrated that NSCLC cells-derived exosomes are characterized by high expression levels of miR-21, miR-27b, and miR-29a. Notably, exosomal miR-21 and miR-29a can promote tumor growth and metastasis by targeting Toll-like receptor (TLR) and inducing pro-metastatic inflammatory responses. ${ }^{80}$ Moreover, overexpression of exosomal miR-192 significantly appeased osseous metastasis by suppressing secretion of proangiogenic factors. ${ }^{82}$

Exosomal IncRNAs have been reported to be highly correlated with lung cancer. Exosomal IncRNAs such as H19, MALAT1, HOTAIR, UCA1, Inc-MMP2-2, GAPLINC, TBILA, AGAP2-AS1, and SOX2-OT play several roles in pathological processes including cell proliferation, migration, invasion, and EMT linked to lung cancer. ${ }^{153} \mathrm{Li}$ et al. reported that GAS5 in exosomes is not only important for cancer development, but a promising biomarker for diagnosis patients with early NSCLC. ${ }^{154}$ Teng et al. found that exosomal SOX2-OT can be used as an effective noninvasive plasma-based tumor marker for lung squamous cell carcinoma (LSCC). ${ }^{155}$ 


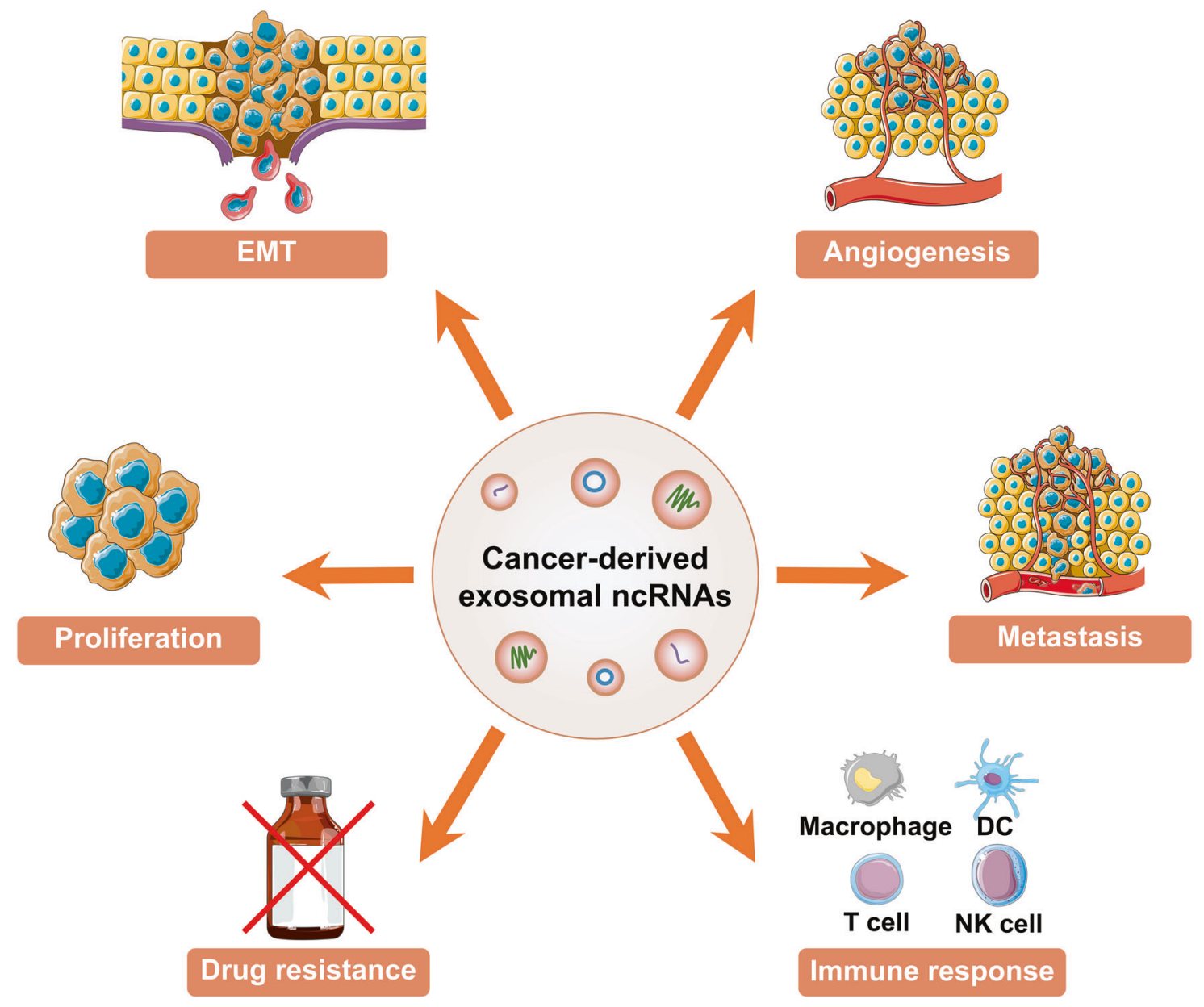

Fig. 5 The roles of exosomal ncRNAs in cancer. Exosomal ncRNAs play a role in cancers, including EMT, proliferation, angiogenesis, metastasis, drug resistance, and immune response. This figure was created with the aid of Servier Medical Art (https://smart.servier.com/). EMT epithelialmesenchymal transition, DC dendritic cell, NK natural killer

Exosomal circRNAs are involved in the development of lung cancer. CircSATB2 are enriched in NSCLC cells and can be transported to other cells by exosomes to facilitate cell proliferation, migration, and invasion of NSCLC cells, and trigger abnormal proliferation of bronchial epithelial cells. ${ }^{156}$ Exosomal has-circ0014235 promotes NSCLC progression by targeting miR-520a-5p/ cyclin-dependent kinase 4 (CDK4) axis. ${ }^{157}$ Besides, circRNA-002178 shuttled by exosomes can be transferred to $\mathrm{CD}^{+} \mathrm{T}$ cells thus promoting generation of programmed death-ligand 1 (PDL1)/ programmed cell death protein 1 (PD1) in LUAD. ${ }^{158}$ In addition, the expression levels of exosomal circ-0007761, circ-0047921, circ0056285, circ-0008928, circRNA-102481, circ-MEMO1, circ-ARHGAP10, circ-PIP5K1A, and circ-FARSA are changed in NSCLC, ${ }^{159-165}$ while that of exosomal circ-0000690, circ-0001346, circ-0001439, and circ-0001492 are significantly increased in LUAD, which have potential as promising diagnostic biomarkers. ${ }^{166}$

Exosomal ncRNAs in BC. BC is the most frequent female malignant tumor globally and $70-80 \%$ patients present with early, non-metastatic BC which is considered curable. ${ }^{167}$ Approximately 2.1 million cases of $B C$ were diagnosed in women and 626,679 breast cancer-related deaths were reported in $2018 .^{168}$ Over the past decades, increasing evidence has shown that exosomal ncRNAs are closely associated with BC development. ${ }^{169}$

Exosomal miRNAs play roles in cellular proliferation, migration, and invasion of BC. A previous study reported that exosomal miR$10 \mathrm{~b}$ is highly expressed in BC cells and promotes invasion by inhibiting expression of HOXD10 and KLF4. ${ }^{170}$ Exosomal miR-1246 is highly expressed in breast cancer cells and enhances cell proliferation and invasion by targeting CCNG2. ${ }^{171}$ On the contrary, exosomal miR-134 downregulates and inhibits cell proliferation, migration and invasion by targeting STAT5B in BC. ${ }^{172}$ Overexpression of miR-130a-3p in BC stem cells inhibits cellular proliferation, migration, and invasion by targeting RAB5B. ${ }^{173}$ In addition, miR-127, miR-197, miR-222, and miR-223 shuttled by exosomes inhibits cell proliferation by suppressing CXCL12.

Exosomal miRNAs are also involved in metastasis of BC. Cancerassociated fibroblasts (CAFs) are essential components of tumor microenvironment and play important roles in tumor development and metastasis. Exosomal miR-9 is highly expressed in breast CAFs and promotes switch of fibroblasts to CAF phenotype. ${ }^{174} \mathrm{Wu}$ et al. revealed that exosomal miR-16 and miR-148a from focal adhesion kinase knockout CAFs are upregulated and ameliorate tumor cell metastasis. ${ }^{175}$ CAFs-derived exosomal miR-21, miR-143, and miR-378e promote stemness and EMT phenotype of BC cells. ${ }^{176}$ Moreover, miR$1910-3 p$ shuttled by exosomes promotes cell proliferation and metastasis by targeting MTMR3 and NF-KB signaling pathway. ${ }^{104}$ In addition, exosomal miR-503-3p, miR-4269, miR-30e-3p, miR-105, miR122 , miR-200, miR-939, and miR-940 play crucial roles in promoting BC progression and metastasis. ${ }^{177-182}$

Apart from these, exosomal miRNAs participate in other cellular process of BC. For instance, BC cells-derived exosomal miR-20a-5p enhances differentiation of osteoclasts by targeting SRCIN1. ${ }^{183}$ MiR$23 \mathrm{~b}$ is upregulated in BC cells-derived exosomes and reduces expression of MARCKS, a key regulator of cell cycling and motility. ${ }^{184}$ Notably, miR-210 from BC cells-derived exosomes can promote 
angiogenesis. ${ }^{185}$ On the contrary, miR-16 and miR-100 in exosomes from mesenchymal stem cells (MSCs) reduce secretion of VEGF in tumor cells and inhibit angiogenesis. ${ }^{186,187}$

Exosomal IncRNAs play key roles in BC progression and are potential diagnostic biomarkers for BC. LncRNA MALAT1 shuttled by $\mathrm{BC}$ exosomes can promote cell proliferation. ${ }^{188}$ Exosomal IncRNA GS1600G8.5 are highly expressed in brain metastatic breast cancer cells and are implicated in destroying the BBB system and promoting transfer of cancer cells across the BBB. ${ }^{189}$ Moreover, expression of exosomal IncRNAs, including H19, SUMO1P3, XIST, and HOTAIR is upregulated in patients with $\mathrm{BC}$ indicating that they can serve as promising diagnostic biomarkers for BC. ${ }^{190-194}$

Exosomal circRNAs exhibit various roles in BC. Yang et al. found that serum exosomal circPSMA1 from BC is highly upregulated and promotes $\mathrm{BC}$ tumorigenesis, migration, and migration through miR637/Akt1/ß-catenin (cyclin D1) axis. ${ }^{195}$ Besides, circHIF1A (circ0032138) is highly expressed in hypoxic CAFs-derived exosomes and modulates stem cell properties of BC through miR-580-5p/CD44 axis. ${ }^{196}$ Besides, exosomal circHIF1A significantly promotes BC growth and metastasis by activating AKT/STAT3 signaling pathway and suppressing expression of P21. ${ }^{197}$ On the contrary, Wang et al. reported that expression of several exosomal circRNAs was downregulated in BC cells. ${ }^{198}$

Exosomal ncRNAs in HCC. HCC is a major cause of cancer-related deaths worldwide. ${ }^{199}$ Notably, HCC is commonly diagnosed in cirrhosis patients. ${ }^{200}$ Recent studies have explored the biological functions of exosomal ncRNAs in initiation and development of HCC and report that exosomal ncRNAs can be used as noninvasive biomarkers for HCC. ${ }^{201}$

Exosomal miRNAs are closely associated with the pathology of HCC. Cui et al. reported that exosomal miR-224 promotes cell proliferation by targeting glycine N-methyltransferase. ${ }^{202}$ In addition, exosomal miR-93 can stimulate proliferation and invasion of HCC by suppressing TIMP2/TP53INP1/CDKN1A. ${ }^{203}$ Moreover, HCC cell-derived exosomal miR-665 promotes tumor cell proliferation by targeting MAPK/ERK signal pathway. ${ }^{204}$ Studies report that serum exosomal miR-1247-3p is implicated in lung metastasis in HCC patients. Exosomal miR-1247-3p released from high-metastatic HCC cells promotes tumor development by activating the $\beta 1$-integrin-NF- $\mathrm{kB}$ signaling pathway and releasing pro-inflammatory cytokines, including IL-6 and IL-8. ${ }^{205}$ HCC cellderived exosomal miR-210 can be transferred into ECs and enhances angiogenesis by targeting SMAD4 and STAT6. ${ }^{206}$ Similarly, miR-155 shuttled by exosomes from hypoxia-treated HCC cells is involved in tube formation of ECs and tumor angiogenesis. ${ }^{207}$ In addition, overexpression of exosomal miRNAs, including miR-224, miR-21, miR-92b, miR-93, miR-10b-5p, hsamiRNA-1298, and miR-215-5p can be used as diagnostic markers for patients with HCC. ${ }^{202,203,208-212}$ On the contrary, levels of some exosomal miRNAs, including miR-9-3p, miR-125b, miR-638, miR718, miR-101, miR-106b, miR-122, miR-195, and miR-744 are downregulated in HCC patients. ${ }^{213-218}$

LncRNAs shuttled by exosomes play key roles in regulating tumor cell proliferation, angiogenesis, invasion, and metastasis. ${ }^{219}$ $\mathrm{Li}$ et al. revealed that IncRNA FAL1 is highly expressed in serum exosomes from HCC patients and promotes HCC cell proliferation and metastasis through competitively binding to miR-1236. ${ }^{220}$ $\mathrm{H} 19$ is upregulated in exosomes from propofol-treated HCC cells and stimulates tumor cell proliferation, migration, and invasion through the miR-520a-3p/LIMK1 axis. ${ }^{221}$ Moreover, exosomal IncRNA $\mathrm{H} 19$ is significantly upregulated in $\mathrm{CD}^{+} 0^{+}$liver cancer cells and is implicated in promoting angiogenesis and regulating tumor microenvironment. ${ }^{222}$ In addition, IncRNA TUC339 is highly expressed in exosomes from HCC cells and it stimulates M2 macrophage polarization, thus enhancing tumor cell migration, invasion, and EMT. ${ }^{223}$ Similarly, HCC-derived exosomal DLX6-AS1 triggers M2 macrophage polarization by targeting the miR-15a-
5p/CXCL17 axis. $^{71}$ LincRNA VLDLR encapsulated in extracellular vesicles was highly expressed in HCC cells and promotes cellular stress responses. ${ }^{24}$ Sorafenib-treated HCC cell-derived exosomal lincRNA ROR is upregulated and suppresses death of recipient HCC cells by targeting the p53 signaling pathway. ${ }^{225}$ Ma et al. reported that expression of exosomal ASMTL-AS1 was highly correlated with the stage, metastasis, and prognosis in HCC. ${ }^{226}$ High expression level of exosomal IncRNA-ATB is significantly correlated with lower overall survival in HCC patients. Therefore, exosomal IncRNA-ATB is a promising prognostic biomarker for HCC. $^{208}$ In addition, expression levels of LINC00161, LINC00635, IncRNA-RP11-583F2.2, Inc-FAM72D-3, Inc-EPC1-4, and IncRNA$\mathrm{HEIH}$ in exosomes are high in HCC patients. ${ }^{212,227-230}$ On the contrary, SENP3-EIF4A1 and linc-FAM138B are downregulated in plasma exosomes in HCC patients. Exosomal SENP3-EIF4A1 can be transferred into HCC cells thus inhibiting tumor cell growth, and attenuate invasion and migration of HCC cells, ${ }^{231}$ while exosomal linc-FAM138B plays a role in repressing HCC growth by targeting miR-765. ${ }^{232}$

Exosomal circRNAs play a role in cellular processes of HCC, such as cell proliferation, angiogenesis, metastasis, and EMT. Huang et al. claimed that exosomal circRNA-100338 secreted by highly metastatic HCC cells is significantly upregulated and can be transferred to human umbilical vein endothelial cells (HUVECs). Exosomal circRNA-100338 can stimulate cell proliferation, angiogenesis, and vasculogenic mimicry formation of HUVECs and promote tumor metastasis. ${ }^{233}$ In addition, exosomal circRNA Cdr1as is highly expressed in HCC cells and can be transferred to surrounding cells. Overexpression of Cdr1as stimulates cell proliferation and migration by targeting the miR-1270/AFP axis. ${ }^{234}$ Circ-ZNF652 is highly expressed in exosomes from HCC cells and in serum of HCC patients. Exosomal circ-ZNF652 is implicated in stimulating cell proliferation, migration, invasion, and glycolysis by targeting miR-29a-3p/GUCD1 axis. ${ }^{235}$ Moreover, expression of circFBLIM1 is upregulated in HCC serum exosomes and promotes tumorigenesis and glycolysis by targeting miR-338. ${ }^{236}$ Exosomal circ-0004277 plays a role in inducing malignant phenotype of HCC by suppressing expression of ZO-1 and increasing tumor cell progression, migration, and EMT. ${ }^{237}$ In addition, the level of serum exosomal CircPTGR1 is upregulated in HCC patients and plays a role in facilitating tumor metastasis by targeting miR449a/MET pathway. ${ }^{238}$ Overexpression of exosomal has-circ-0039411 increases secretion of matrix metallopeptidase 2 (MMP2) by sponging miR-136-5p. High expression levels of exosomal has-circ0039411 and MMP2 are correlated with tumor metastasis and low overall survival of HCC patients. ${ }^{239}$ HCC cell-derived exosomes exhibit a high expression level of circUHRF1. Exosomal circUHRF1 can trigger dysfunction of natural killer (NK) cells thus inducing immunosuppression in HCC patients. ${ }^{240}$ Expressions of exosomal circRNAs, such as circAKT3, has-circ-0004001, has-circ-0004123, has-circ-0075792, circ-0061395, and circTMEM45A are also highly upregulated in HCC patients. ${ }^{241-243}$ However, exosomal circ0051443 is downregulated in plasma exosomes from HCC patients, which exerts a role in inducing cell apoptosis and inhibiting malignant behaviors. ${ }^{244}$ Wang et al. reported that level of exosomal has-circ-0074854 is also downregulated in HCC patients and plays a role in inhibiting tumor cell migration and invasion by repressing M2 macrophage polarization. ${ }^{245}$

Exosomal nCRNAs in CRC. CRC is a common cause of cancerassociated deaths worldwide. Approximately 1.2 million CRC cases are diagnosed and 600,000 people die with CRC every year. ${ }^{246}$ Previous studies report that exosomal ncRNAs play important roles in CRC.

Exosomal miRNAs exert vital effects on different progression of CRC. $^{247}$ First, exosomal miR-183-5p promotes tumor development and induces cell proliferation, invasion, and tube formation of ECs. ${ }^{248}$ Second, exosomal miR-25-3p and miRNA-146a-5 are 
corelated with angiogenesis and tumorigenesis of CRC, respectively. ${ }^{249,250}$ Third, high expressed miR-17-5p in exosomes promotes CRC metastasis. ${ }^{251}$ Fourth, exosomal miR-210 plays a role in promoting EMT and anoikis resistance. ${ }^{252}$

Besides, several exosomal miRNAs modulate the metastasis of CRC. CRC-derived exosomal miR-934 is involved in inducing liver metastasis of CRC by mediating cellular communication between tumor-associated macrophages and CRC cells. ${ }^{253}$ In addition, miR$1255 b-5 p$ is highly expressed in hypoxic-treated exosomes from mouse CRC. Exosomal miR-1255b-5p inhibits EMT, CRC development, and liver metastasis by regulating expression of human telomerase reverse transcriptase (hTERT). ${ }^{254}$ Furthermore, highmetastatic CRC-derived exosomal miR-106b-3p induces tumor cell migration, invasion, EMT, and lung metastasis by targeting deleted in liver cancer-1 (DLC-1). ${ }^{255}$

Numerous studies have found that expression levels of serum exosomal miRNAs, including let-7a, miR-1229, miR-1246, miR-150, miR-21, miR-223, miR-23a, miR-301a, miR-17-5p, miR-92a-3p, miR$6803-5 p$, and miR-320d are significantly upregulated in primary CRC patients. ${ }^{256-260}$ In addition, some plasma exosomal miRNAs, such as miR-27a and miR-130a are upregulated in CRC and can serve as noninvasive biomarkers for CRC. ${ }^{261}$ On the contrary, low expression levels of exosomal miRNAs, including miR-874, miR$30 a-5 p$, and miR-128-3p are highly correlated with tumor metastasis, differentiation, and advanced TNM stage. ${ }^{262-264}$

Moreover, exosomal IncRNAs play important roles in CRC. Exosomal RPPH1, MALAT1, NNT-AS1 promotes tumor progression, including cell proliferation, invasion, migration, and metasta$\mathrm{sis}^{265,266}$ while exosomal LINC02418 and H19 induces tumorigenesis and development. ${ }^{267,268}$ Exosomal HOTTIP is highly expressed in mitomycin-resistant CRC cells and promotes mitomycin resistance. ${ }^{269}$ Notably, low levels of exosomal HOTTIP are highly correlated with poor overall survival of CRC patients. ${ }^{270}$ Besides, studies report that exosomal IncRNAs, including GAS5, CRNDE-h, CRNDE-p, LINC02418, CCAT2, LNCV6-116109, LNCV6-98390, LNCV6-38772， LNCV-108266， LNCV6-84003， LNCV6-98602, FOXD2-AS1, NRIR, and XLOC-009459 are high expressed in CRC patients. ${ }^{267,271-276}$ In addition, serum exosomal IncRNA UCA1 is downregulated in CRC patients. ${ }^{277}$

Recent studies reported that exosomal circRNAs are involved in pathophysiology of CRC. Shang et al. determined that exosomeencapsulated circPACRGL from CRC patients stimulates tumor cell proliferation, migration, invasion, and metastasis, as well as neutrophil differentiation. ${ }^{278}$ Moreover, exosomal circFMN2 mediates cell proliferation and migration, ${ }^{279}$ while exosomal circlFT80 is implicated in promoting CRC development. ${ }^{280}$ Exosomal hascirc- 0005963 and circ- 133 regulate the process of CRC by targeting miR-122/M2 isoform of pyruvate kinase (PKM2) axis ${ }^{281}$ and miR133a/GEF-H1/RhoA axis, ${ }^{282}$ respectively. Circulating exosomal hsacirc-0004771 is significantly upregulated in CRC patients and is a novel potential diagnostic biomarker of CRC. ${ }^{283}$

Exosomal ncRNAs in GC. GC is a common cause of cancer-related death worldwide. In 2018, approximately 784,000 GC-related deaths were reported. ${ }^{284}$ Emerging evidence suggested that exosomal ncRNAs are involved in development of GC.

Cellular proliferation, migration, and invasion are closely associated with GC. Exosomal miR-1290 is upregulated in GC patients and stimulates proliferation, migration, and invasion of GC cells by downregulating expression of naked cuticle homolog 1 (NKD1). ${ }^{285}$ GC tissue-derived mesenchymal stem cells (GCMSCS)-derived exosomal miR-221 play a role in tumor cell growth and migration. ${ }^{286}$ Exosomal miR-301a-3p promotes progression and metastasis of GC cells by regulating PHD3/HIF-1a. ${ }^{287}$ Exosomal miR-15b-3p promotes tumor cell proliferation, migration, and invasion by targeting DYNLT1/Caspase-3/Caspase- 9 pathway. ${ }^{288}$ In addition, miR-34 packaged in exosomes from gastric CAFs shows low expression levels. Notably, overexpression of exosomal miRNA-34 can inhibit tumor cell proliferation, invasion, and motility. ${ }^{289}$

Besides, exosomal miRNAs take part in the progression of metastasis and angiogenesis of GC. MiR-let-7 is highly expressed in metastatic GC cell-derived exosomes. Exosomal miR-let-7 promotes tumorigenesis and metastasis by targeting RAS and HMGA2. ${ }^{290}$ Exosomal miR-21-5p promotes GC peritoneal metastasis by stimulating mesothelial-to-mesenchymal transition of peritoneal mesothelial cells (PMCs). Mechanistically, exosomal miR-21-5p induces metastasis by targeting SMAD7 and activating TGF- $\beta /$ Smad signaling pathway. ${ }^{291}$ Wang et al. reported that exosomes released from GC cells exhibit a high level of miR-27a. Exosomal miR-27a induces CAFs and promotes tumor cell motility and metastasis by targeting CSRP2. ${ }^{292}$ Gastric CAFs-derived exosomal miR-139 suppresses gastric cancer metastasis and development by reducing matrix metalloproteinase 11 (MMP11). ${ }^{293}$ GC cell-derived exosomal miR-130a can be delivered into ECs to induce angiogenesis and tumor growth by regulating c-MYB. ${ }^{294}$ Studies report that exosomal miRNAs, such as miR-19b3p, miR-106a-5p, miR-1246, miR-107, miR-196a-1, miR-106a, and miR-155-5p are all upregulated in GC patients. ${ }^{295-300}$

Exosomal IncRNAs regulate the progression of GC through diverse mechanisms. Overexpression of exosomal HOTAIR induces proliferation, migration, and invasion of GC cells by increasing KRAS. $^{301}$ Exosomal LINC01559 promotes tumor progression by regulating miR-1343-3p/ phosphoglycerate kinase 1 (PGK1) and activating PI3K/AKT pathway. ${ }^{302}$ GC cells-derived exosomal IncRNA HEIH can be delivered into normal gastric cells and promote malignant transformation of GC by promoting expression of EZH2. ${ }^{303}$ Wang et al. reported that exosomal HOTTIP is implicated in cisplatin resistance in GC patients by targeting miR218/HMGA1 axis. $^{304}$ Numerous lines of evidence suggested that exosomal IncRNAs, such as UEGC1, HOTTIP, GC1, MIAT, H19, IncSLC2A12-10:1, CEBPA-AS1, ZFAS1 are highly expressed. ${ }^{118,305-311}$ On the contrary, exosomal IncRNAs, such as GNAQ-6:1 and PCSK22:1 show low expression levels in GC patients. ${ }^{312,313}$

Exosome-mediated circRNAs play important roles in GC. Xie et al. demonstrated that circSHKBP1 is highly expressed in exosomes from GC patients and the level of exosomal circSHKBP1 was reduced after gastrectomy. Exosomal circSHKBP1 promotes proliferation, migration, invasion, and angiogenesis of GC cells by targeting miR-582-3p/HUR/VEGF axis and through inhibition of HSP90 degradation. ${ }^{314}$ CiRS-133 encapsulated in exosomes secreted by GC cells can be transferred to preadipocytes. Exosomal ciRS-133 stimulates preadipocytes differentiation into brown-like cells by regulating miR-133/ PRDM16 pathway. ${ }^{315}$ Exosomal circNEK9 promotes proliferation, migration, invasion, and motility of recipient GC cells by modulating miR-409-3p/MAP7 axis. ${ }^{316}$ Moreover, circ-PVT1 is highly expressed in cisplatinresistant GC cells-derived exosomes. Exosomal circ-PVT1 promotes cisplatin resistance in GC cells by inducing cell autophagy and invasion and by inhibiting apoptosis. Overexpression of exosomal circ-PVT1 induces low expression of miR-30a-5p and high expression of YAP1 in GC cells. ${ }^{106}$ Circ29 packaged in exosomes can be transferred from GC cells to ECs and promote proliferation, migration, and tube formation of ECs by targeting the miR-29a/ VEGF pathway. ${ }^{317}$ In addition, circNHSL1 is highly expressed in exosomes released from GC cells. Exosomal circNHSL1 promotes migration, invasion, and glutaminolysis of GC cells by targeting miR-149-5p/YWHAZ axis. ${ }^{318}$ Overexpression of exosomal circ0032821 from oxaliplatin-resistant GC cells promotes proliferation, migration, and invasion of GC cells by modulating miR-515-5p/ SOX9 pathway. ${ }^{319}$ Notably, exosomal has-circ-0065149 is downregulated in GC patients. ${ }^{320}$

Exosomal miRNAs in PCa. PCa is a heterogeneous disease. ${ }^{321}$ Approximately 160,000 PCa cases are diagnosed each year in the United States. ${ }^{322}$ Accumulating studies indicate that exosomes are 
Li et al.

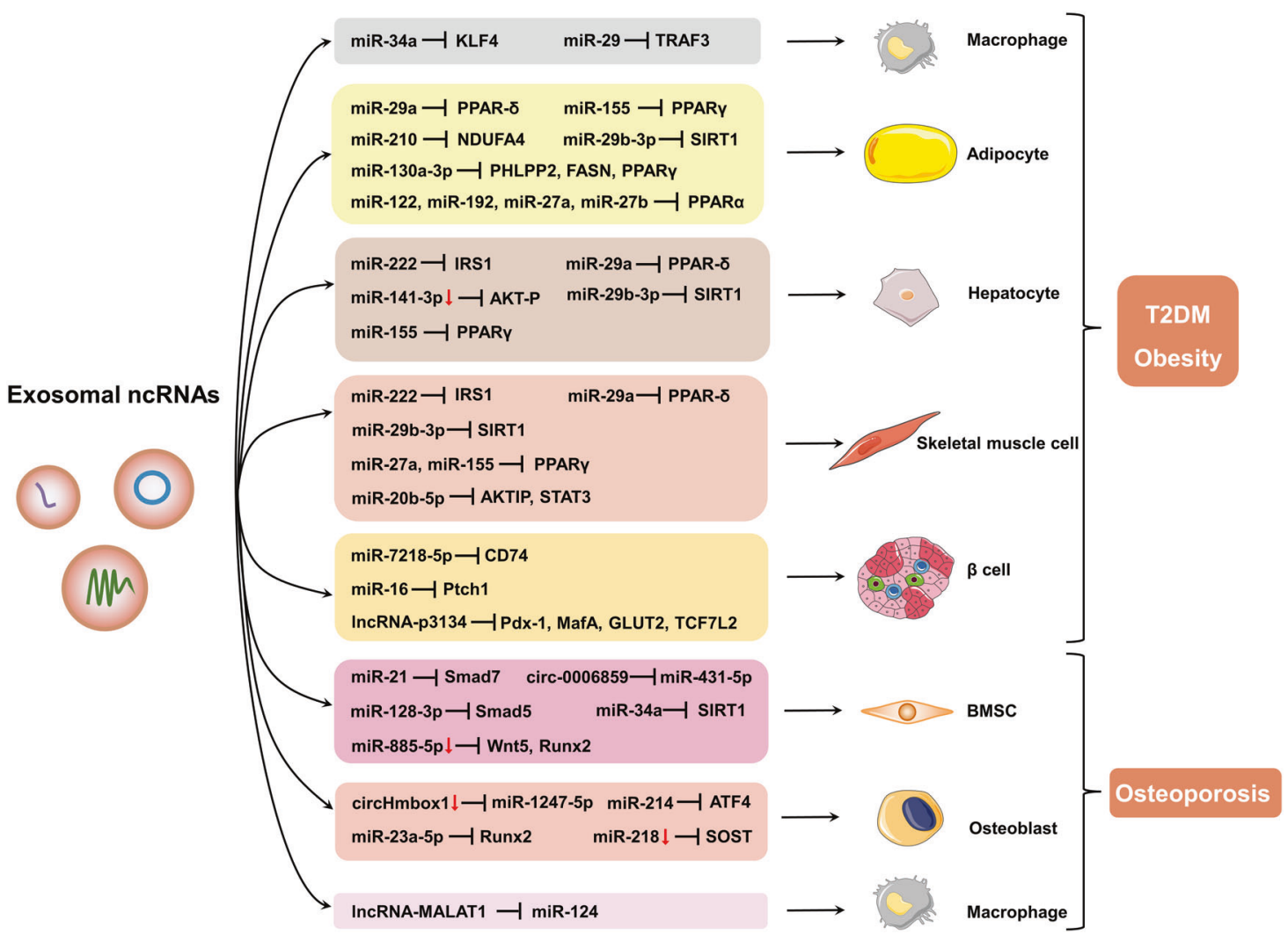

Fig. 6 The role of exosomal ncRNA in the pathological process of metabolic diseases. Exosomes secreted by different tissues can be released into the circulation and transported to other organs, where they are internalized by recipient cells, mediating metabolic regulation. This figure was created with the aid of Servier Medical Art (https://smart.servier.com/)

implicated in PCa tumor development. Dysregulated exosomal ncRNAs are involved in tumor initiation and progression of $\mathrm{PCa}{ }^{323}$

Exosomal miRNAs are implicated in PCa. MiR-217 encapsulated in exosomes exerts roles in promoting tumor cell proliferation, invasion, and EMT. ${ }^{324}$ MiR-1246, a tumor inhibitor, was downregulated in $\mathrm{PCa}$ cell-derived exosomes. Overexpression of exosomal miR-1246 inhibits tumor cell proliferation, migration, and invasion and promoted cell apoptosis by suppressing EMT. Exosomal miR-205 released from human bone marrow mesenchymal stem cells (hBMSCs) promotes tumor cell apoptosis and inhibits proliferation, migration, and invasion of PCa cells by targeting RHPN2. ${ }^{325}$ Exosomal miR-26a exerts a vital role in mediating tumor growth and tumor cell metastasis. ${ }^{326}$ Tumorassociated macrophages enhances $\mathrm{PCa}$ progression through exosomal miR-95. ${ }^{327}$ MiR-183 encapsulated in exosomes is upregulated in PCa patients and promotes proliferation, migration, and invasion of tumor cell by downregulating expression of TPM $1 .{ }^{328}$ Ye et al. reported that exosomal miR-141-3p promotes osteoblastic metastasis of PCa by modulating activity of osteoblasts. ${ }^{329}$ Huang et al. suggested that overexpression of plasma exosomal miR-1290 and -375 was significantly correlated with poor overall survival of PCa. ${ }^{330}$ Plasma exosomal miRNAs such as miR-1285, miR-622, miR-221, miR-145, and serum exosomal miRNAs such as miR-1274a, miR-1207-5p, miR-885-5p, miR-874, miR-766, miR-640, miR-636, miR-486-5p, miR-375, miR-346, and miR-141 are significantly upregulated in $\mathrm{PCa}$ patients and are promising biomarkers for diagnosis of $\mathrm{PCa}{ }^{331,332}$

Exosomal IncRNAs are implicated in diverse molecular processes involved in PCa progression. LncRNA MYU shuttled by exosomes is highly expressed in PCa patients and can be delivered into adjacent cells. Notably, exosomal IncRNA MYU induces cell proliferation and migration by competitively binding miR-184 and promoting expression of c-Myc. ${ }^{333}$ PCa-derived exosomal
SChLAP1 is highly expressed and significantly correlated with the level of prostate specific antigen (PSA) and tumor cell invasion. ${ }^{334}$ Ozgur et al. demonstrated that exosomal $\mathrm{H} 19$ is involved in PCa by regulating androgen receptor pathway. ${ }^{335}$

CircRNAs highly expressed in exosomes can be transferred from tissues into various body fluids and are potential diagnostic biomarkers for PCa. ${ }^{336}$ Has-circ-0044516 shuttled by exosomes induces tumor cell metastasis and inhibits tumor cell apoptosis by sponging miR-29a-3p. ${ }^{337}$ Exosomal circ-XIAP is upregulated in docetaxel-resistance $\mathrm{PCa}$ cells and promotes docetaxel resistance by targeting miR-1182/TPD52 axis. $^{338}$

In general, exosomal ncRNAs are involved in pathological cellular processes including cell proliferation, migration, invasion, metastasis, angiogenesis, and EMT associated with diverse cancers. Accumulating evidence over the last decade has further revealed that exosomal ncRNAs can participate in multiple processes contributing to cancer development, diagnosis biomarkers, and therapeutic effects, showing the dual characteristics of promoting and suppressing cancer. In this part, we mainly discuss the roles of exosomal miRNAs, IncRNAs, and circRNAs in lung cancer, BC, HCC, CRC, GC, and PCa. Notably, studies have explored roles of exosomal ncRNAs in other cancers, including esophageal cancer, pancreatic cancer, ovarian cancer, and leukemia. ${ }^{339}$

The roles of exosomal ncRNAs in metabolic diseases Previous studies report that exosomal ncRNAs play important roles in remote tissues. NcRNAs-encapsulated exosomes are implicated in various processes that involved in development of metabolic diseases, such as T2DM, obesity, and osteoporosis (Fig. 6).

Exosomal ncRNAs in T2DM. T2DM is a prevalent chronic disease that causes cardiovascular, renal, retinal, and neurological complications, and is a major cause of death and disability 
worldwide. $^{340,341}$ T2DM is the most common clinical type of diabetes accounting for over $90 \%$ of diabetes cases, which is characterized by relative insulin deficiency as a result of progressive inadequate insulin secretion and varying degrees of IR in peripheral tissues, such as adipose tissues, skeletal muscle, and liver tissues. ${ }^{9,342}$ Aging, obesity, and other cardiovascular risk factors promote the development of T2DM. ${ }^{340}$ However, the roles of these factors in promoting the pathological process in T2DM has not been fully elucidated. Recent studies on exosomes and their ncRNA cargoes have been conducted to explore the underlying mechanisms.

MiRNAs are the most explored ncRNAs, and several exosomal miRNAs are closely linked to T2DM. Adipose tissue is an endocrine organ that functionally regulates systematic energy homeostasis by releasing various endocrine factors which modulate glucose and lipid metabolism. ${ }^{9,343}$ In addition, adipose tissue is a major source of circulating exosomal miRNAs, which modulate glucose tolerance and the expression of fibroblast growth factor 21 (FGF21) in the liver. ${ }^{344}$ MiRNAs profiles in adipocyte-derived exosomes vary under different physiopathological conditions of adipose tissue. ${ }^{345}$ Adipocyte-derived exosomal miRNAs, such as miR-34a, ${ }^{346}$ miR-222, ${ }^{347}$ miR-27a, ${ }^{348}$ and miR-802-5p ${ }^{85}$ can promote IR via regulating Krüppel-like factor 4 (KLF4), insulin receptor substrate 1 (IRS1), peroxisome proliferator-activated receptor $Y$ (PPARY), and HSP60. Hepatocyte uptake of exosomes from adipocytes in obese mice containing less miR-141-3p than that in healthy mice, and the reduced absorption of miR-141-3p resulted in reduced glucose uptake in hepatocytes. ${ }^{349} \mathrm{~A}$ recent study reported that exosomal miR-130b-3p from adipocytes isolated from epididymal fat of HFD mice or incubated with high glucose/high lipid level aggravates myocardial ischemia/reperfusion injury in nondiabetic mice, by inhibiting various antiapoptotic and cardioprotective signaling, mainly AMP-activated protein kinase (AMPK). ${ }^{350}$ In addition to adipocytes, macrophages can release exosomes containing miRNAs that regulate glucose metabolism. Adipose tissue macrophage (ATM)-derived exosomal miR-155 in obese mice promotes glucose intolerance and IR by targeting PPAR ${ }^{351}$ Similarly, ATM-derived exosomal miR-29a can be transported into adipocytes, myocytes, and hepatocytes, thus inducing IR in vitro and in vivo through PPAR- $\delta .^{352}$ MiR-690 in exosomes derived from M2-polarized macrophages can alleviate glucose tolerance and IR in obese mice by targeting Nadk, a gene encoding $\mathrm{NAD}^{+}$kinase. $^{353}$ Exosomal miR-210 released from high glucose (HG)-induced ATM promotes the development of diabetic obesity in mice by inhibiting NADH dehydrogenase ubiquinone 1 alpha subcomplex 4 (NDUFA4) in adipocytes, leading to reduced glucose uptake and mitochondrial complex IV (CIV) activity. ${ }^{354}$ Exosomal miR-21-5p derived from HG-stimulated macrophages induces inflammation, ROS production, and podocyte injury in diabetic nephropathy (DN) mice by modulating A20. ${ }^{79}$

Liver-derived exosomal miR-130a-3p ameliorates glucose intolerance and IR by repressing pleckstrin homology domain leucinerich repeat protein phosphatases 2 (PHLPP2) thus triggering AKT/ Akt substrate of the $160 \mathrm{kDa}$ (AS160)/glucose transporter 4 (GLUT4) axis in adipocytes. ${ }^{355}$ Expression level of hepatocytereleased exosomal miR-7218-5p in HFD mice is lower compared with that in normal diet mice. MiR-7218-5p mimic transfection decreases islet $\beta$ cells proliferation by regulating CD74. ${ }^{356}$

Furthermore, skeletal muscle-derived exosomal miRNAs play important roles in T2DM, although only a few studies have explored these roles. Jalabert and colleagues found that exosomes extracted from quadriceps muscle in mice fed with high palmitate diet promoted proliferation of $\beta$ cells and isolated islets. MiR-16 is upregulated in these exosomes and can modulate Ptch1 associated with pancreas development. ${ }^{357}$

It has been reported that islet $\beta$ cells can secretes different miRNAs. When encapsulated by exosomes, the miRNAs can be transported to functionally act on different recipient cells. ${ }^{358}$
Specifically, it has been shown that $\beta$ cell-derived exosomal miR$29 \mathrm{~s}$ attenuate hepatic insulin sensitivity and regulate glucose homeostasis as a result of high levels of free fatty acids. ${ }^{359}$ Another study shows that exosomal miR-29 induces recruitment and activation of monocytes and macrophages as well as consequent inflammation in HFD mice. This promotes progression of diabetes by decreasing tumor receptor-associated factor 3 (TRAF3). ${ }^{42}$ The abundance of miR-26a is decreased in serum exosomes of overweight human and obese mice and is negatively correlated with clinical characteristics of T2DM. Several experiments have proven that exosomal miR-26a from $\beta$ cells can ameliorate peripheral IR induced by obesity. Moreover, upregulation of miR$26 a$ in $\beta$ cells reduces glucose-stimulated insulin secretion (GSIS) by inhibiting actin cytoskeleton remodeling and thus prevents obesity-induced islet hyperplasia. ${ }^{360}$ Exosomal miR-15a released from pancreatic $\beta$-cells can travel through the circulation and be absorbed by Müller cells. This triggers oxidative stress and causes retinal injury and apoptotic cell death under T2DM conditions. ${ }^{361}$ Additionally, $\beta$ cell-derived exosomal miR-127 enhances the migration and ability of islet ECs to form tubes. ${ }^{362}$

Bone marrow-derived MSC (BMSC)-derived exosomal miR-29b$3 p$ in aged mice can be absorbed by adipocytes, myocytes, and hepatocytes, which subsequently decreases insulin sensitivity both in vivo and in vitro via targeting SIRT1. Intriguingly, downregulation of nanocomplex-mediated miR-29b-3p in exosomes from BMSCs attenuates IR in aged mice. ${ }^{40}$

Circulating exosomal miR-20b-5p attenuates insulin-stimulated glycogen accumulation through regulating AKT-interacting protein (AKTIP) and STAT3. ${ }^{363}$ MiRNA profiles in plasma exosomes were altered in obese mice, including increased miR-122, miR-192, miR-27a-3p, and miR-27b-3p. By targeting PPARa, the treatment of lean mice with exosomes containing obesity-related miRNAs induces glucose intolerance and IR. ${ }^{78}$ Additionally, altered miRNAs encapsulated in circulating exosomes correlates with the adiponectin pathway in T2DM patients. ${ }^{364}$

In addition to miRNAs, exosomal IncRNAs are also emerging as significant factors in T2DM. Expression of serum exosomal IncRNAp3134 was elevated in T2DM patients. ${ }^{365}$ This IncRNA has been revealed to be interrelated with levels of fasting blood glucose and homeostasis model assessment $\beta$-cell function (HOMA- $\beta$ ). Overexpression of IncRNA-p3134 can promote GSIS and reduce apoptosis in $\beta$ cells, which provides a novel mechanism of glucose homeostasis regulation by IncRNAs. ${ }^{365}$ The circulating level of exosomal IncRNA-MALAT1 significantly decreases in patients with T2DM. $^{366}$

Exosomal IncRNAs also exert protective effect. Li and coworkers have shown that exosomal IncRNA H19 from MSCs can induce fibroblast proliferation and migration, as well as inhibit apoptosis and inflammation via abrogating miR-152-3p-mediated PTEN repression and hence facilitates diabetic wound healing. ${ }^{367}$ Exosomes-mimetic nanovesicles carrying a high level of $\mathrm{H} 19$ can serve as a nano-drug delivery system to neutralize the inhibitory effect of hyperglycemia on regeneration and speed up chronic wound healing. ${ }^{368}$ MSC-released exosomal IncRNA SNHG7 inhibits HG-stimulated endothelial-mesenchymal transition and tube formation of human retinal microvascular ECs by targeting miR$34 a-5 p / X B P 1$ axis. This provides a promising therapeutic method for diabetic retinopathy. ${ }^{369}$

Emerging evidence has unveiled the important role of exosomal circRNAs in T2DM complications. Our recent study has revealed that HG-stimulated ECs release exosomes containing circRNA0077930 to vascular smooth muscle cells (VSMCs) causing VSMC senescence, ${ }^{370}$ which may lead to diabetic vascular complications. Further, circRNAs in serum exosomes were different among patients with diabetic foot ulcer (DFU), non-DFU diabetes, and healthy cases. ${ }^{371}$ Among these modified circRNAs, the abundance of exosomal has-circ-0000907 and has-circ-0057362 were significantly increased in early DFU. Further experiments showed their 
12

role as promising biomarkers in the early diagnosis of DFU. ${ }^{371}$ It has been reported that circRNA cPWWP2A can be transported from retinal pericytes to ECs by exosomes in a paracrine manner. Further, high expression of CPWWP2A attenuates diabetesinduced retinal vascular dysfunction in vivo. ${ }^{372}$ Besides, a recent study has verified that serum exosomal circRNA DLGAP4 was elevated in patients with DN and rat models as compared with T2DM individuals without DN and normal rats. Furthermore, exosomal circRNA DLGAP4 have been shown to induce mesangial cell proliferation and fibrosis, as well as exacerbating $\mathrm{DN}$ in vivo by sponging miR-143 to motivate Erb-b2 receptor tyrosine kinase 3 (ERBB3)/NF-kB/matrix metalloproteinase-2 (MMP-2) axis. ${ }^{373}$

Collectively, exosomal miRNAs from a variety of sources, including adipose tissue, liver, skeletal muscle, islet $\beta$ cells, BMSC, are all involved in the pathological process of T2DM. Exosomal IncRNAs not only act as modulators (IncRNA-p3134 and MALAT1), but also have therapeutic effects on T2DM and its complications (H19 and SNHG7). Exosomal circRNAs mainly play vital roles in T2DM complications, such as DFU, DN, and retinal vascular disorders.

Exosomal ncRNAs in obesity. Obesity is defined as abnormal or excessive accumulation of body fat that presents a health risk to an individual. According to World Health Organization, obesity is diagnosed with a body mass index (BMI) greater than $30 \mathrm{~kg} / \mathrm{m} .{ }^{2,374}$ Obesity has grown into an epidemic with approximately 604 million adults affected worldwide in $2015 .{ }^{375}$ This disorder has caused enormous social burden due to multiple comorbidities such as diabetes, hyperlipidemia, cardiovascular diseases, and diverse cancers. ${ }^{376}$ Understanding the pathogenesis of obesity contributes to effective management of this harmful disorder.

Emerging evidence reveals that exosomes, as a mediator that intercellularly transports ncRNAs, play a pivotal role in the development of obesity and associated metabolic disorders. Santamaria-Martos and coworkers detected several plasma exosomal miRNAs candidates associated with BMI (e.g., let-7b, miR-146a), dyslipidemia (miR-29c) and fasting insulin (e.g., miR$222 / 223$, miR-26b) in obese and non-obese women. ${ }^{377}$ Moreover, it has been reported that exosomal IncRNA-H19 expression is correlated with waist circumference. ${ }^{366}$ Elsewhere, exosomal miR181b-5p and miR219-5p of immune cell origin that are induced by elafin (an anti-inflammatory protein) promote leptin expression in adipocytes to reduce food consumption, obesity, and hyperglycemia in HFD male mice. ${ }^{378}$ It has been reported that aerobic exercise regulates serum exosomal miRNAs in obese mice. It decreases the levels of miR-122, miR-192, and miR-22, which are associated with improved adipogenesis, insulin sensitivity, and hepatic steatosis. ${ }^{379}$ Similarly, exosomal miRNAs profiles are modified in obese patients after bariatric surgery. ${ }^{380,381}$

Obesity is recognized as a state with chronic low-grade inflammation. ${ }^{59}$ Adipose tissue is an active endocrine organ that releases a variety of cytokines, such as adiponectin, interleukins, and TNF-a. It also secretes other pro-inflammatory biomolecules including miRNAs, which leads to metabolic dysfunction, particularly IR. ${ }^{126}$ MiRNAs contained in exosomes have been identified as important mediators of the inflammation caused by obesity. Some exosomal miRNAs are identified to be differentially expressed in visceral adipose of obese subjects compared to lean subjects. Besides, these miRNAs might target genes associated with obesity-induced inflammation, such as transforming growth factor- $\beta$ (TGF- $\beta$ ) and Wingless and int-1 (Wnt)/ $\beta$-catenin signaling. ${ }^{382,383}$ Moreover, circulating exosomal miRNAs from obese mice have also been found to trigger adipose tissue inflammation in lean mice. ${ }^{78}$ Elsewhere, exosomal miR-34a from obese mice have been found to promote inflammation by regulating the M1to-M2 macrophage ratio. ${ }^{346}$ Additionally, exosomal miR-690 from M2-polarized macrophages regulates inflammation in obese mice. 353
There is direct or indirect association of obesity with IR disorder. $^{384}$ It has been reported that exosomal miRNAs profiles are modified by dysregulation of glucose metabolism in obese patients. $^{385}$ On the other hand, obesity can contribute to IR through transporting exosomal miRNAs to target cells and tissues, which participates in a variety of pathophysiological processes including inflammation response and insulin signaling pathway. ${ }^{76,384}$ As previously stated, exosomal miRNAs from adipose tissue, particularly miR-155, miR-34a, miR-222, miR-27a, miR-29a, miR-210, and miR-141-3p, are crucial regulators in the physiopathology of obesity-induced IR. ${ }^{346-349,351,352,354}$ In addition, miR192, miR-122, miR-27a-3p, and miR-27b-3p are upregulated in plasma exosomes in obese mice. Injection of exosomes transfected with these increased miRNAs contributes to central obesity in lean mice by targeting PPARa. ${ }^{78}$ On the contrary, miR-26a from $\beta$ cells and miR-690 from M2-polarized macrophages which are contained in exosomes can ameliorate IR induced by obesity. ${ }^{353,360}$

In summary, exosomal ncRNAs participate in regulation of obesity and are related to BMI, dyslipidemia, and waist circumference. In addition, they are considered to be crucial mediators of inflammation caused by obesity and IR disorder.

Exosomal ncRNAs in osteoporosis. Osteoporosis is characterized by loss of bone mass, degradation of bone microstructure. The disease contributes to increased bone fragility and risk of fracture. ${ }^{386,387}$ As the global population ages, the incidence of osteoporotic fractures is increasing with consequent enormous economic costs, reduced quality of life and lifespan. ${ }^{38,389}$ The maintenance of bone mass relies on the strictly coordinated balance between bone formation and bone resorption, and the main cells involved are osteoblasts and osteoclasts. Osteoporosis is accompanied by an increase in bone resorption and a decrease in bone formation. Recent studies have demonstrated that some exosomal ncRNAs play a pivotal part in the modulation of osteogenesis and bone resorption. ${ }^{390,391}$

Exosomal miRNAs derived from MSCs exert a potential regulatory effect on osteogenesis. ${ }^{392}$ During osteogenic differentiation in human BMSCs, the miRNAs profile contained in exosomes is changed during osteogenic differentiation in human BMSCs. Among the effected changes, miR-885-5p is expression of miR-885-5p is lowered and proven to serve as a negative regulator of BMSCs osteogenic differentiation by suppressing Wnt5 and runt-related transcription factor 2 (Runx2). ${ }^{393}$ According to Jiang et al., miR-21-bearing exosomes in osteoporosis patients repress osteogenesis by targeting small mothers against decapentaplegic homolog 7 (Smad7). ${ }^{394}$ Further, a study by Xu et al. reported that exosomal miR-128-3p derived from MSCs in aged rats inhibits osteogenesis and fracture healing through dampening Smad5. ${ }^{390}$

Emerging evidence indicates that osteoclast-derived exosomes can transfer miRNAs to osteoblast that regulate bone formation. Elsewhere, Sun et al. revealed that osteoclast-derived exosomes shuttling miR-214 represses the osteoblasts activity. ${ }^{395}$ Further, Li et al. demonstrated that exosomal miR-214-3p could reduce osteoblast activity in vitro and impair bone formation in vivo. On the contrary, inhibition of miR-214-3p in osteoclast was reported to facilitate bone formation in aging ovariectomized mice. ${ }^{396}$ Moreover, Yang and colleagues showed that osteoclast-released exosomes enriched with miR-23a-5p inhibits osteogenic differentiation via repressing Runx2. ${ }^{397}$

Exosomes can mediate muscle-bone crosstalk through transporting their miRNAs cargoes. Fulzele et al. proved that circulating muscle-derived exosomal miR-34a increases with age in mice. High expression of miR-34a in the exosomes from myoblasts impaired BMSC viability and promoted cellular senescence, as well as decreased SIRT1 expression in BMSCs. ${ }^{398}$ Other studies have also reported that Sirt1 promotes differentiation of osteoblast. $^{399,400}$ These findings suggests that there is a potential 
pattern of inter-organ crosstalk which causes physiopathology of bone with age. Myostatin, a muscular-secreted myokine, has been revealed to exert modulating effects on bone mass. Notably, it was found that downregulation of exosomal miR-218 derived from osteocytes could mediate inhibition of osteoblastic differentiation that is induced by myostatin through repression of sclerostin (SOST). ${ }^{401}$

Exosomal IncRNAs also exert important roles in osteoporosis. Teng et al. detected the levels of circulating exosomal IncRNAs in osteoporotic patients compared with normal subjects and identified 393 differentially expressed IncRNAs. ${ }^{402}$ Further bioinformatics analysis suggested that these IncRNAs may be associated with several osteoporosis pathways. ${ }^{402}$ Elsewhere, Cui et al. reported that IncRNA MALAT1 encapsulated by exosomes from endothelial progenitor cells (EPCs) could promote osteoclastogenesis of bone marrow-derived macrophages. ${ }^{403}$ This effect is achieved by MALAT1 serving as a miR-124 sponge to upregulate integrin subunit $\beta 1$ (ITGB1), which presents a pivotal role in osteoclastogenesis. ${ }^{403}$

Recently, exosomal circRNAs are recognized as a novel player in the pathophysiology of osteoporosis. CircRNAs in exosomes are differentially expressed in patients with osteoporosis. Among these circRNAs, exosomal hsa-circ-0006859 were upregulated and could differentiate individuals with osteopenia or osteoporosis from healthy individuals with high sensitivity and specificity. Mechanistically, hsa-circ-0006859 represses osteoblastic differentiation and induced adipogenic differentiation of human BMSCs through sponging miR-431-5p to promote ROCK1 expression. ${ }^{404}$ Exosomes containing a low concentration of circHmbox 1 derived from TNF-a-treated osteoclasts decrease osteoblasts differentiation mainly by targeting miR-1247-5p. Overexpression of circHmbox 1 remarkably mitigates the osteoporotic phenotypes in ovariectomized mice, which might function as a promising treatment strategy for postmenopausal osteoporosis. ${ }^{405}$

In a brief summary, exosomal miRNAs exert regulatory effects on osteogenesis, bone formation, and muscle-bone crosstalk. Exosomal IncRNAs are associated with a variety of osteoporosis pathways, such as MALAT1, which promotes osteoclastogenesis. Exosomal circRNAs are emerging as new regulators in the pathophysiology of osteoporosis. For instance, exosomal hsacirc-0006859 is a highly sensitive and specific marker for patients with osteoporosis, while exosomal circHmbox 1 exhibits a potential therapeutic effect for postmenopausal osteoporosis.

The roles of exosomal ncRNAs in cardiovascular diseases Despite the continuous progress in the treatment of cardiological ailments, cardiovascular diseases, such as hypertension, atherosclerosis (AS), acute myocardial infarction (AMI), heart failure (HF), and atrial fibrillation (AF), are still the leading cause of the morbidity and mortality worldwide. ${ }^{406,407}$ Recently, accumulating exosomal ncRNAs has been identified to be involved in the pathogenesis of cardiovascular diseases, which provides a new insight into the mechanisms and therapeutic targets for the diagnosis and treatment of these diseases. ${ }^{408-413}$ Moreover, exosomal ncRNAs serve as emerging regulators in dyslipidemia, thereby leading to an increase in risk of atherosclerotic cardiovascular diseases. ${ }^{414}$ For example, miR-26a is downregulated in circulating exosomes of overweight humans and obese mice, whereas, upregulation of miR-26a in mice reduces the abundance of plasma cholesterol, low-density lipoprotein (LDL) and HDL, hepatic triglyceride, as well as lipid droplets in adipose tissue. ${ }^{360}$ This study summarizes the current evidence about the roles of exosomal ncRNAs in cardiovascular diseases.

Exosomal ncRNAs in hypertension. Hypertension is an important risk factor for total mortality and cardiovascular disease, such as stroke, myocardial infarction, coronary heart disease, and HF. ${ }^{415-417}$ Emerging evidence indicates that diverse exosomal
ncRNAs were implicated in the development of hypertension through modulating multiple cellular and molecular events, including renin-angiotensin-aldosterone system (RAAS), endothelial dysfunction, angiogenesis, VSMCs proliferation, vascular remodeling, inflammation, and oxidative stress. ${ }^{418-420}$

Current evidence reveals that exosomal miRNAs from different sources can modulate the initiation and progression of hypertension. Next-generation sequencing was used to detect the exosomal miRNA expression profile in spontaneously hypertensive rats (SHRs) and normotensive Wistar-Kyoto rats (WKYs). Liu et al. found that 23 exosomal miRNAs were significantly upregulated and 4 exosomal miRNAs were downregulated in SHRs compared to WKYs. The levels of exosomal miR-17-5p and miR-425-5p were markedly elevated in SHRs plasma and tightly associated with inflammation and blood pressure homeostasis, respectively. ${ }^{421}$ Besides, exosomal miR-155-5p derived from aortic adventitial fibroblast was downregulated in SHRs compared to WKYs. Low expression of exosomal miR-155-5p enhanced the expression of vascular angiotensin-converting enzyme and angiotensin II (Ang II) and promoted VSMCs proliferation, vascular remodeling, and hypertension. ${ }^{422}$

Macrophages-derived exosomal miRNAs play crucial roles in hypertension. It has been reported that THP-1 cells-derived exosomal miR-27a impaired vasodilation and increased rat blood pressure through inhibiting the expression of Mas receptor in ECs and endothelial nitric oxide synthase (eNOS) phosphorylation in mesenteric arteries. ${ }^{423}$ Additionally, miR-17 was decreased in Ang II-treated THP-1-derived exosomes and promoted ECs inflammation through increasing the expression of intercellular adhesion molecule-1 (ICAM-1) and plasminogen activator inhibitor-1 (PAI-1). ${ }^{424}$ The transfer of exosomal miR-106b-5p from macrophages to renal juxtaglomerular cell stimulated inflammation-induced hypertension by inhibiting transcription factors E2f1 and Pde $3 b^{425}$

Exosomal ncRNAs in AS. AS is a chronic immune-inflammatory and age-related disorder which is characterized by lipid-rich plaques accumulated in the arterial wall. ${ }^{426}$ It is one of the main causes of cardiovascular diseases that leads to severe clinical outcomes like myocardial infarction and stroke. ${ }^{427-429}$ Accumulating evidence shows that various exosomal ncRNAs play an important regulatory role in the pathophysiological process of atherosclerosis. They were involved in the occurrence and development of atherosclerosis through regulating vascular inflammation, lipid metabolism, and cell survival. ${ }^{430-432}$

Several studies have demonstrated that exosomal miRNAs released by various types of cells are detected in circulation and are involved in the regulation of pathogenic AS. ${ }^{410,433}$ It has been reported that the levels of exosomal miR-223, miR-339 and miR-21 derived from thrombin-activated platelet were significantly upregulated. ${ }^{434,435}$ They could be transferred into VSMCs and inhibited the VSMCs proliferation stimulated by platelet derived growth factor. ${ }^{434}$ Besides, Li et al. also demonstrated that miR-223 inhibited TNF-a-stimulated endothelial cells (ECs) inflammation by decreasing the expression of ICAM- $1 .{ }^{435}$ These findings indicate that exosomal miR-223 may play a protective role in AS through inhibiting the vascular inflammatory response.

The communication between exosomal miRNAs and ECs plays a vital role in the pathogenesis of $\mathrm{AS}^{436}$ Exosomal miR-92a, upregulated by the combination of low shear stress and oxidized LDL in atherosclerotic mice model, promoted endothelial inflammation and atherosclerotic plaque formation. ${ }^{437}$ Besides, Xing et al. reported that exosomal miR-342-5p released by adiposederived MSCs exerted an anti-atherosclerotic effect by promoting $\mathrm{H}_{2} \mathrm{O}_{2}$-induced ECs apoptosis and protecting against ECs injury. ${ }^{438}$ On the contrary, miR-155 derived from VSMCs destroyed the tight junction and integrity of ECs, which led to the ECs injury and might promote AS progress. ${ }^{430}$

The dysfunction of VSMCs mediated by macrophages-derived exosomal miRNAs also plays an important role in AS. ${ }^{432,439}$ A study 
14

by Zhu et al. reported that the expression of exosomal miR-21-3p was increased in nicotine-stimulated macrophages and could be assimilated by neighboring VSMCs. ${ }^{432}$ This effect resulted in an increase in capacities of VSMCs proliferation and migration and thus accelerated the development of AS. Further, it was found that exosomal miR-106a-3p derived from oxidized LDL-incubated macrophages can promote VSMCs proliferation and inhibit their apoptosis, which may protect individuals against AS. ${ }^{439}$

Macrophages are the main types of cells responsible for the inflammation reaction in AS. The role of macrophages is regulated by exosomal miRNAs which has attracted a lot of attention. It has been found that miR-let7 family is highly enriched with exosomes released by MSCs and inhibited the macrophages infiltration via IGF2BP1 pathway. ${ }^{67}$ Besides, it also promotes the polarization of M2 macrophages via HMGA2/NF-KB pathway, which ameliorates the progression of $\mathrm{AS}^{67}$

In addition to miRNAs, it has been demonstrated that the expression of IncRNA GAS5 significantly increases in exosomes collected from patients or animals with $\mathrm{AS}^{440}$ Further in vitro study revealed that IncRNA GAS5 promoted the apoptosis of macrophages and ECs induced by oxidized LDL, which may lead to the aggravation of AS. ${ }^{440}$ Besides, the expression of MALAT1, a IncRNA involved in tumorigenesis, was downregulated in oxidized LDL-induced ECs. Overexpression of exosomal MALAT1 derived from ECs increases the expression of MALAT1 in dendritic cells and inhibits the production of ROS and maturation of dendritic cells through activating NRF2 signaling pathway. ${ }^{441}$ This indicates that downregulation of exosomal MALAT1 derived from oxidized LDLinduced ECs may accelerate the development of AS through inducing dendritic cells maturation. Intriguingly, another study found an increase in the expression of ECs-derived exosomal MALAT1, which promotes the M2 macrophage polarization and may protect individuals against AS. ${ }^{442}$ However, another study found that the increase in expression of exosomal MALAT1 derived from ECs promotes the formation of neutrophil extracellular traps and accelerates the progression of $\mathrm{AS}^{443}$

A few studies have found an association between exosomal circRNAs and the pathogenesis of AS. ${ }^{444,445}$ Wen et al. suggested that the expression of exosomal circRNA-0006896 in circulation is positively associated with the levels of TG, LDL, and C-reactive protein (CRP) in patients with unstable plaque atherosclerosis. ${ }^{445}$ The study also reported that the elevated circulating exosomal circRNA-0006896 leads to high expression of circRNA-0006896 in ECs, which results in an increases in capacities of proliferation and migration in ECs. This effect is accompanied with the upregulation of DNMT1 and phosphorylation of STAT3, which may provide protection against the vulnerable plaque formation. ${ }^{445}$

Exosomal ncRNAs in AMI. AMI is characterized by the loss of cardiomyocyte and the necrosis of myocardial. It results from acute obstruction of the coronary artery and can leads to severe clinical consequences. ${ }^{446}$ Recent studies have revealed that exosomal ncRNAs play a vital role in AMI and may become effective biomarkers for diagnosis and treatment of AMI. ${ }^{44-449}$

During the pathogenesis of $\mathrm{AMI}$, a wide range of exosomal miRNAs such as miR-125b, miR-499, miR-133, miR-22, miR-21, and miR-301 are found to be upregulated. ${ }^{450-454}$ Recent evidence has indicated that exosomal miRNAs are involved in the regulation of occurrence and development of AMI. This could be through regulation of pathophysiology pathways such as apoptosis, autophagy, inflammation, and angiogenesis. ${ }^{47,455-457}$

It has been demonstrated that miR-125b, miR-25-3p, miR-144, miR-126, and miR-146a encapsulated in exosomes from myocardial cells are enriched in expression and exert an antiatherosclerotic role partly by inhibiting myocardial apoptosis and facilitating ischemic cardiac repair. ${ }^{40,458-461}$ Besides, exosomal miR-25-3p and miR-146a derived from myocardial cells were also found to restrain the inflammation response, whereas exosomal miR-301 inhibits myocardial autophagy. ${ }^{454,458,461}$ On the contrary, it was reported that circulating levels of exosomal miR-499 and miR-133a derived from cardiomyocytes are elevated in patients with acute coronary syndromes than those with stable coronary artery disease or without coronary artery disease. ${ }^{451}$ This implies that exosomal miR-499 and miR-133a may promote the progression of AMI.

In addition, exosomal miRNAs released by other types of cells other than cardiomyocytes also plays an important role in the pathogenesis of AMI. According to Feng et al. the expression of miR-22 in exosomes from MSCs is upregulated under ischemic condition. ${ }^{452}$ It is also revealed that exosomal miR-22 could be internalized by cardiomyocytes and promote the protection of cardiomyocytes against apoptosis via targeting methyl-CpGbinding protein 2 in vitro. Further in vivo experiments shows that delivery of exosomal miR-22 remarkably reduces cardiac fibrosis thus indicating their significant benefit in treating myocardial fibrosis after AMI. ${ }^{452}$ Besides, Wang et al. reported that enhanced expression of exosomal miR-21 derived from MSCs have a cardiac protective role through inhibiting apoptosis and promoting angiogenesis of ECs. ${ }^{453}$

Emerging evidence demonstrates an involvement of exosomal IncRNAs in AMl. $^{448,449,462,463}$ According to Shyu et al. the expression of exosomal MALAT1 derived from hyperbaric oxygen-induced cardiomyocytes is significantly increased after AMI. ${ }^{448}$ The increase in MALAT1 then suppresses the expression of miR-92a and enhances the neovascularization. Huang et al. found an elevation in exosomal IncRNA H19 expression released by MSCs after treatment with atorvastatin. This led to an improvement of cardiac function recovery, a reduction of infarct size and cardiomyocyte apoptosis. ${ }^{449}$ Further, Wang et al. reported that the expression of exosomal IncRNA AK139128 from cardiomyocytes under hypoxic condition increases and this promotes the apoptosis but inhibits the proliferation, migration and invasion of cardiac fibroblasts. ${ }^{463}$

Exosomal ncRNAs in HF. HF results from dysfunction of myocardial systole or diastole. It is referred to as the decrease in cardiac blood output and the insufficient blood flow in the pulmonary or systemic circulation. ${ }^{464}$ The main mechanisms involved in HF include myocardial inflammation, autophagy, apoptosis, and remodeling. Recent studies have revealed that exosomal ncRNAs are involved in the regulation of pathogenesis of HF. ${ }^{462,463,465}$

During the pathogenesis of HF, a number of exosomal miRNAs are reported to be altered in the serum levels. It has been reported that exosomal miR-425 and miR-744 are lowered in the circulation of patients with HF. ${ }^{466}$ The downregulation of exosomal miR-425 and miR-744 are also reported in angiotensin II-treated cardiac fibroblasts and is positively related to the expression of collagen 1 and a-SMA, which leads to cardiac fibroblast fibrosis. Further in vitro experiments have revealed that exosomal miR-425 and miR-744 protect against myocardial remodeling by inhibiting angiotensin-II-induced collagen formation and fibrogenesis by targeting TGF $\beta 1 .{ }^{466}$

Exosomal ncRNAs in AF. AF is a type of cardiac arrhythmia and is correlated with the structural and electrical remodeling. ${ }^{467,468}$ Recently, some studies have revealed that ncRNAs, especially miRNAs play an important role in pathological process of AF. ${ }^{469-471}$ According to Liu et al. there is low expression of miR$320 \mathrm{~d}$ in AF cardiomyocytes, accompanied with an increase in apoptosis and impaired cell viability in cardiomyocytes. ${ }^{469}$ However, transfecting with exosomal miR-320d derived from adipose tissue-derived mesenchymal stem cells significantly reverses the effects of AF on cardiomyocytes, which is dependent on inhibition of STAT3. Besides, Wang et al. reported that the expression of exosomal miR-107 derived from patients with AF was significantly higher than the expression from patients without 
AF. ${ }^{470}$ Incubation of ECs with AF-derived exosomes or miR-107 mimics significantly inhibits cell viability and migration, while enhancing cell apoptosis by regulating miR-107/USP14 pathway. Furthermore, $\mathrm{Li}$ et al. demonstrated that miR-21-3p loaded in exosomes derived from myofibroblasts contributes to an increase in vulnerability of AF through upregulation of the expression of L-type calcium channel Cav1.2. ${ }^{471}$

In summary, exosomal ncRNAs are identified as key regulators in diverse cardiovascular diseases such as hypertension, AS, AMI, $\mathrm{HF}$, and AF. Exosomal miRNAs are relatively well studied in cardiovascular diseases, which exert functions by inhibiting vascular inflammatory response, modulating cellular pathophysiology pathways, and so on. Exosomal IncRNAs (GAS5, MALAT1, H19, AK139128) are associated with AS and AMI. However, only a small number of exosomal circRNAs have been determined to be involved in the pathogenesis of AS.

The roles of exosomal ncRNA in neurodegenerative diseases The incidence of neurodegenerative diseases, including Alzheimer's disease (AD) and Parkinson's disease (PD) increases with age. Deviation of ncRNA levels in neurodegenerative diseases has been widely reported. ${ }^{472-478}$ Exosomes are demonstrated to carry ncRNAs and establish cell-to-cell communication in neurons. They are involved in the pathogenesis and progression of neurodegenerative diseases as mediators.

Exosomal ncRNA in $A D$. $A D$ is the most common cause of dementia in elderly people. The pathological features of $A D$ are extracellular $A \beta$ deposition, intracellular neurofibrillary tangles, and neuronal loss. ${ }^{479}$ Exosomal ncRNAs have been shown to regulate the expression and function of amyloid precursor proteins (APP) and tau proteins. ${ }^{480}$

Growing evidence shows that exosomal miRNAs play an important role in $A D .^{481,482}$ MiRNA expression profile alters in the brains of patients with $A D .^{483} A P P, \beta$-site amyloid precursor protein-cleaving enzyme 1 (BACE1) and microtubule-associated protein tau (MAPT) are pathologically related proteins of AD. ${ }^{484,485}$ Neuropathological changes in $A D$ are the consequence of diverse cellular processes, such as alterations of $A D$ relevant proteins and oxidative stress.

The interaction between exosomal miRNAs and APP plays an important role in the pathogenesis of AD. Mir-34a, a miRNA strongly related to cognitive dysfunction, is highly expressed in postmortem brain tissue of $A D$ patients. It has been found that miR-34a promotes amyloid processing of APP, whereas knockdown of miR-34a reduces APP accumulation in brain tissues. ${ }^{486,487}$ Exosomes secreted by miR-34a-overexpressing neurons can be absorbed by adjacent neurons, resulting in the inhibition of target genes in neural network. ${ }^{488}$ Overexpression of miR-34a in AD tissues is correlated with simultaneous inhibition of target genes of synaptic plasticity, oxidative phosphorylation, and glycolysis. Moreover, miR-193b can also target the $3^{\prime}$ untranslated regions of mRNA (UTR) of APP to exert a regulatory role and further influence the progression of $A D$. The peripheral blood-derived exosomal miR-193b was measured in the normal population, patients with mild cognitive impairment $(\mathrm{MCl})$ and patients with $A D$, and it was found that miR-193b is significantly lower in patients with $\mathrm{MCl}$ and $A D$ than in the control group and its expression level was negatively correlated with $A \beta 42 .{ }^{489}$

Several research reports have clarified the roles of oxidative stress in the pathogenesis of neurodegenerative diseases, ${ }^{490-492}$ because oxidative stress has been shown to play an important role in enhancing beta-amyloid and tau hyperphosphorylation. ${ }^{493}$ Low concentrations of miR-141-3p have been observed in plasma exosomes from patients with AD. ${ }^{494}$ A large amount of miR-141-3p was also found in the exosomes of astrocytes stimulated by inflammation. ${ }^{495}$ Further studies have confirmed that miR-141-3p can damage the antioxidant defense system and upregulate oxidative stress by inhibiting PTEN. ${ }^{496}$ In addition, exosomal miR-125b-5p is also involved in the process of $A D .^{497}$ Transfection of miR-125b significantly promoted neuronal apoptosis and Tau phosphorylation by activating cyclin-dependent kinase 5 (CDK5) and p35/25. ${ }^{498}$ Besides, inhibition of miR-125b-5p reduces ROS levels, showing a neuroprotective effect against oxidative stress. ${ }^{499}$

Exosomal IncRNAs also take part in the pathogenesis of $A D$. LnCRNA BACE1-AS is upregulated in the brain of patients with AD. The levels of IncRNA BACE1-AS in plasma derived exosomes of $A D$ and healthy individuals have been determined. No significant difference was observed between the two groups. However, in the whole plasma sample, there was significant difference between $A D$ and the control groups. ${ }^{500}$

Exosomal ncRNA in PD. PD is characterized by bradykinesia, resting tremor and postural and gait disorders. Typical pathological features of PD are loss of dopaminergic (DAergic) neurons in the dense part of substantia nigra and aggregation of a-SYN protein in Lewy bodies and neurites. ${ }^{501,502}$ Exosomes-derived ncRNAs are involved in the pathophysiology of PD. ${ }^{503-506}$

Multiple miRNAs are reported to be upregulated in exosomes of PD cell model, including miR-210-5p, miR-128-1-5p, miR-505-5p, miR-325-5p, miR-16-5p, miR-1306-5p, miR-669b-5p, miR-125b5p, miR-450b-3p, miR-24-2-5p, and miR-2 -6516-3p and miR-1291. These exosomal miRNAs regulate important pathways in the pathogenesis of PD, such as autophagy, inflammation, and protein aggregation. ${ }^{507}$

Similarly, changes in exosomal miRNAs are found in the cerebrospinal fluid (CSF) of PD patients. Among them, exosomal miR-153, miR-409-3p, miR-10a-5p, and let-7g-3p were significantly increased in CSF of PD patients, while miR-1 and mir19b-3p were significantly decreased. ${ }^{508}$ Let-7 miRNA family is highly conserved in animal species. It has been reported that let-7 was highly expressed in the PD model. ${ }^{509}$ The expression of exosomal let-7 in CSF of PD patients was upregulated. ${ }^{510}$ When exosomal let-7 is absorbed by neurons, it leads to neurodegenerative changes by activating TLR7. ${ }^{511}$ In addition, in C. elegans PD model, silence of let-7 decreases the accumulation of a-SYN protein, thereby alleviating the progression of PD..$^{512}$

In addition, the levels of exosomal miRNAs altered in the circulating plasma of PD patients. MiR-195, miR-24, and miR-331$5 p$ were upregulated whereas miR-19b and miR-505 were downregulated in circulating exosomes of patients with PD. ${ }^{513,514}$ Moreover, the increase of miR-137 was observed in the plasma of PD patients. ${ }^{515,516}$ Downregulation of exosomal miR-137 can upregulate oxidative resistance 1 (OXR1) in PD mice model, thereby generating a neuroprotective effect. ${ }^{517}$

When the level of IncRNA extracted from exosomes in plasma samples from PD patients and control group was measured, it was found that 15 and 24 exosomal IncRNAs were upregulated and downregulated, respectively. Among them, Inc-MKRN2-42:1 was positively correlated with MDS-UPDRS III score, which is used to evaluate the severity of dyskinesia in PD patients. ${ }^{518}$ Compared with the control group, the concentrations of Inc-POU3F3 and aSYN increased in neuro-derived L1CAM exosomes in PD patients. There was a significant correlation between L1CAM exosomal IncPOU3F3 levels and PD severity, including motor/cognitive impairment. $^{519}$ Four IncRNAs (SNCA-AS1, MAPT AS1, AK127687, and AX747125) were detected in exosomes from human cerebrospinal fluid, providing preliminary evidence that these IncRNAs may be of potential use as a diagnostic tool for PD. ${ }^{520}$ However, further research is needed to elucidate their possible role in PD.

In this chapter, we review recent research on the roles of ncRNAs, especially miRNAs and IncRNAs, in neurodegenerative diseases with an emphasis on $A D$ and PD, whereas exosomal circRNAs have been poorly studied. This fascinating area needs to be explored further. 
16

The roles of exosomal ncRNA in autoimmune diseases

Autoimmune diseases refer to the diseases caused by the immune response of human body to its own antigens hence causing damage to its own tissues. Rheumatoid arthritis (RA) and systemic lupus erythematosus (SLE) are the most common systemic autoimmune diseases. The pathogenesis of autoimmune diseases is complex and a lot about it is still unknown. The current research studies shows that ncRNAs encapsulated in exosomes play a critical role in autoimmune diseases.

Exosomal ncRNA in RA. RA is a chronic autoimmune disease characterized by infiltration of leukocyte into joints, causing production of inflammatory mediators and destruction of bone and cartilage tissue. ${ }^{521}$ Synovitis mediated articular cartilage destruction is associated with upregulation of matrix metalloproteinases (MMPs). This cartilage injury is irreversible and forms a key step in RA joint injury. ${ }^{522}$ Angiogenesis mediates the delivery of nutrients and inflammatory factors. Continuous angiogenesis leads to chronic changes of synovium in RA. ${ }^{523}$ Part of the role of MMPs in regulating vascular remodeling is that they activate the secretion of VGF- $\beta$ in the stromal matrix by activating VEGF. ${ }^{524}$

MiR-150-5p is associated with T cell maturation and is therefore involved in autoimmune diseases. ${ }^{525}$ It was also confirmed that miR-150-5p could regulate angiogenesis. ${ }^{526}$ Compared with patients with osteoarthritis, there is a decrease in expression of miR-150-5p and an increase in expression of VEGF and MMP-14 as well as angiogenesis in RA patients. ${ }^{527}$ The previous study showed that exosomal micRNA-150 alleviates RA symptoms by downregulating MMP14 and VEGF as well as inhibiting angiogenesis. ${ }^{527}$ In addition, the miRNA microarray analysis showed that miR-548a$3 p$ was significantly reduced in serum exosomes of RA patients. Serum exosomal miR-548a-3p was negatively correlated with serum levels of CRP, rheumatoid factor (RF), and erythrocyte sedimentation rate (ESR) in RA patients. Further research has proven that exosomal miR-548a-3p is involved in the regulation of macrophage mediated inflammation through the TLR4/NF-KB signaling pathway in RA. Therefore, exosomes may be an important factor in predicting RA disease activity. ${ }^{528}$

LncRNAs profiles in RA serum exosomes were analyzed, and it was found that Hotair, LUST, anti-NOS2a, MEG9, SNHG4, TUG1, and NET1 were upregulated in RA serum exosomes and Hotair expression level was increased by an average of about 4 times in RA exosomes. Further studies confirmed that the overexpression of Hotair in RA exosomes may be involved in the pathogenesis of RA in two ways. First, it attracts activated macrophages and induces an immune response. Secondly, Hotair may be involved in the production of MMP in osteoclasts and RA synovial cells. ${ }^{529}$

Exosomal nCRNA in SLE. SLE is an autoimmune disease involving multiple systems and organs, which is characterized by persistent inflammation and autoantibody production. MiRNAs carried by exosomes are involved in the pathogenesis of SLE, especially in regulating inflammation and immune imbalance.

Exosomal miRNAs play an important role in the immunepathophysiology of SLE. ${ }^{50,531}$ Dysregulation of miRNA has been found in SLE, and exosomal miRNA from patients with SLE can regulate inflammation and adaptive immune response. ${ }^{532}$ Previous studies have found that miR-155 promotes autoimmune process by inhibiting suppressor of cytokine signaling-1 (SOCS$1)^{533}$ or by repressing transcription of PU.1 and TNF-a. ${ }^{534}$ Exosomal miR-155 expression was significantly upregulated in patients with SLE, which was higher in patients with lupus nephritis (LN). ${ }^{535}$ Defects in miR-155 ameliorate autoimmune inflammation in SLE. ${ }^{536}$ Moreover, plasma miR-21 levels in patients with SLE were higher than with healthy controls. ${ }^{537}$ Further studies have confirmed that miR-21 modulates abnormal T-cell responses in SLE patients. ${ }^{538}$ MiR-21 was significantly upregulated in exosomes of SLE patients. ${ }^{535}$ Moreover, exosomal miR-21 from
SLE patients can induce the production of type I interferon by dendritic cells. $^{532}$

The expression of serum exosomal miR-451a is correlated with SLE disease activity and renal injury. The level of serum exosome miR-451a in patients with active SLE was significantly lower than that in patients with inactive SLE and control group, especially in those with renal damage. Glucocorticoid or hydroxychloroquine increases the expression of exosomal miR-451a in CD4 ${ }^{+}$T cells. $^{539}$ The aging of MSCs plays an important role in the occurrence and progression of SLE. The expression of miR-146a in serum exosomes of patients with SLE decreases significantly compared with healthy controls. MiR-146a is internalized into MSCs through exosomes and is involved in MSCs senescence by targeting TRAF6/NF-KB signaling pathway. ${ }^{540}$ In addition, it also found that miR-146a was associated with SLE activity and proteinuria. ${ }^{541}$

LN occurs in 40 to $75 \%$ of patients with SLE, which is one of the leading causes of death in these patients. ${ }^{542}$ Compared with other biological samples, urine samples are easy to obtain hence less costly. Urinary exosomal miRNAs can accurately reflect renal dysfunction and structural damage. ${ }^{543,544}$ Multiple urinary exosomal miRNAs have been found to be associated with renal fibrosis, ${ }^{545,546}$ and three of these were associated with renal fibrosis in LN, including miR-410, miR-29c, and miR-150. ${ }^{547-549}$ Among them, miR-410 directly interacts with IL-6 3'- UTR to reduce its expression and inhibits renal fibrosis ${ }^{548}$ whereas miR-150 promotes renal fibrosis by downregulating SOCS1. ${ }^{547}$ MiR-29C levels were inversely correlated with histological chronicity index and glomerulosclerosis, but not with renal function. ${ }^{549}$ In addition, let-7 and miR-21 were downregulated in patients with active LN compared with patients with inactive LN. It was reported that let-7 and miR-21 are downregulated during disease flare and upregulated after treatment. It is suggested that urinary exosomal miRNAs (let-7a and miR-21) can be used to guide the clinical staging of patients with LN. ${ }^{550}$

In this section, we discuss the roles of exosomal ncRNAs in autoimmune diseases including RA and SLE. Exosomal miR-150-5p alleviates RA symptoms by regulating VEGF, MMP-14, and angiogenesis, while exosomal miR-548a-3p regulates macrophage-mediated inflammation in RA. Exosomal IncRNAs Hotair, LUST, anti-NOS2a, MEG9, SNHG4, TUG1, and NET1 may be involved in the pathogenesis of RA. In addition, exosomal miRNAs have been implicated in the pathogenesis of SLE, especially in the regulation of inflammation and immune imbalance, activity, and complication, mainly associated with LN.

The roles of exosomal ncRNAs in infectious diseases Infectious diseases refer to a group of diseases that are caused by viruses, bacteria, fungi, or other pathogens. Among many infectious diseases, pneumonia and viral hepatitis are very common in the human population. Recently, accumulating evidence revealed that exosomal ncRNAs participated in the pathogenesis of infectious diseases. ${ }^{551-553}$ In this study, we summarize the current evidence about the roles of exosomal ncRNAs in pneumonia and viral hepatitis.

Exosomal ncRNAs in pneumonia. Pneumonia is a very common disease worldwide. Severe community-acquired pneumonia remains a life-threatening disease especially in children and elderly. ${ }^{554}$ It has been demonstrated that exosomal ncRNAs levels are markedly different in patients with pneumonia. ${ }^{553,555}$

Exosomal miRNAs are the most studied ncRNAs involved in the development of pneumonia. Recently, it was reported that abundant exosomes selectively loaded with miR-155 are presented in circulation from sepsis-related acute lung injury (ALI) mice. ${ }^{555}$ Injection of exosomes harvested from ALI mice significantly increased the number of M1 macrophages in the lung and led to the inflammation in healthy mice through activating the NF$\mathrm{KB}$ signaling pathway and the downstream upregulation of TNF-a 


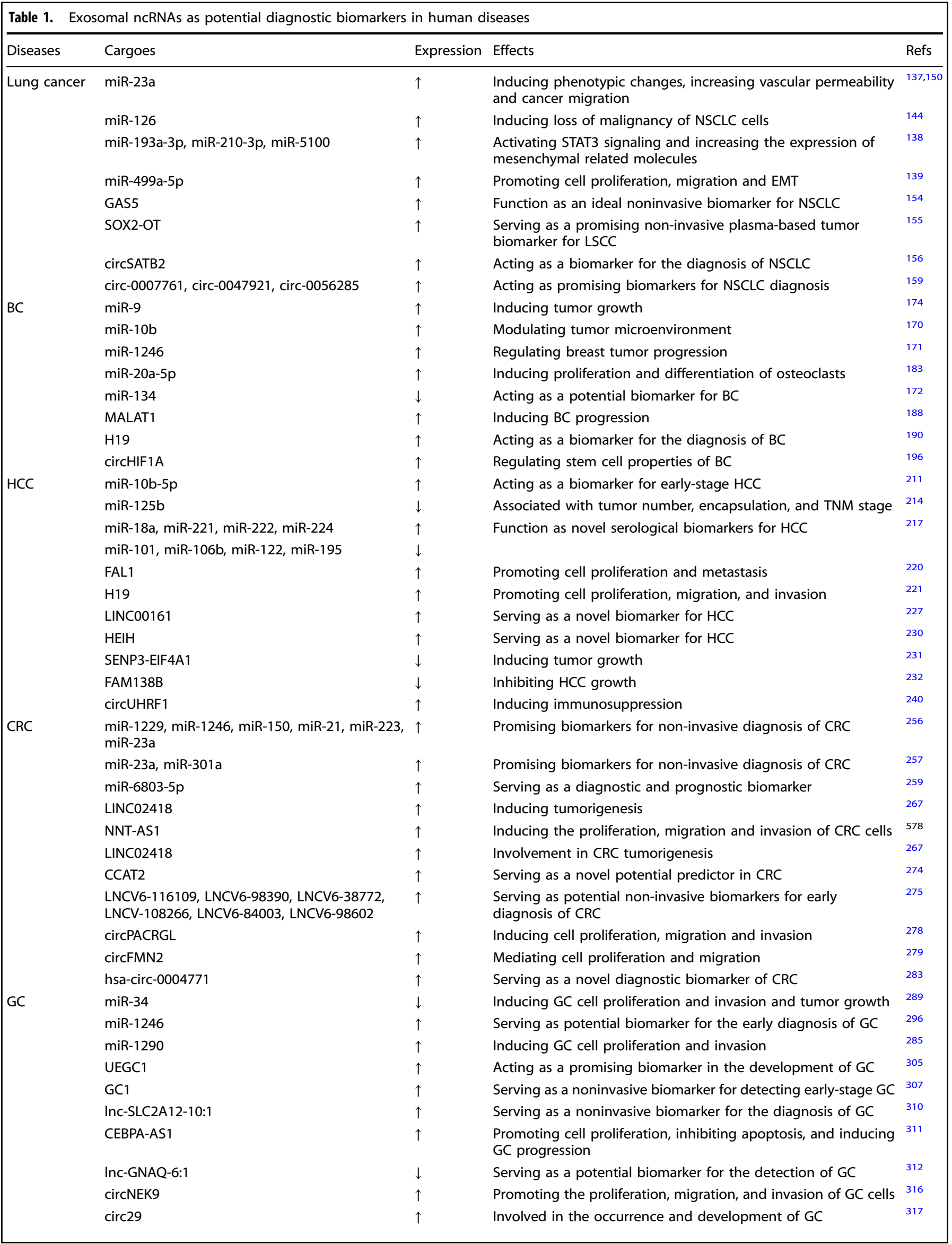




\begin{tabular}{|c|c|c|c|c|}
\hline \multirow{2}{*}{ PCa } & has-circ-0065149 & $\downarrow$ & Acting as a novel biomarker for diagnosis & 320 \\
\hline & circ-0044516 & $\uparrow$ & Inducing tumor cell metastasis & 337 \\
\hline T2DM & miR-20b-5p & $\uparrow$ & Attenuating insulin-stimulated glycogen accumulation & 363 \\
\hline MetS & MALAT1 & $\downarrow$ & $\begin{array}{l}\text { Serving as a potential epigenetic biomarker of diabetes risk } \\
\text { or MetS }\end{array}$ & 366 \\
\hline \multirow[t]{2}{*}{ OP } & miR-214 & $\uparrow$ & Repressing osteoblasts activity & 395 \\
\hline & hsa-circ-0006859 & $\uparrow$ & Serving as a high sensitivity and specificity biomarker & 404 \\
\hline \multirow[t]{2}{*}{ Hypertension } & miR-155-5p & $\downarrow$ & Promoting VSMCs proliferation and vascular remodeling & 422 \\
\hline & miR-27a & $\uparrow$ & Promoting vasodilation and causing hypertension & 423 \\
\hline \multirow{8}{*}{ AS } & miR-92a & $\uparrow$ & $\begin{array}{l}\text { Promoting ECs inflammation and the formation of } \\
\text { atherosclerotic plaque }\end{array}$ & 437 \\
\hline & $\operatorname{miR}-342-5 p$ & $\uparrow$ & Promoting $\mathrm{H}_{2} \mathrm{O}_{2}$-induced ECs apoptosis & 438 \\
\hline & miR-155 & $\uparrow$ & Inhibiting ECs proliferation and migration & 430 \\
\hline & $\operatorname{miR}-21-3 p$ & $\uparrow$ & Promoting VSMCs proliferation and migration & 432 \\
\hline & $\operatorname{miR}-106 a-3 p$ & $\uparrow$ & Promoting proliferation and inhibiting apoptosis in VSMCs & 439 \\
\hline & GAS5 & $\uparrow$ & Promoting the apoptosis of macrophages and ECs & 440 \\
\hline & MALAT1 & $\uparrow$ & $\begin{array}{l}\text { Promoting the } \mathrm{M} 2 \text { macrophage polarization, the formation of } \\
\text { neutrophil extracellular traps in neutrophils }\end{array}$ & 442,443 \\
\hline & circRNA-0006896 & $\uparrow$ & Promoting proliferation and migration of ECs & 445 \\
\hline \multirow[t]{4}{*}{ AMI } & $\operatorname{miR}-125 b$ & $\uparrow$ & $\begin{array}{l}\text { Ameliorating cardiomyocytes apoptosis and facilitating } \\
\text { ischemic cardiac repair }\end{array}$ & 450 \\
\hline & MALAT1 & $\uparrow$ & Enhancing neovascularization & 448 \\
\hline & $\mathrm{H} 19$ & $\downarrow$ & Reducing infarct size and cardiomyocyte apoptosis & 449 \\
\hline & IncAK139128 & $\downarrow$ & $\begin{array}{l}\text { Promoting the apoptosis and inhibiting the proliferation, } \\
\text { migration, and invasion of cardiac fibroblasts }\end{array}$ & 463 \\
\hline $\mathrm{HF}$ & miR-425, miR-744 & $\downarrow$ & Protecting against myocardial remodeling & 466 \\
\hline \multirow[t]{2}{*}{$\mathrm{AF}$} & miR-320d & $\downarrow$ & Promoting viability and inhibiting apoptosis of cardiomyocytes & 469 \\
\hline & miR-107 & $\uparrow$ & $\begin{array}{l}\text { Suppressing viability and migration of ECs, enhancing cell } \\
\text { apoptosis }\end{array}$ & 470 \\
\hline \multirow[t]{3}{*}{$A D$} & $\operatorname{miR}-34 a$ & $\uparrow$ & Promoting amyloid processing of APP & 486,487 \\
\hline & miR-141-3p & $\uparrow$ & $\begin{array}{l}\text { Damaging the antioxidant defense system and up-regulating } \\
\text { oxidative stress }\end{array}$ & 496 \\
\hline & miR125b-5p & $\uparrow$ & Promoting neuronal apoptosis and Tau phosphorylation & 498 \\
\hline PD & let-7 & $\uparrow$ & Leading to neurodegenerative changes & 511 \\
\hline \multirow[t]{2}{*}{ RA } & $\operatorname{miR}-548 a-3 p$ & $\downarrow$ & Regulating macrophage mediated inflammation & 528 \\
\hline & Hotair & $\uparrow$ & Inducing an immune response & 529 \\
\hline \multirow[t]{2}{*}{ SLE } & miR-155 & $\uparrow$ & Regulating autoimmune inflammation in SLE & 536 \\
\hline & $\operatorname{miR}-21$ & $\uparrow$ & Inducing the production of type I interferon & 532 \\
\hline \multirow[t]{4}{*}{ Pneumonia } & $\operatorname{miR}-155$ & $\uparrow$ & Promoting inflammation and proliferation & 555 \\
\hline & $\operatorname{miR}-371 b-5 p$ & $\uparrow$ & $\begin{array}{l}\text { Promoting proliferation and re-epithelialization of injured } \\
\text { alveoli }\end{array}$ & 556 \\
\hline & miR-221/222 & $\uparrow$ & Promoting epithelial cell proliferation & 557 \\
\hline & $\operatorname{miR}-223 / 142$ & $\uparrow$ & Inhibiting LPS-induced lung inflammation & 558 \\
\hline
\end{tabular}


Roles and mechanisms of exosomal non-coding RNAs in human health and... Li et al.

Table 1. continued

\begin{tabular}{lllll}
\hline Diseases & Cargoes & Expression & Effects & \\
\hline Viral hepatitis & miR-221-3p, miR-25-3p & $\uparrow$ & Associated with liver fibrosis and inflammation \\
& miR-372-3, miR-10a-5p & $\downarrow$ & Associated with liver fibrosis and inflammation \\
& miR-19a & $\uparrow$ & Promoting liver fibrosis & 563 \\
\end{tabular}

NSCLC non-small cell lung cancer, STAT3 signal transducer and activator of transcription 3, EMT epithelial-mesenchymal transition, $L S C C$ lung squamous cell carcinoma, $B C$ breast cancer, $H C C$ hepatocellular carcinoma, $C R C$ colorectal cancer, GC gastric cancer, $P C a$ prostate cancer, $T 2 D M$ type 2 diabetes mellitus, $B M I$ body mass index, MetS metabolic syndrome, OP osteoporosis, AS atherosclerosis, VSMCs vascular smooth muscle cells, ECs endothelial cells, AMI acute myocardial infarction, $H F$ heart failure, $A F$ atrial fibrillation, $A D$ Alzheimer's disease, $A P P$ amyloid precursor proteins, $P D$ Parkinson's disease, RA rheumatoid arthritis, SLE systemic lupus erythematosus, LPS lipopolysaccharide

and IL-6. Besides, circulating exosomal miR-155 was also found to promote macrophage proliferation and inflammation by regulating SHIP1 and SOCS1. ${ }^{555}$ According to Quan et al. the expression of exosomal miR-371b-5p derived from injured alveolar progenitor type II cells is upregulated and decreases the expression of PTEN. ${ }^{556}$ This promotes cell proliferation and re-epithelialization of injured alveoli through activating phosphorylation of Akt and its substrates GSK3 $\beta$ and FOXOs. Importantly, the elevated levels of exosomal miR-371b-5p were also observed in lavage samples from patients with acute pneumonia, which may provide a potential therapeutic target for acute pneumonia.

Dysregulated immune reaction is a major contributing factor to the pathophysiology of pneumonia and is associated with the high incidence ratio of pneumonia. It was found that exosomal miR-221 and miR-222 levels derived from lipopolysaccharide (LPS)-induced macrophage increases and promotes the proliferation of epithelial cells. ${ }^{557}$ Besides, macrophages induced by LPS also releases high number of exosomes that deliver miR-223 and miR-142 hence inhibiting the LPS-triggered inflammatory responses in the lung. ${ }^{558}$ However, the expression of exosomal miR-103a-3p from LPS-induced lung cells and from circulation of pneumonia patients were decreased. Overexpression of exosomal miR-103a-3p attenuates inflammation by regulating immune response via transducing $\beta$-like $1 \mathrm{X}$ related protein $1 / \mathrm{NF}-\mathrm{kB}$ signaling pathway. ${ }^{559}$

Exosomal ncRNAs in viral hepatitis. Viral hepatitis is an infectious disease caused by a variety of hepatitis viruses. If not treated, it can develop into liver cirrhosis or even liver cancer which is very dangerous for human health. Therefore, exploration of the underlying mechanisms of viral hepatitis is of great significance. Accumulating evidence demonstrates that exosomal ncRNAs are involved in the pathogenesis of viral hepatitis. ${ }^{560}$

Exosomal miRNAs has been demonstrated to be involved in the regulation of multiple signaling pathways during the development of viral hepatitis. ${ }^{561,562}$ Globally, hepatitis B virus (HBV) is the most common hepatitis and causes very heavy economic and heathy burden. According to $\mathrm{Li}$ et al. some exosomal miRNAs, such as miR-221-3p and miR-25-3p are upregulated in patients with chronic HBV infection, whereas other such as miR-372-3 and miR$10 a-5 p$ are downregulated. ${ }^{563}$ Besides, miR-122, miR-204, miRlet7c, miR-23b, and miR150 are also altered and are correlated with HBV-related liver diseases. ${ }^{562}$

Hepatitis $C$ is also common in the human population. The fibrosis of the liver is correlated with the chronic infection of hepatitis $\mathrm{C}$ virus (HCV). However, the specific mechanisms remain poorly understood. Recently, Devhare et al. reported that the exosomal miR-19a released by HCV-infected hepatocytes is upregulated and increases the expression of fibrosis marker genes in hepatic stellate cells (HSC). ${ }^{564}$ Further analysis revealed that the exosomal miR-19a aggravates the liver fibrosis by activating the SOC3/STAT3/TGF- $\beta$ signaling pathway.

Relatively, there are few studies that evaluated exosomal IncRNAs in the development of viral hepatitis. ${ }^{565}$ It was found that the expression of runt-related transcription factor 1 (RUNX1) and RUNX1 overlapping RNA (RUNXOR) are significantly upregulated in myeloid-derived suppressor cells (MDSCs) during chronic HCV infection. These were positively correlated with the immunosuppressive molecule levels. ${ }^{565}$ Mechanistically, it has been demonstrated that HCV-associated exosomes deliver RUNXOR and RUNX1 to MDSCs and promote the function of differentiation and suppressive functions of MDSCs through regulation of STAT3 signaling pathway. These results indicate that RUNXOR and RUNX1 may become a promising target for immunomodulation with antiviral treatment during HCV infection.

In summary, the roles of exosomal miRNAs in pneumonia has been studied most intensively, among which miR-155 and miR$371 \mathrm{~B}-5 \mathrm{p}$ are related to the occurrence and development of pneumonia, while miR-221, miR-222, miR-223, miR-142, and miR-103a-3p are associated with the immune response of pneumonia. As for viral hepatitis, exosomal miRNAs have been shown to regulate multiple signaling pathways, whereas few exosomal IncRNAs (RUNXOR and RUNX1) have been demonstrated to be involved in the development of viral hepatitis.

\section{CLINICAL APPLICATIONS OF EXOSOMAL NCRNAS IN HUMAN DISEASES}

Recently, exosomal ncRNAs have emerged as novel players in the occurrence and development of various human diseases. Circulating ncRNA profiles in exosomes are altered for a wide range of diseases, suggesting that exosomal ncRNAs may serve as cycling indicators of an individual's physiological status and targeted therapies tools in precision medicine. Therefore, exosomal ncRNAs exert potential to be candidate biomarkers and therapeutic targets for the diseases. This section summarizes the present knowledge on the potential clinical implication of exosomal ncRNAs in biomarker identification and therapy exploration in diseases.

Exosomal ncRNAs as diagnostic biomarkers in human diseases Biomarker are a series of molecules that can be utilized for disease detection and/or prognosis prediction. Sensitivity, specificity, stability, and relatively non-invasive are most important and necessary characteristics of a good biomarker. Notably, emerging research is targeting exosomes as potential biomarkers because they can be detected using simple and inexpensive methods in many body fluids and are stable against enzyme degradation by providing a protective transportation system. It is worth noting that exosomal ncRNAs are differentially expressed in human lifestyle activities and various pathological diseases, including cardiovascular, metabolic, neurodegenerative, autoimmune, infectious, and cancerous disorders, which underlies the potential of ncRNAs as promising biomarkers for early detection. ${ }^{360,364,566-569}$ This study compiles the circulating exosomal ncRNAs that have been reported to be altered during the course of human diseases, as detailed in Table 1. 

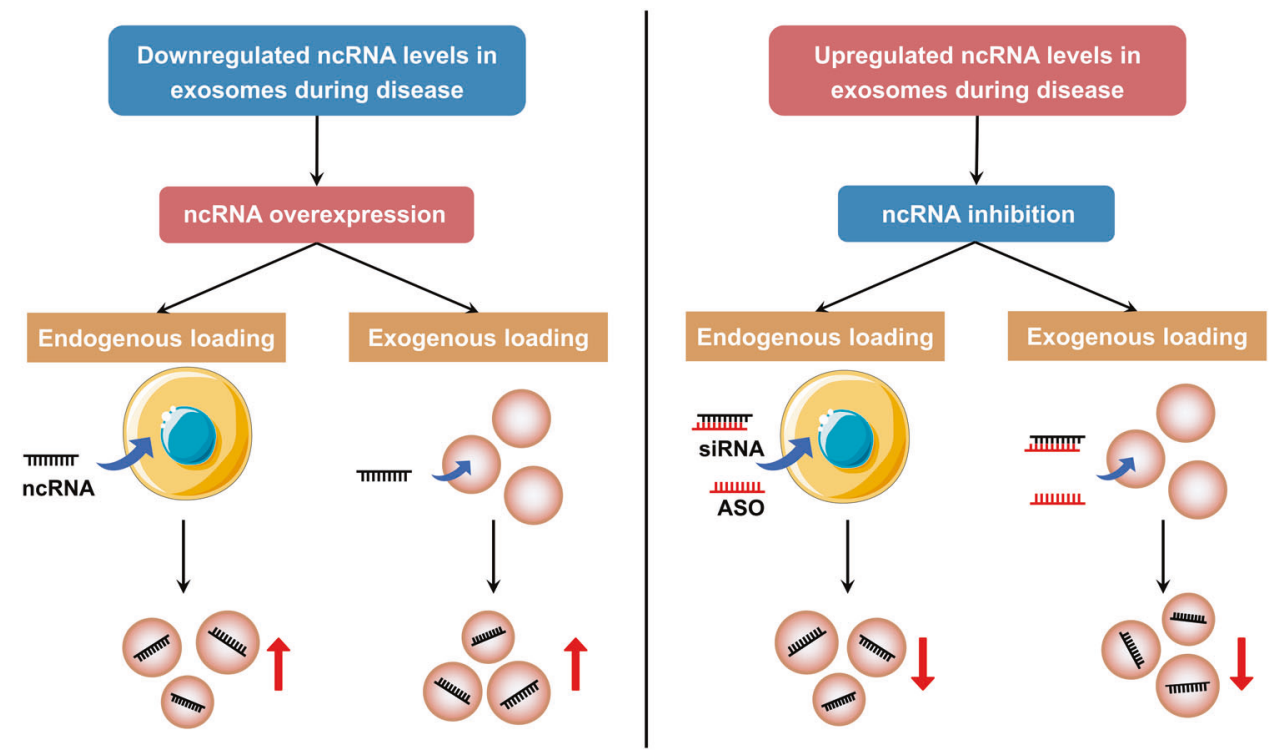

Fig. 7 Exosomal ncRNA-based therapeutics in human diseases. Endogenous (pre-loading cargoes into donor cells followed by exosomal cargo release) or exogenous (directly loading cargoes into exosomes after their production or isolation) loading of ncRNAs or their inhibitors into exosomes exhibits significant therapeutic potential. ASO antisense oligonucleotide. This figure was created with the aid of Servier Medical Art (https://smart.servier.com/)

Exosomal ncRNAs-based therapeutics in human diseases Considering the various advantages of exosomes, such as natural availability, considerate biocompatibility, biological barrier permeability, low immunogenicity, and toxicity, exosomes are poised to become a promising tool for therapeutic vehicles. ${ }^{7,570,571}$ Genetic engineering has been explored to modify exosomes for therapeutic utilization. The ability to modify isolated and purificatory exosomes loaded with mimics or inhibitors of ncRNAs and target molecules on their surface, offers the possibility of using exosomes as vectors to transport specific ncRNAs to specific tissues or organs (Fig. 7). This gene regulation proposes exosomal ncRNAs as promising approach for the treatment of various human diseases.

Therapies that upregulate exosomal ncRNAs. Exosomes can load ncRNAs release of which may present promising therapeutic effects. Several studies have explored important therapeutic interventions of exosome-based ncRNAs. By modifying exosomes with ncRNA mimics or inhibitors, exosomes can serve as delivery system of specific ncRNA sequences.

Elevated expression levels of beneficial ncRNAs carried by exosomes have been described to have various therapeutic implications. The levels of ncRNAs in exosomes will enhance by loading ncRNAs into exosomes endogenously or exogenously. Exogenous loading is performed after exosome production or isolation with cargo of interest encapsulated to exosomes by coincubation, chemical transfection, or physical approaches such as electroporation and dialysis. ${ }^{572}$ For example, Lv et al. used electroporation to package miR-21-5p mimics into ADSC-derived exosomes. The results of their study suggested that the miR-21-5p mimics-enriched exosomes accelerate diabetic wound healing in vivo through promoting re-epithelialization, collagen remodeling, angiogenesis, and vessel maturation. ${ }^{573}$

In addition, cargoes can be loaded in an endogenous way by pre-loading donor cells with therapeutic ncRNA, which will then be encapsulated in exosome. For instance, Zhuo et al. transfected IncRNA FAM138 into HCC cells and then created exosomes enriched in FAM138, which alleviated the progression of HCC by regulating miR-765. ${ }^{232}$ Therefore, endogenous or exogenous loading of beneficial ncRNAs into exosomes exhibits significant therapeutic potential.
Therapies that downregulate exosomal ncRNAs. It may also be therapeutic to decrease the amount of detrimental ncRNAs in exosomes. This effect can be achieved through endogenous or exogenous loading of small interfering RNAs (siRNAs) or ncRNA inhibitors such as antisense oligonucleotides (ASO) into exosomes. For instance, exosome-delivered circRNA has-circ-0005963 promotes drug resistance in CRC, whereas siRNA of has-circ-0005963 transported by exosomes can reverse resistance in vivo. ${ }^{281}$ Moreover, miR-21 is an oncogenic miRNA, while delivery of anti-miRNA oligonucleotides miR-21 using modified exosomes can reduce miR-21 level in the glioblastoma, contributing to reduction of tumor size. ${ }^{574}$

Above all, these studies provide new strategies for potential clinical diagnosis and therapeutic management for exosomemediated human diseases. Exosome-based delivery of curative ncRNAs, as well as siRNAs or inhibitors of harmful ncRNAs has the potential to be used in development of disease therapies. However, the transition of exosomal ncRNAs from basic laboratory research to clinical application remains challenging. Further development of biomedical materials and molecular targeted therapies is needed to elucidate the functions of exosomal ncRNAs.

\section{PERSPECTIVES AND CONCLUSIONS}

Knowledge of the roles of exosomal ncRNAs in physiological and pathological conditions has advanced considerably over the past few decades. In this review, we discuss the structures of exosomes and their cargo ncRNAs, and compile the underlying regulatory mechanisms. In order to distinguish between ncRNAs in exosomes and non-exosomes in health and disease, we also strive to study the differences in physiological homeostasis and pathological processes. Exosomes exert their roles in the pathophysiology of multiple disorders by modulating immune response, oxidative stress, autophagy, gut microbe, and cell cycle dysregulation. The impact of exosomes on cell biology is greater than originally expected, which makes related research rather complex. The temporal and spatial expression patterns, precise roles and mechanisms of specific miRNAs, IncRNAs, and circRNAs encapsulated in exosomes remain largely unknown in different system development and diseases. 
Interest in the contribution of exosomal ncRNAs to the progression of various diseases, including cancers, metabolic diseases, neurodegenerative diseases, cardiovascular diseases, autoimmune diseases, and infectious diseases, is booming. Tissue-specific alterations of exosomal ncRNAs play an important role in the initiation and development of human diseases as well as their complications. First, exosomal ncRNAs are involved in pathological cellular processes including cell proliferation, migration, invasion, metastasis, angiogenesis, and EMT associated with diverse cancers. Second, exosomal ncRNAs promote cell-to-cell and tissue-to-tissue crosstalk in an autocrine, paracrine or endocrine manner, and hence applying pleiotropic activities in metabolic diseases such as T2DM, obesity, and primary osteoporosis. Third, exosomal ncRNAs serve as regulators of dyslipidemia and myocardial structure and function in cardiovascular diseases including hypertension, AS, AMI, HF, and AF. Fourth, Exosomes carry ncRNAs to regulate communication between neurons and act as mediators involved in the pathogenesis and progression of neurodegenerative diseases. Fifth, exosomal ncRNAs modulate autoimmune diseases mainly by regulating inflammation and immune imbalance. Besides, the roles of exosomal ncRNAs in infectious diseases have been shown to be involved in the development of pneumonia and viral hepatitis. However, much effort remains to be done to understand the full extent of exosomal ncRNAs exerting their pathological effects.

Although exosomal ncRNAs have been identified for a relatively short time, there have been massive progression in clinical and therapeutic applications. In the field of diagnostics, it is noteworthy that exosomal ncRNAs are differentially expressed in human lifestyle activities and a variety of pathological diseases, suggesting that exosomal ncRNAs have the potential to be biomarkers for early detection. Exosomal ncRNAs exert advantages as biomarkers over non-exosomal ncRNAs. NcRNAs harbored in exosomes can be protected from degradation by RNase thus increasing their stability. Exosomal ncRNAs can present a high concentration in body fluid. ${ }^{575}$ Several studies have compared exosomal and non-exosomal miRNAs as biomarkers, and $75 \%$ of the studies reported that exosomal pattern of miRNAs are more important in biological processes compared with nonexosomal ones. This can be attributed to the advantages of exosomal miRNAs including quantity, quality, and stability. ${ }^{576}$ In the area of therapeutics, the use of exosomal ncRNAs have many potential benefits, so increasing exosomal ncRNAs therapies will enter a formal drug development process. Exosomes bearing a specific cargo can function as a drug delivery system. Exosomes have several advantages when delivering ncRNAs compared with traditional delivery methods, such as virus and liposome. Use of exosomes as endogenous vehicles can evade immune response, thus minimizing their toxicity. An in vivo study conducted by Mendt et al. reported no side effects including adverse immune response even after repeated administration of exosomes. ${ }^{570}$ Furthermore, exosomes can target specific tissues and cells through specific ligands on the surface. ${ }^{577}$ Moreover, exosomes can readily penetrate biological barriers such as the blood-brain barrier (BBB). For instance, an in vivo study reported that exosomes can cross the BBB and deliver siRNAs into the brain. ${ }^{571}$ Despite exosomal ncRNAs have great potential as biomarkers and therapeutics for a wide range of human diseases, there are many hurdles to bring them to clinic. First of all, we need to determine the extent to which purified exosomes are likely to be sufficient to confer the positive effects. Secondly, the dynamics and pharmacokinetics, as well as toxic studies of potential exosomal ncRNAs drugs require to be repeatedly tested. Besides, there is urgent need for experiments using animal and clinical models to identify whether exosomal ncRNAs exert regulatory homeostasis or pathological functions. Overcoming these obstacles will take the field to another unprecedented level.
The purpose of this study is to explore and strengthen the understanding of the mechanisms and roles of exosomal ncRNAs in human health and diseases, and to provide the basis for new clinical diagnosis and therapy strategies.

\section{ACKNOWLEDGEMENTS}

This work was supported by the National Natural Science Foundation of China (No. 82071593, 81770833, 81974223, and 82101663); the Fundamental Research Funds For the Central Universities of Central South University (NO. 2019zzts354).

\section{AUTHOR CONTRIBUTIONS}

C.L. and Y.-Q.N. wrote the manuscript and drew the figures. H.X., Q.-Y.X., and Y.Z. collected literature and summarized the table. J.-K.Z. supervised the manuscript and modified the figures. J.-Y.H. and S.L. polished language and corrected grammar errors. Y.-S.L. conceived the idea and directed the writing. All authors have read and approved the article.

\section{ADDITIONAL INFORMATION}

Competing interests: The authors declare no competing interests.

\section{REFERENCES}

1. Lakshmi, S., Hughes, T. A. \& Priya, S. Exosomes and exosomal RNAs in breast cancer: a status update. Eur. J. Cancer 144, 252-268 (2021).

2. Yang, D. et al. Progress, opportunity, and perspective on exosome isolation efforts for efficient exosome-based theranostics. Theranostics 10, 3684-3707 (2020).

3. Safdar, A., Saleem, A. \& Tarnopolsky, M. A. The potential of endurance exercisederived exosomes to treat metabolic diseases. Nat. Rev. Endocrinol. 12, 504-517 (2016).

4. Li, S. et al. Exosomes from hyperglycemia-stimulated vascular endothelial cells contain versican that regulate calcification/senescence in vascular smooth muscle cells. Cell Biosci. 9, 1 (2019).

5. Lin, X. et al. Exosomal Notch3 from high glucose-stimulated endothelial cells regulates vascular smooth muscle cells calcification/aging. Life Sci. 232, 116582 (2019).

6. Couzin, J. Cell biology: the ins and outs of exosomes. Science 308, 1862-1863 (2005).

7. Ni, Y.-Q., Lin, X., Zhan, J.-K. \& Liu, Y.-S. Roles and functions of exosomal noncoding RNAs in vascular aging. Aging Dis. 11, 164-178 (2020).

8. Jeppesen, D. K. et al. Reassessment of exosome composition. Cell 177, 428-445. e418 (2019)

9. Castaño, C., Novials, A. \& Párrizas, M. Exosomes and diabetes. Diabetes Metab. Res. Rev. 35, e3107 (2019).

10. Palade, G. E. A small particulate component of the cytoplasm. J. Biophys. Biochem Cytol. 1, 59-68 (1955).

11. Hoagland, M. B. et al. A soluble ribonucleic acid intermediate in protein synthesis. J. Biol. Chem. 231, 241-257 (1958).

12. Crick, F. H. On protein synthesis. Symp. Soc. Exp. Biol. 12, 138-163 (1958).

13. Tyc, K. \& Steitz, J. A. U3, U8 and U13 comprise a new class of mammalian snRNPs localized in the cell nucleolus. EMBO J. 8, 3113-3119 (1989).

14. Prestayko, A. W. \& Busch, H. Low molecular weight RNA of the chromatin fraction from Novikoff hepatoma and rat liver nuclei. Biochim. Biophys. Acta 169, 327-337 (1968)

15. Zieve, G. \& Penman, S. Small RNA species of the HeLa cell: metabolism and subcellular localization. Cell 8, 19-31 (1976).

16. Jarroux, J., Morillon, A. \& Pinskaya, M. History, discovery, and classification of IncRNAs. Adv. Exp. Med Biol. 1008, 1-46 (2017).

17. Sanger, H. L. et al. Viroids are single-stranded covalently closed circular RNA molecules existing as highly base-paired rod-like structures. Proc. Natl Acad. Sci. USA 73, 3852-3856 (1976)

18. Brannan, C. I., Dees, E. C., Ingram, R. S. \& Tilghman, S. M. The product of the H19 gene may function as an RNA. Mol. Cell Biol. 10, 28-36 (1990).

19. Lee, J. T., Davidow, L. S. \& Warshawsky, D. Tsix, a gene antisense to Xist at the X-inactivation centre. Nat. Genet 21, 400-404 (1999).

20. Brown, C. J. et al. A gene from the region of the human $X$ inactivation centre is expressed exclusively from the inactive $X$ chromosome. Nature 349, 38-44 (1991).

21. Lander, E. S. et al. Initial sequencing and analysis of the human genome. Nature 409, 860-921 (2001). 
22. The ENCODE Project Consortium. An integrated encyclopedia of DNA elements in the human genome. Nature. 489, 57-74, (2012).

23. Lee, R. C., Feinbaum, R. L. \& Ambros, V. The C. elegans heterochronic gene lin-4 encodes small RNAs with antisense complementarity to lin-14. Cell 75, 843-854 (1993).

24. Reinhart, B. J. et al. The 21-nucleotide let-7 RNA regulates developmental timing in Caenorhabditis elegans. Nature 403, 901-906 (2000).

25. Kapranov, P. et al. Large-scale transcriptional activity in chromosomes 21 and 22. Science 296, 916-919 (2002).

26. Okazaki, Y. et al. Analysis of the mouse transcriptome based on functional annotation of 60,770 full-length cDNAs. Nature 420, 563-573 (2002).

27. Rinn, J. L. et al. The transcriptional activity of human Chromosome 22. Genes Dev. 17, 529-540 (2003).

28. Kim, J. K. et al. Functional genomic analysis of RNA interference in C. elegans. Science 308, 1164-1167 (2005).

29. Bhat, A. A. et al. Role of non-coding RNA networks in leukemia progression, metastasis and drug resistance. Mol. Cancer 19, 57 (2020).

30. Anastasiadou, E., Jacob, L. S. \& Slack, F. J. Non-coding RNA networks in cancer. Nat. Rev. Cancer 18, 5-18 (2018).

31. Valadi, $\mathrm{H}$. et al. Exosome-mediated transfer of mRNAs and microRNAs is a novel mechanism of genetic exchange between cells. Nat. Cell Biol. 9, 654-659 (2007).

32. Huang, X. et al. Characterization of human plasma-derived exosomal RNAs by deep sequencing. BMC Genomics 14, 319 (2013).

33. van Balkom, B. W. et al. Quantitative and qualitative analysis of small RNAs in human endothelial cells and exosomes provides insights into localized RNA processing, degradation and sorting. J. Extracell. Vesicles 4, 26760 (2015).

34. Mori, M. A. et al. Extracellular miRNAs: from biomarkers to mediators of physiology and disease. Cell Metab. 30, 656-673 (2019).

35. Zhang, J. et al. Exosome and exosomal microRNA: trafficking, sorting, and function. Genomics Proteom. Bioinforma. 13, 17-24 (2015).

36. Shan, S.-K. et al. Exosomes and bone disease. Curr. Pharm. Des. 25, 4536-4549 (2020)

37. Kita, S., Maeda, N. \& Shimomura, I. Interorgan communication by exosomes, adipose tissue, and adiponectin in metabolic syndrome. J. Clin. Investig. 129, 4041-4049 (2019).

38. Kamerkar, S. et al. Exosomes facilitate therapeutic targeting of oncogenic KRAS in pancreatic cancer. Nature 546, 498-503 (2017).

39. Lei, Y. T., Liu, X. G. \& Xu, S. Exosomal CircRNAs in Aging and Aging-related Diseases. Chin. J. Biochem. Mol. Biol. https://doi.org/10.13865/j.cnki.cjbmb.2021.03.1621 (2021).

40. Su, T. et al. Bone marrow mesenchymal stem cells-derived exosomal MiR-29b-3p regulates aging-associated insulin resistance. ACS Nano 13, 2450-2462 (2019).

41. Prattichizzo, F. et al. Exosome-based immunomodulation during aging: A nanoperspective on inflamm-aging. Mech. Ageing Dev. 168, 44-53 (2017).

42. Sun, Y. et al. Expression of miRNA-29 in Pancreatic $\beta$ Cells Promotes Inflammation and Diabetes via TRAF3. Cell Rep. 34, 108576 (2021).

43. Kamalden, T. A. et al. Exosomal MicroRNA-15a transfer from the pancreas augments diabetic complications by inducing oxidative stress. Antioxid. Redox Signal. 27, 913-930 (2017).

44. He, Q. et al. Mesenchymal stem cell-derived exosomes exert ameliorative effects in type 2 diabetes by improving hepatic glucose and lipid metabolism via enhancing autophagy. Stem Cell Res. Ther. 11, 223 (2020).

45. Chelakkot, C. et al. Akkermansia muciniphila-derived extracellular vesicles influence gut permeability through the regulation of tight junctions. Exp. Mol. Med. 50, e450 (2018).

46. Gehrmann, U. et al. Harnessing the exosome-induced immune response for cancer immunotherapy. Semin Cancer Biol. 28, 58-67 (2014).

47. Kurywchak, P., Tavormina, J. \& Kalluri, R. The emerging roles of exosomes in the modulation of immune responses in cancer. Genome Med 10, 23 (2018).

48. Kalluri, R. \& LeBleu, V. S. The biology, function, and biomedical applications of exosomes. Science 7, 367 (2020).

49. Li, W., Jin, L.-Y., Cui, Y.-B. \& Xie, N. Human umbilical cord mesenchymal stem cells-derived exosomal microRNA-17-3p ameliorates inflammatory reaction and antioxidant injury of mice with diabetic retinopathy via targeting STAT1. Int. Immunopharmacol. 90, 107010 (2021).

50. Venkat, P. et al. CD133+ exosome treatment improves cardiac function after stroke in type 2 diabetic mice. Transl. stroke Res. 12, 112-124 (2021).

51. Govindappa, P. K. et al. Targeting exosome-associated human antigen R attenuates fibrosis and inflammation in diabetic heart. FASEB J.: 34, 2238-2251 (2020).

52. Li, W. et al. Bone marrow mesenchymal stem cells-induced exosomal microRNA486-3p protects against diabetic retinopathy through TLR4/NF-?B axis repression. J. Endocrinol. Invest. 44, 1193-1207 (2020).

53. Gu, C., Zhang, H. \& Gao, Y. Adipose mesenchymal stem cells-secreted extracellular vesicles containing microRNA-192 delays diabetic retinopathy by targeting ITGA1. J. Cell. Physiol. 236, 5036-5051 (2020).
54. Cui, X. et al. Circulating exosomes activate dendritic cells and induce unbalanced CD4+ T cell differentiation in hashimoto thyroiditis. J. Clin. Endocrinol. Metab. 104, 4607-4618 (2019).

55. Zitvogel, L. et al. Eradication of established murine tumors using a novel cellfree vaccine: dendritic cell-derived exosomes. Nat. Med. 4, 594-600 (1998).

56. Cheng, Y. \& Schorey, J. S. Exosomes carrying mycobacterial antigens can protect mice against Mycobacterium tuberculosis infection. Eur. J. Immunol. 43, 3279-3290 (2013).

57. Ding, G. et al. Pancreatic cancer-derived exosomes transfer miRNAs to dendritic cells and inhibit RFXAP expression via miR-212-3p. Oncotarget 6, 29877-29888 (2015).

58. Pegtel, D. M. et al. Functional delivery of viral miRNAs via exosomes. Proc. Natl Acad. Sci. USA 107, 6328-6333 (2010).

59. Dini, L. et al. Microvesicles and exosomes in metabolic diseases and inflammation. Cytokine Growth Factor Rev. 51, 27-39 (2020).

60. Kranendonk, M. E. G. et al. Human adipocyte extracellular vesicles in reciprocal signaling between adipocytes and macrophages. Obes. (Silver Spring, Md.) 22, 1296-1308 (2014).

61. Hirsova, P. et al. Lipid-induced signaling causes release of inflammatory extracellular vesicles from hepatocytes. Gastroenterology 150, 956-967 (2016).

62. Song, M. et al. Adipocyte-derived exosomes carrying sonic hedgehog mediate M1 macrophage polarization-induced insulin resistance via Ptch and PI3K pathways. Cell. Physiol. Biochem. 48, 1416-1432 (2018).

63. Deng, Z.-b et al. Adipose tissue exosome-like vesicles mediate activation of macrophage-induced insulin resistance. Diabetes 58, 2498-2505 (2009).

64. Xie, Z. et al. Adipose-derived exosomes exert proatherogenic effects by regulating macrophage foam cell formation and polarization.J. Am. Heart Assoc 7, e007442 (2018).

65. Xiao, M., Zhang, J., Chen, W. \& Chen, W. M1-like tumor-associated macrophages activated by exosome-transferred THBS1 promote malignant migration in oral squamous cell carcinoma. J. Exp. Clin. Cancer Res. 37, 143 (2018).

66. Zhao, H. et al. Exosomes from adipose-derived stem cells attenuate adipose inflammation and obesity through polarizing $\mathrm{M} 2$ Macrophages and beiging in white adipose tissue. Diabetes 67, 235-247 (2018).

67. Li, J. et al. Exosomes derived from mesenchymal stem cells attenuate the progression of atherosclerosis in $\mathrm{ApoE}(-/-)$ mice via miR-let7 mediated infiltration and polarization of M2 macrophage. Biochem. Biophys. Res. Commun. 510, 565-572 (2019).

68. Zhao, J. et al. Mesenchymal stromal cell-derived exosomes attenuate myocardial ischaemia-reperfusion injury through miR-182-regulated macrophage polarization. Cardiovasc Res. 115, 1205-1216 (2019).

69. Deng, S. et al. Exosomes from adipose-derived mesenchymal stem cells ameliorate cardiac damage after myocardial infarction by activating S1P/SK1/ S1PR1 signaling and promoting macrophage M2 polarization. Int. J. Biochem Cell Biol. 114, 105564 (2019).

70. López-Janeiro, Á. et al. Prognostic value of macrophage polarization markers in epithelial neoplasms and melanoma. A systematic review and meta-analysis. Mod. Pathol. 33, 1458-1465 (2020).

71. Wang, L. P. et al. Exosomal DLX6-AS1 from hepatocellular carcinoma cells induces M2 macrophage polarization to promote migration and invasion in hepatocellular carcinoma through microRNA-15a-5p/CXCL17 axis. J. Exp. Clin. Cancer Res. 40, 177 (2021).

72. Zhang, X. et al. Tumor-derived exosomes induce N2 polarization of neutrophils to promote gastric cancer cell migration. Mol. Cancer 17, 146 (2018).

73. Jiang, M. et al. Keratinocyte exosomes activate neutrophils and enhance skin inflammation in psoriasis. FASEB J. 33, 13241-13253 (2019).

74. Piccard, H., Muschel, R. J. \& Opdenakker, G. On the dual roles and polarized phenotypes of neutrophils in tumor development and progression. Crit. Rev. Oncol. Hematol. 82, 296-309 (2012).

75. Yao, J. et al. Extracellular vesicles derived from human umbilical cord mesenchymal stem cells alleviate rat hepatic ischemia-reperfusion injury by suppressing oxidative stress and neutrophil inflammatory response. FASEB J. 33, 1695-1710 (2019).

76. Liu, J. et al. Clinical Potential of Extracellular Vesicles in Type 2 Diabetes. Front. Endocrinol. 11, 596811 (2020).

77. Silva, N. D., Samblas, M., Martínez, J. A. \& Milagro, F. I. Effects of exosomes from LPS-activated macrophages on adipocyte gene expression, differentiation, and insulin-dependent glucose uptake. J. Physiol. Biochem. 74, 559-568 (2018)

78. Castaño, C., Kalko, S., Novials, A. \& Párrizas, M. Obesity-associated exosomal miRNAs modulate glucose and lipid metabolism in mice. Proc. Natl Acad. Sci. USA 115, 12158-12163 (2018).

79. Ding, $X$. et al. MiR-21-5p in macrophage-derived extracellular vesicles affects podocyte pyroptosis in diabetic nephropathy by regulating A20. J. Endocrinol. Invest. 44, 1175-1184 (2020). 
80. Fabbri, M. et al. MicroRNAs bind to Toll-like receptors to induce prometastatic inflammatory response. Proc. Natl Acad. Sci. USA 109, E2110-E2116 (2012).

81. Shi, X. et al. Paeonol inhibits NLRP3 mediated inflammation in rat endothelial cells by elevating hyperlipidemic rats plasma exosomal miRNA-223. Eur.J, Pharmacol. 885, 173473 (2020).

82. Valencia, K. et al. miRNA cargo within exosome-like vesicle transfer influences metastatic bone colonization. Mol. Oncol. 8, 689-703 (2014).

83. Rani, V. et al. Oxidative stress and metabolic disorders: pathogenesis and therapeutic strategies. Life Sci. 148, 183-193 (2016).

84. Zhang, C. et al. Exosomes, the message transporters in vascular calcification. J. Cell. Mol. Med. 22, 4024-4033 (2018).

85. Wen, Z. et al. Hypertrophic adipocyte-derived exosomal miR-802-5p contributes to insulin resistance in cardiac myocytes through targeting HSP60. Obes. (Silver Spring, Md.). 28, 1932-1940 (2020).

86. Varela-López, A. et al. Age-related loss in bone mineral density of rats fed lifelong on a fish oil-based diet is avoided by coenzyme Q10 addition. Nutrients. 9, 176 (2017).

87. Xie, Y. et al. Involvement of serum-derived exosomes of elderly patients with bone loss in failure of bone remodeling via alteration of exosomal bone-related proteins. Aging Cell 17, e12758 (2018).

88. Wang, Y. et al. Exosomal circHIPK3 released from hypoxia-pretreated cardiomyocytes regulates oxidative damage in cardiac microvascular endothelial cells via the miR-29a/IGF-1 Pathway. Oxid. Med Cell Longev. 2019, 7954657 (2019).

89. Xu, J., Camfield, R. \& Gorski, S. M. The interplay between exosomes and autophagy - partners in crime. J. Cell Sci. 131, jcs215210 (2018).

90. Wang, F., Jia, J. \& Rodrigues, B. Autophagy, metabolic disease, and pathogenesis of heart dysfunction. Can J. Cardiol. 33, 850-859 (2017).

91. Xu, J., Camfield, R. \& Gorski, S. M. The interplay between exosomes and autophagy - partners in crime. J. Cell Sci. 131, jcs215210 (2018).

92. Villarroya-Beltri, C. et al. ISGylation controls exosome secretion by promoting lysosomal degradation of MVB proteins. Nat. Commun. 7, 13588 (2016).

93. Kumar, D., Gupta, D., Shankar, S. \& Srivastava, R. K. Biomolecular characterization of exosomes released from cancer stem cells: Possible implications for biomarker and treatment of cancer. Oncotarget 6, 3280-3291 (2015).

94. Qi, M. et al. Autophagy maintains the function of bone marrow mesenchymal stem cells to prevent estrogen deficiency-induced osteoporosis. Theranostics 7, 4498-4516 (2017).

95. Liu, H. et al. Intermittent fasting preserves beta-cell mass in obesity-induced diabetes via the autophagy-lysosome pathway. Autophagy 13, 1952-1968 (2017).

96. Yang, L. et al. Defective hepatic autophagy in obesity promotes ER stress and causes insulin resistance. Cell Metab. 11, 467-478 (2010).

97. Li, F. et al. Adipose-specific knockdown of Sirt1 results in obesity and insulin resistance by promoting exosomes release. Cell Cycle (Georget., Tex.) 18, 2067-2082 (2019).

98. Hu, J. et al. Exosomal Mst1 transfer from cardiac microvascular endothelial cells to cardiomyocytes deteriorates diabetic cardiomyopathy. Biochim. Biophys. Acta Mol. Basis Dis. 1864, 3639-3649 (2018).

99. Shi, R. et al. Exosomes derived from mmu_circ_0000250-modified adiposederived mesenchymal stem cells promote wound healing in diabetic mice by inducing miR-128-3p/SIRT1-mediated autophagy. Am. J. Physiol. Cell Physiol. 318, C848-C856 (2020).

100. Jin, J. et al. Exosome secreted from adipose-derived stem cells attenuates diabetic nephropathy by promoting autophagy flux and inhibiting apoptosis in podocyte. Stem Cell Res. Ther. 10, 95 (2019).

101. Ebrahim, N. et al. Mesenchymal stem cell-derived exosomes ameliorated diabetic nephropathy by autophagy induction through the mTOR signaling pathway. Cells. 7, (2018).

102. Huang, $H$. et al. $M 2$ macrophage-derived exosomal miR-25-3p improves high glucose-induced podocytes injury through activation autophagy via inhibiting DUSP1 expression. IUBMB life. 72, 2651-2662 (2020).

103. Yang, Y. et al. Secretory carrier membrane protein 5 is an autophagy inhibitor that promotes the secretion of a-synuclein via exosome. PLOS ONE 12, e0180892 (2017).

104. Wang, B. et al. Exosomal miR-1910-3p promotes proliferation, metastasis, and autophagy of breast cancer cells by targeting MTMR3 and activating the NF-KB signaling pathway. Cancer Lett. 489, 87-99 (2020).

105. Colletti, M., Ceglie, D., Di Giannatale, A. \& Nazio, F. Autophagy and exosomes relationship in cancer: friends or foes? Front. Cell Dev. Biol. 8, 614178 (2020).

106. Yao, W., Guo, P., Mu, Q. \& Wang, Y. Exosome-derived Circ-PVT1 contributes to cisplatin resistance by regulating autophagy, invasion, and apoptosis via miR30a-5p/YAP1 axis in gastric cancer cells. Cancer Biother. Radiopharm. 36, 347-359 (2021).

107. Zhang, Y. J. et al. Impacts of gut bacteria on human health and diseases. Int J. Mol. Sci. 16, 7493-7519 (2015)
108. Fan, Y. \& Pedersen, O. Gut microbiota in human metabolic health and disease Nat. Rev. Microbiol. 19, 55-71 (2021).

109. Baothman, O. A. et al. The role of gut microbiota in the development of obesity and Diabetes. Lipids Health Dis. 15, 108 (2016).

110. Gopalakrishnan, V. et al. The influence of the gut microbiome on cancer immunity, and cancer immunotherapy. Cancer Cell 33, 570-580 (2018).

111. Liu, S. et al. The host shapes the gut microbiota via fecal microRNA. Cell host microbe 19, 32-43 (2016).

112. Ashrafian, F. et al. Akkermansia muciniphila-derived extracellular vesicles as a mucosal delivery vector for amelioration of obesity in mice. Front. Microbiol. 10, 2155 (2019).

113. Choi, Y. et al. Gut microbe-derived extracellular vesicles induce insulin resistance, thereby impairing glucose metabolism in skeletal muscle. Sci. Rep. 5, 15878 (2015).

114. Luo, Z. et al. CRlg+ Macrophages prevent gut microbial DNA-containing extracellular vesicle-induced tissue inflammation and insulin resistance. Gastroenterology 160, 863-874 (2021).

115. Liu, J. H. et al. Extracellular vesicles from child gut microbiota enter into bone to preserve bone mass and strength. Adv. Sci. 8, 2004831 (2021).

116. Williams, G. H. \& Stoeber, K. The cell cycle and cancer. J. Pathol. 226, 352-364 (2012).

117. Dai, X. et al. Exosomal circRNA 100284 from arsenite-transformed cells, via microRNA-217 regulation of EZH2, is involved in the malignant transformation of human hepatic cells by accelerating the cell cycle and promoting cell proliferation. Cell Death Dis. 9, 454 (2018).

118. Pan, L. et al. Exosomes-mediated transfer of long noncoding RNA ZFAS1 promotes gastric cancer progression. J. Cancer Res Clin. Oncol. 143, 991-1004 (2017).

119. Reza, A., Choi, Y. J., Yasuda, H. \& Kim, J. H. Human adipose mesenchymal stem cell-derived exosomal-miRNAs are critical factors for inducing anti-proliferation signalling to A2780 and SKOV-3 ovarian cancer cells. Sci. Rep. 6, 38498 (2016).

120. Arroyo, J. D. et al. Argonaute 2 complexes carry a population of circulating microRNAs independent of vesicles in human plasma. Proc. Natl Acad. Sci. USA 108, 5003-5008 (2011).

121. Wang, K. et al. Export of microRNAs and microRNA-protective protein by mammalian cells. Nucleic Acids Res. 38, 7248-7259 (2010).

122. Tosar, J. P., Witwer, K. \& Cayota, A. Revisiting extracellular RNA release, processing, and function. Trends Biochem Sci. 46, 438-445 (2021).

123. Vickers, K. C. et al. MicroRNAs are transported in plasma and delivered to recipient cells by high-density lipoproteins. Nat. Cell Biol. 13, 423-433 (2011).

124. Tosar, J. P. et al. Fragmentation of extracellular ribosomes and tRNAs shapes the extracellular RNAome. Nucleic Acids Res. 48, 12874-12888 (2020).

125. Nechooshtan, G., Yunusov, D., Chang, K. \& Gingeras, T. R. Processing by RNase 1 forms tRNA halves and distinct $Y$ RNA fragments in the extracellular environment. Nucleic Acids Res. 48, 8035-8049 (2020).

126. Martínez, M. C. \& Andriantsitohaina, R. Extracellular vesicles in metabolic syndrome. Circulation Res. 120, 1674-1686 (2017).

127. Muraca, M. \& Cappariello, A. The role of extracellular vesicles (EVs) in the epigenetic regulation of bone metabolism and osteoporosis. Int. J. Mol. Sci. 21 8682 (2020).

128. Prattichizzo, F. et al. Extracellular vesicle-shuttled miRNAs: a critical appraisal of their potential as nano-diagnostics and nano-therapeutics in type 2 diabetes mellitus and its cardiovascular complications. Theranostics 11, 1031-1045 (2021).

129. $\mathrm{Wu}, \mathrm{P}$. et al. Emerging role of tumor-related functional peptides encoded by IncRNA and circRNA. Mol. cancer 19, 22 (2020).

130. Torre, L. A. et al. Global cancer statistics, 2012. CA: Cancer J. Clin. 65, 87-108 (2015).

131. Xie, Y. et al. The role of exosomal noncoding RNAs in cancer. Mol. cancer 18, 37 (2019).

132. $\mathrm{Hu}, \mathrm{C}$. et al. Role of exosomal microRNAs in lung cancer biology and clinical applications. Cell Prolif. 53, e12828 (2020).

133. He, Y. et al. Exosomal microRNA: a novel biomarker for breast cancer. Biomark Med. 12, 177-188 (2018)

134. Li, S. et al. Exosomal miRNAs in hepatocellular carcinoma development and clinical responses. J. Hematol. Oncol. 11, 54 (2018).

135. Molina, J. R. et al. Non-small cell lung cancer: epidemiology, risk factors, treatment, and survivorship. Mayo Clin. Proc. 83, 584-594 (2008).

136. Hanahan, D. \& Weinberg, R. A. Hallmarks of cancer: the next generation. Cell 144, 646-674 (2011).

137. Kim, J. et al. Exosome cargo reflects TGF- $\beta 1$-mediated epithelial-tomesenchymal transition (EMT) status in A549 human lung adenocarcinoma cells. Biochem. Biophys. Res. Commun. 478, 643-648 (2016).

138. Zhang, X. et al. Hypoxic BMSC-derived exosomal miRNAs promote metastasis of lung cancer cells via STAT3-induced EMT. Mol. Cancer 18, 40 (2019). 
139. He, S. et al. Exosomal miR-499a-5p promotes cell proliferation, migration and EMT via mTOR signaling pathway in lung adenocarcinoma. Exp. Cell Res. 379, 203-213 (2019).

140. Patterson, J. W. Recent advances in dermatopathology. Va. Med. 115, 572-577 (1988).

141. $\mathrm{Wu}, \mathrm{H}$. et al. Circulating exosomal microRNA-96 promotes cell proliferation, migration and drug resistance by targeting LMO7. J. Cell. Mol. Med. 21, 1228-1236 (2017).

142. Cheng, G. et al. Targeting lonidamine to mitochondria mitigates lung tumorigenesis and brain metastasis. Nat. Commun. 10, 2205 (2019).

143. Wei, F. et al. Correction to: Exosomes derived from gemcitabine resistant cells transfer malignant phenotypic traits via delivery of miRNA-222-3p. Mol. Cancer 20, 35 (2021).

144. Grimolizzi, F. et al. Exosomal miR-126 as a circulating biomarker in non-small-cell lung cancer regulating cancer progression. Sci. Rep. 7, 15277 (2017).

145. Tang, Y. et al. Erratum to: Radiation-induced miR-208a increases the proliferation and radioresistance by targeting p21 in human lung cancer cells. J. Exp. Clin. Cancer Res. 35, 20 (2016).

146. Adi Harel, S. et al. Reactivation of epigenetically silenced miR-512 and miR-373 sensitizes lung cancer cells to cisplatin and restricts tumor growth. Cell Death Differ. 22, 1328-1340 (2015).

147. Folkman, J. Role of angiogenesis in tumor growth and metastasis. Semin. Oncol. 29, 15-18 (2002).

148. Zhuang, G. et al. Tumour-secreted miR-9 promotes endothelial cell migration and angiogenesis by activating the JAK-STAT pathway. EMBO J. 31, 3513-3523 (2012).

149. Liu, Y. et al. STAT3-regulated exosomal miR-21 promotes angiogenesis and is involved in neoplastic processes of transformed human bronchial epithelial cells. Cancer Lett. 370, 125-135 (2016).

150. Hsu, Y. L. et al. Hypoxic lung cancer-secreted exosomal miR-23a increased angiogenesis and vascular permeability by targeting prolyl hydroxylase and tight junction protein ZO-1. Oncogene 36, 4929-4942 (2017).

151. Cui, H. et al. Tissue inhibitor of metalloproteinases-1 induces a pro-tumourigenic increase of miR-210 in lung adenocarcinoma cells and their exosomes. Oncogene 34, 3640-3650 (2015).

152. $\mathrm{Xu}$, Z. et al. Lung adenocarcinoma cell-derived exosomal miR-21 facilitates osteoclastogenesis. Gene 666, 116-122 (2018).

153. Poulet, C. et al. Exosomal long non-coding RNAs in lung diseases. Int. J. Mol. Sci. 21, 3580 (2020).

154. Li, C. et al. Tumor-derived exosomal IncRNA GAS5 as a biomarker for early-stage non-small-cell lung cancer diagnosis. J. Cell. Physiol. 234, 20721-20727 (2019).

155. Teng, Y., Kang, H. \& Chu, Y. Identification of an exosomal long noncoding RNA SOX2-OT in plasma as a promising biomarker for lung squamous cell carcinoma. Genet. Test. Mol. Biomark. 23, 235-240 (2019).

156. Zhang, N. et al. Circular RNA circSATB2 promotes progression of non-small cell lung cancer cells. Mol. cancer 19, 101 (2020).

157. Xu, X., Tao, R., Sun, L. \& Ji, X. Exosome-transferred hsa_circ_0014235 promotes DDP chemoresistance and deteriorates the development of non-small cell lung cancer by mediating the miR-520a-5p/CDK4 pathway. Cancer Cell Int. 20, 552 (2020).

158. Wang, J. et al. circRNA-002178 act as a ceRNA to promote PDL1/PD1 expression in lung adenocarcinoma. Cell Death Dis. 11, 32 (2020).

159. Xian, J. et al. Identification of three circular RNA cargoes in serum exosomes as diagnostic biomarkers of non-small-cell lung cancer in the Chinese population. J. Mol. Diagnostics 22, 1096-1108 (2020).

160. Shi, Q. et al. Serum exosomes-based biomarker circ_0008928 Regulates cisplatin sensitivity, tumor progression, and glycolysis metabolism by miR-488/HK2 axis in cisplatin-resistant nonsmall cell lung carcinoma. Cancer Biother. Radiopharm. https://doi.org/10.1089/cbr.2020.4490 (2021).

161. Yang, B. et al. Tumor-derived exosomal circRNA_102481 contributes to EGFRTKIs resistance via the miR-30a-5p/ROR1 axis in non-small cell lung cancer. Aging 13, 13264-13286 (2021).

162. Ding, C. et al. Exosomal Circ-MEMO1 promotes the progression and aerobic glycolysis of non-small cell lung cancer through targeting MiR-101-3p/KRAS axis. Front. Genet. 11, 962 (2020).

163. Fang, K. et al. Serum-derived exosomes-mediated circular RNA ARHGAP10 modulates the progression of non-small-cell lung cancer through the miR-638/ FAM83F Axis. Cancer Biother. Radiopharm. https://doi.org/10.1089/cbr.2019.3534 (2020).

164. Shao, N., Song, L. \& Sun, X. Exosomal circ_PIP5K1A regulates the progression of non-small cell lung cancer and cisplatin sensitivity by miR-101/ABCC1 axis. Mol. Cell Biochem 476, 2253-2267 (2021).

165. Chen, T. et al. Tumor-derived exosomal circFARSA mediates M2 macrophage polarization via the PTEN/PI3K/AKT pathway to promote non-small cell lung cancer metastasis. Cancer Treat. Res. Commun. 28, 100412 (2021).
166. Chen, F. et al. Circular RNAs expression profiles in plasma exosomes from earlystage lung adenocarcinoma and the potential biomarkers. J. Cell. Biochem. 121, 2525-2533 (2020).

167. Harbeck, N. et al. Breast cancer. Nat. Rev. Dis. Prim. 5, 66 (2019).

168. Bray, F. et al. Global cancer statistics 2018: GLOBOCAN estimates of incidence and mortality worldwide for 36 cancers in 185 countries. CA: Cancer J. Clin. 68, 394-424 (2018).

169. Liu, Q., Peng, F. \& Chen, J. The role of exosomal MicroRNAs in the tumor microenvironment of breast cancer. Int. J. Mol. Sci. 20, 3884 (2019).

170. Singh, R. et al. Exosome-mediated transfer of miR-10b promotes cell invasion in breast cancer. Mol. cancer 13, 256 (2014).

171. Li, X. J., Ren, Z. J., Tang, J. H. \& Yu, Q. Exosomal microRNA MiR-1246 promotes cell proliferation, invasion and drug resistance by targeting CCNG2 in breast cancer. Cell. Physiol. Biochem. 44, 1741-1748 (2017).

172. O'Brien, K. et al. miR-134 in extracellular vesicles reduces triple-negative breast cancer aggression and increases drug sensitivity. Oncotarget 6, 32774-32789 (2015).

173. Kong, X. et al. MiR-130a-3p inhibits migration and invasion by regulating RAB5B in human breast cancer stem cell-like cells. Biochem. Biophys. Res. Commun. 501, 486-493 (2018).

174. Baroni, S. et al. Exosome-mediated delivery of miR-9 induces cancer-associated fibroblast-like properties in human breast fibroblasts. Cell death Dis. 7, e2312 (2016).

175. Wu, H. J., Hao, M., Yeo, S. K. \& Guan, J. L. FAK signaling in cancer-associated fibroblasts promotes breast cancer cell migration and metastasis by exosomal miRNAs-mediated intercellular communication. Oncogene 39, 2539-2549 (2020).

176. Donnarumma, E. et al. Cancer-associated fibroblasts release exosomal microRNAs that dictate an aggressive phenotype in breast cancer. Oncotarget 8 , 19592-19608 (2017).

177. Kim, K. S. et al. ELK3 expressed in lymphatic endothelial cells promotes breast cancer progression and metastasis through exosomal miRNAs. Sci. Rep. 9, 8418 (2019).

178. Zhou, W. et al. Cancer-secreted miR-105 destroys vascular endothelial barriers to promote metastasis. Cancer cell 25, 501-515 (2014).

179. Fong, M. Y. et al. Breast-cancer-secreted miR-122 reprograms glucose metabolism in premetastatic niche to promote metastasis. Nat. cell Biol. 17, 183-194 (2015).

180. Le, M. T. et al. miR-200-containing extracellular vesicles promote breast cancer cell metastasis. J. Clin. Investig. 124, 5109-5128 (2014).

181. Di Modica, M. et al. Breast cancer-secreted miR-939 downregulates VE-cadherin and destroys the barrier function of endothelial monolayers. Cancer Lett. $\mathbf{3 8 4}$, 94-100 (2017).

182. Hashimoto, K. et al. Cancer-secreted hsa-miR-940 induces an osteoblastic phenotype in the bone metastatic microenvironment via targeting ARHGAP1 and FAM134A. Proc. Natl Acad. Sci. USA 115, 2204-2209 (2018).

183. Guo, L. et al. Breast cancer cell-derived exosomal miR-20a-5p promotes the proliferation and differentiation of osteoclasts by targeting SRCIN1. Cancer Med. 8, 5687-5701 (2019).

184. Ono, M. et al. Exosomes from bone marrow mesenchymal stem cells contain a microRNA that promotes dormancy in metastatic breast cancer cells. Sci. Signal. 7, ra63 (2014).

185. Kosaka, N. et al. Neutral sphingomyelinase 2 (nSMase2)-dependent exosomal transfer of angiogenic microRNAs regulate cancer cell metastasis. J. Biol. Chem. 288, 10849-10859 (2013)

186. Lee, J. K. et al. Exosomes derived from mesenchymal stem cells suppress angiogenesis by down-regulating VEGF expression in breast cancer cells. PLoS ONE 8, e84256 (2013).

187. Pakravan, K. et al. MicroRNA-100 shuttled by mesenchymal stem cell-derived exosomes suppresses in vitro angiogenesis through modulating the mTOR/HIF1a/VEGF signaling axis in breast cancer cells. Cell. Oncol. (Dordr.) 40, 457-470 (2017).

188. Zhang, P. et al. Exosome-mediated delivery of MALAT1 induces cell proliferation in breast cancer. OncoTargets Ther. 11, 291-299 (2018).

189. Lu, Y., Chen, L., Li, L. \& Cao, Y. Exosomes derived from brain metastatic breast cancer cells destroy the blood-brain barrier by carrying IncRNA GS1-600G8.5. Biomed. Res. Int. 2020, 7461727 (2020).

190. Zhong, G. et al. Determination of serum exosomal H19 as a noninvasive biomarker for breast cancer diagnosis. OncoTargets Ther. 13, 2563-2571 (2020).

191. Na-Er, A., Xu, Y. Y., Liu, Y. H. \& Gan, Y. J. Upregulation of serum exosomal SUMO1P3 predicts unfavorable prognosis in triple negative breast cancer. Eur. Rev. Med. Pharmacol. Sci. 25, 154-160 (2021).

192. Lan, F. et al. Serum exosomal IncRNA XIST is a potential non-invasive biomarker to diagnose recurrence of triple-negative breast cancer. J. Cell. Mole. Med. 25, $7602-7607$ (2021). 
193. Tang, S. et al. Overexpression of serum exosomal HOTAIR is correlated with poor survival and poor response to chemotherapy in breast cancer patients. J. Biosci. 44, 37 (2019).

194. Wang, Y. L. et al. Long non-coding RNA HOTAIR in circulatory exosomes is correlated with ErbB2/HER2 positivity in breast cancer. Breast (Edinb., Scotl.) 46, 64-69 (2019).

195. Yang, S. J. et al. Tumor-derived exosomal circPSMA1 facilitates the tumorigenesis, metastasis, and migration in triple-negative breast cancer (TNBC) through miR-637/Akt1/ $\beta$-catenin (cyclin D1) axis. Cell Death Dis. 12, 420 (2021).

196. Zhan, Y. et al. Carcinoma-associated fibroblasts derived exosomes modulate breast cancer cell stemness through exonic circHIF1A by miR-580-5p in hypoxic stress. Cell Death Discov. 7, 141 (2021).

197. Chen, T. et al. CircHIF1A regulated by FUS accelerates triple-negative breast cancer progression by modulating NFIB expression and translocation. Oncogene 40, 2756-2771 (2021)

198. Wang, J. et al. Circular RNA expression in exosomes derived from breast cancer cells and patients. Epigenomics 11, 411-421 (2019).

199. Forner, A., Llovet, J. M. \& Bruix, J. Hepatocellular carcinoma. Lancet (Lond., Engl.) 379, 1245-1255 (2012).

200. Forner, A., Reig, M. \& Bruix, J. Hepatocellular carcinoma. Lancet (Lond., Engl.) 391 1301-1314 (2018).

201. Sorop, A. et al. Exosomal microRNAs as biomarkers and therapeutic targets for hepatocellular carcinoma. Int. J. Mol. Sci. 22, 4997 (2021).

202. Cui, Y. et al. Mechanism of exosomal microRNA-224 in development of hepatocellular carcinoma and its diagnostic and prognostic value. World J. Gastroenterol. 25, 1890-1898 (2019).

203. Xue, X. et al. Exosomal miR-93 promotes proliferation and invasion in hepatocellular carcinoma by directly inhibiting TIMP2/TP53INP1/CDKN1A. Biochem. Biophys. Res. Commun. 502, 515-521 (2018).

204. Qu, Z. et al. Exosomal miR-665 as a novel minimally invasive biomarker for hepatocellular carcinoma diagnosis and prognosis. Oncotarget 8, 80666-80678 (2017).

205. Fang, T. et al. Tumor-derived exosomal miR-1247-3p induces cancer-associated fibroblast activation to foster lung metastasis of liver cancer. Nat. Commun. 9 , 191 (2018).

206. Lin, X. J. et al. Hepatocellular carcinoma cell-secreted exosomal microRNA-210 promotes angiogenesis in vitro and in vivo. Mol. Ther. Nucleic Acids 11, 243-252 (2018).

207. Matsuura, Y. et al. Exosomal miR-155 Derived from hepatocellular carcinoma cells under hypoxia promotes angiogenesis in endothelial cells. Digestive Dis. Sci. 64, 792-802 (2019).

208. Lee, Y. R. et al. Circulating exosomal noncoding RNAs as prognostic biomarkers in human hepatocellular carcinoma. Int. J. cancer 144, 1444-1452 (2019).

209. Wang, $\mathrm{H}$. et al. Expression of serum exosomal microRNA-21 in human hepatocellular carcinoma. Biomed. Res. Int 2014, 864894 (2014).

210. Nakano, T. et al. Circulating exosomal miR-92b: Its role for cancer immunoediting and clinical value for prediction of posttransplant hepatocellular carcinoma recurrence. Am. J. Transplant. 19, 3250-3262 (2019).

211. Cho, H. J. et al. Serum exosomal MicroRNA, miR-10b-5p, as a potential diagnostic biomarker for early-stage hepatocellular carcinoma. J. Clin. Med. 9, 281 (2020).

212. Matboli, M. et al. Exosomal miR-1298 and IncRNA-RP11-583F2.2 Expression in Hepato-cellular Carcinoma. Curr. genomics 21, 46-55 (2020).

213. Tang, J. et al. Exosomal miR-9-3p suppresses HBGF-5 expression and is a functional biomarker in hepatocellular carcinoma. Minerva Med. 109, 15-23 (2018).

214. Liu, W. et al. Serum exosomal miR-125b is a novel prognostic marker for hepatocellular carcinoma. OncoTargets Ther. 10, 3843-3851 (2017).

215. Shi, M. et al. Decreased levels of serum exosomal miR-638 predict poor prognosis in hepatocellular carcinoma. J. Cell. Biochem. 119, 4711-4716 (2018).

216. Sugimachi, K. et al. Identification of a bona fide microRNA biomarker in serum exosomes that predicts hepatocellular carcinoma recurrence after liver transplantation. Br. J. Cancer 112, 532-538 (2015).

217. Sohn, W. et al. Serum exosomal microRNAs as novel biomarkers for hepatocellular carcinoma. Exp. Mol. Med. 47, e184 (2015).

218. Wang, G. et al. Exosomal MiR-744 inhibits proliferation and sorafenib chemoresistance in hepatocellular carcinoma by targeting PAX2. Med. Sci. Monit. 25, 7209-7217 (2019).

219. Cao, Q. et al. Exosomal long noncoding RNAs in aging and age-related diseases. IUBMB life 71, 1846-1856 (2019).

220. Li, B. et al. LncRNA FAL1 promotes cell proliferation and migration by acting as a CeRNA of miR-1236 in hepatocellular carcinoma cells. Life Sci. 197, 122-129 (2018).

221. Wang, D. et al. Exosomal IncRNA H19 promotes the progression of hepatocellular carcinoma treated with Propofol via miR-520a-3p/LIMK1 axis. Cancer Med. 9, 7218-7230 (2020)
222. Conigliaro, A. et al. CD90+ liver cancer cells modulate endothelial cell phenotype through the release of exosomes containing H19 IncRNA. Mol. Cancer 14, 155 (2015).

223. Li, X., Lei, Y., Wu, M. \& Li, N. Regulation of macrophage activation and polarization by HCC-derived exosomal IncRNA TUC339. Int. J. Mol. Sci. 19, 2958 (2018).

224. Takahashi, K. et al. Involvement of extracellular vesicle long noncoding RNA (linc-VLDLR) in tumor cell responses to chemotherapy. Mol. cancer Res.: MCR 12, 1377-1387 (2014).

225. Takahashi, K. et al. Extracellular vesicle-mediated transfer of long non-coding RNA ROR modulates chemosensitivity in human hepatocellular cancer. FEBS Open Bio. 4, 458-467 (2014).

226. Ma, D. et al. Exosome-transferred long non-coding RNA ASMTL-AS1 contributes to malignant phenotypes in residual hepatocellular carcinoma after insufficient radiofrequency ablation. Cell Prolif. 53, e12795 (2020).

227. Sun, L. et al. Serum and exosome long non coding RNAs as potential biomarkers for hepatocellular carcinoma. J. Cancer 9, 2631-2639 (2018).

228. Xu, H., Chen, Y., Dong, X. \& Wang, X. Serum exosomal long noncoding RNAs ENSG00000258332.1 and LINC00635 for the diagnosis and prognosis of hepatocellular carcinoma. Cancer Epidemiol., Biomark. Prev. 27, 710-716 (2018).

229. Yao, Z. et al. Serum exosomal long noncoding RNAs Inc-FAM72D-3 and IncEPC1-4 as diagnostic biomarkers for hepatocellular carcinoma. Aging 12, 11843-11863 (2020).

230. Zhang, $C$. et al. IncRNA-HEIH in serum and exosomes as a potential biomarker in the HCV-related hepatocellular carcinoma. Cancer Biomark. 21, 651-659 (2018).

231. Wang, J. et al. Exosome-transmitted long non-coding RNA SENP3-EIF4A1 suppresses the progression of hepatocellular carcinoma. Aging 12, 11550-11567 (2020).

232. Zhuo, C. et al. Exosomal linc-FAM138B from cancer cells alleviates hepatocellular carcinoma progression via regulating miR-765. Aging (Albany NY) 12, 26236-26247 (2020).

233. Huang, X. Y. et al. Exosomal circRNA-100338 promotes hepatocellular carcinoma metastasis via enhancing invasiveness and angiogenesis. J. Exp. Clin. Cancer Res. 39, 20 (2020).

234. Su, Y. et al. CircRNA Cdr1as functions as a competitive endogenous RNA to promote hepatocellular carcinoma progression. Aging 11, 8183-8203 (2019).

235. Li, Y., Zang, H., Zhang, X. \& Huang, G. Exosomal Circ-ZNF652 promotes cell proliferation, migration, invasion and glycolysis in hepatocellular carcinoma via miR-29a-3p/GUCD1 axis. Cancer Manag. Res. 12, 7739-7751 (2020).

236. Lai, Z. et al. Exosomal circFBLIM1 promotes hepatocellular carcinoma progres sion and glycolysis by regulating the miR-338/LRP6 axis. Cancer Biother. Radiopharm. https://doi.org/10.1089/cbr.2020.3564 (2020).

237. Zhu, C. et al. Circular RNA hsa_circ_0004277 stimulates malignant phenotype of hepatocellular carcinoma and epithelial-mesenchymal transition of peripheral cells. Front. Cell Dev. Biol. 8, 585565 (2020).

238. Wang, G. et al. Three isoforms of exosomal circPTGR1 promote hepatocellular carcinoma metastasis via the miR449a-MET pathway. EBioMedicine 40, 432-445 (2019).

239. Liu, D. et al. Exosome-transmitted circ MMP2 promotes hepatocellular carcinoma metastasis by upregulating MMP2. Mol. Oncol. 14, 1365-1380 (2020).

240. Zhang, P. F. et al. Cancer cell-derived exosomal circUHRF1 induces natural killer cell exhaustion and may cause resistance to anti-PD1 therapy in hepatocellular carcinoma. Mol. Cancer 19, 110 (2020).

241. Luo, Y., Liu, F. \& Gui, R. High expression of circulating exosomal circAKT3 is associated with higher recurrence in HCC patients undergoing surgical treatment. Surgical Oncol. 33, 276-281 (2020).

242. Sun, X. H. et al. Serum-derived three-circRNA signature as a diagnostic biomarker for hepatocellular carcinoma. Cancer Cell Int. 20, 226 (2020).

243. Yu, Y. et al. Circular RNA hsa_circ_0061395 accelerates hepatocellular carcinoma progression via regulation of the miR-877-5p/PIK3R3 axis. Cancer cell Int. 21, 10 (2021).

244. Chen, W. et al. Exosome-transmitted circular RNA hsa_circ_0051443 suppresses hepatocellular carcinoma progression. Cancer Lett. 475, 119-128 (2020).

245. Wang, Y. et al. Downregulation of hsa_circ_0074854 suppresses the migration and invasion in hepatocellular carcinoma via interacting with HuR and via suppressing exosomes-mediated macrophage M2 polarization. Int. J. Nanomed. 16, 2803-2818 (2021).

246. Brenner, H., Kloor, M. \& Pox, C. P. Colorectal cancer. Lancet (Lond., Engl.) 383 1490-1502 (2014)

247. Zhang, N., Hu, X., Du, Y. \& Du, J. The role of miRNAs in colorectal cancer progression and chemoradiotherapy. Biomed. Pharmacother. 134, 111099 (2021).

248. Shang, A. et al. Exosomal miR-183-5p promotes angiogenesis in colorectal cancer by regulation of FOXO1. Aging 12, 8352-8371 (2020).

249. Zeng, Z. et al. Cancer-derived exosomal miR-25-3p promotes pre-metastatic niche formation by inducing vascular permeability and angiogenesis. Nat. Commun. 9, 5395 (2018). 
250. Cheng, W. C. et al. RAB27B-activated secretion of stem-like tumor exosomes delivers the biomarker microRNA-146a-5p, which promotes tumorigenesis and associates with an immunosuppressive tumor microenvironment in colorectal cancer. Int. J. Cancer 145, 2209-2224 (2019).

251. Zhang, Y. et al. Cancer-associated fibroblasts-derived exosomal miR-17-5p promotes colorectal cancer aggressive phenotype by initiating a RUNX3/MYC/ TGF- $\beta 1$ positive feedback loop. Cancer Lett. 491, 22-35 (2020).

252. Bigagli, E., Luceri, C., Guasti, D. \& Cinci, L. Exosomes secreted from human colon cancer cells influence the adhesion of neighboring metastatic cells: Role of microRNA-210. Cancer Biol. Ther. 17, 1062-1069 (2016).

253. Zhao, S. et al. Tumor-derived exosomal miR-934 induces macrophage M2 polarization to promote liver metastasis of colorectal cancer. J. Hematol. Oncol. 13, 156 (2020).

254. Zhang, X. et al. Exosomal miR-1255b-5p targets human telomerase reverse transcriptase in colorectal cancer cells to suppress epithelial-to-mesenchymal transition. Mol. Oncol. 14, 2589-2608 (2020).

255. Liu, H. et al. Colorectal cancer-derived exosomal miR-106b-3p promotes metastasis by down-regulating DLC-1 expression. Clin. Sci. (Lond., Engl.: 1979). 134, 419-434 (2020)

256. Ogata-Kawata, $\mathrm{H}$. et al. Circulating exosomal microRNAs as biomarkers of colon cancer. PLoS ONE 9, e92921 (2014).

257. Karimi, N. et al. Serum overexpression of miR-301a and miR-23a in patients with colorectal cancer. J. Chin. Med. Assoc.: JCMA 82, 215-220 (2019).

258. Fu, F., Jiang, W., Zhou, L. \& Chen, Z. Circulating exosomal miR-17-5p and miR$92 a-3 p$ predict pathologic stage and grade of colorectal cancer. Transl. Oncol. 11, 221-232 (2018)

259. Yan, S. et al. Exosomal miR-6803-5p as potential diagnostic and prognostic marker in colorectal cancer. J. Cell. Biochem. 119, 4113-4119 (2018).

260. Tang, Y. et al. Tumor-derived exosomal miRNA-320d as a biomarker for metastatic colorectal cancer. J. Clin. Lab. Anal. 33, e23004 (2019).

261. Liu, X. et al. Circulating exosomal miR-27a and miR-130a act as novel diagnostic and prognostic biomarkers of colorectal cancer. Cancer Epidemiol. Biomark. Prev. 27, 746-754 (2018)

262. Zhang, N. et al. Reduced serum exosomal miR-874 expression predicts poor prognosis in colorectal cancer. Eur. Rev. Med. Pharmacol. Sci. 24, 664-672 (2020).

263. Sun, Y. et al. Identification of serum miR-30a-5p as a diagnostic and prognostic biomarker in colorectal cancer. Cancer Biomark. 24, 299-305 (2019).

264. Liu, T. et al. Exosome-transmitted miR-128-3p increase chemosensitivity of oxaliplatin-resistant colorectal cancer. Mol. Cancer 18, 43 (2019).

265. Liang, Z. X. et al. Correction: LncRNA RPPH1 promotes colorectal cancer metastasis by interacting with TUBB3 and by promoting exosomes-mediated macrophage M2 polarization. Cell Death Dis. 11, 465 (2020).

266. Xu, J. et al. Exosomal MALAT1 sponges miR-26a/26b to promote the invasion and metastasis of colorectal cancer via FUT4 enhanced fucosylation and PI3K/ Akt pathway. J. Exp. Clin. Cancer Res. 39, 54 (2020).

267. Zhao, Y. et al. Long noncoding RNA LINC02418 regulates MELK expression by acting as a ceRNA and may serve as a diagnostic marker for colorectal cancer. Cell Death Dis. 10, 568 (2019).

268. Ren, J. et al. Carcinoma-associated fibroblasts promote the stemness and chemoresistance of colorectal cancer by transferring exosomal IncRNA H19. Theranostics 8, 3932-3948 (2018).

269. Chen, X. et al. Exosomal Long Non-coding RNA HOTTIP increases resistance of colorectal cancer cells to mitomycin via impairing MiR-214-mediated degradation of KPNA3. Front. Cell Dev. Biol. 8, 582723 (2020).

270. Oehme, F. et al. Low level of exosomal long non-coding RNA HOTTIP is a prognostic biomarker in colorectal cancer. RNA Biol. 16, 1339-1345 (2019).

271. Liu, L. et al. Prognostic and predictive value of long non-coding RNA GAS5 and mircoRNA-221 in colorectal cancer and their effects on colorectal cancer cell proliferation, migration and invasion. Cancer Biomark. 22, 283-299 (2018).

272. Liu, T. et al. Exosomal long noncoding RNA CRNDE-h as a novel serum-based biomarker for diagnosis and prognosis of colorectal cancer. Oncotarget 7, 85551-85563 (2016)

273. $\mathrm{Yu}, \mathrm{B}$. et al. Diagnostic potential of serum exosomal colorectal neoplasia differentially expressed long non-coding RNA (CRNDE-p) and microRNA-217 expression in colorectal carcinoma. Oncotarget 8, 83745-83753 (2017).

274. Wang, L. et al. Circulating long non-coding RNA colon cancer-associated transcript 2 protected by exosome as a potential biomarker for colorectal cancer. Biomed. Pharmacother. 113, 108758 (2019).

275. Hu, D. et al. Plasma exosomal long non-coding RNAs serve as biomarkers for early detection of colorectal cancer. Cell. Physiol. Biochem.51, 2704-2715 (2018).

276. $\mathrm{Yu}, \mathrm{M}$. et al. Circulating serum exosomal long non-coding RNAs FOXD2-AS1, NRIR, and XLOC_009459 as diagnostic biomarkers for colorectal cancer. Front. Oncol. 11, 618967 (2021).
277. Barbagallo, C. et al. LncRNA UCA1, upregulated in CRC biopsies and downregulated in serum exosomes, controls mRNA expression by RNA-RNA interactions. Mol. Ther. Nucleic Acids 12, 229-241 (2018).

278. Shang, A. et al. Exosomal circPACRGL promotes progression of colorectal cancer via the miR-142-3p/miR-506-3p- TGF- $\beta 1$ axis. Mol. cancer 19, 117 (2020).

279. $\mathrm{Li}$, Y. et al. A novel circFMN2 promotes tumor proliferation in $\mathrm{CRC}$ by regulating the miR-1182/hTERT signaling pathways. Clin. Sci. (Lond., Engl.: 1979). 133, 2463-2479 (2019).

280. Feng, W. et al. circlFT80 functions as a ceRNA of miR-1236-3p to promote colorectal cancer progression. Mol. Ther. Nucleic Acids 18, 375-387 (2019).

281. Wang, X. et al. Exosome-delivered circRNA promotes glycolysis to induce chemoresistance through the miR-122-PKM2 axis in colorectal cancer. Mol. Oncol. 14, 539-555 (2020).

282. Yang, H. et al. Hypoxia induced exosomal circRNA promotes metastasis of colorectal cancer via targeting GEF-H1/RhoA axis. Theranostics 10, 8211-8226 (2020).

283. Pan, B. et al. Identification of serum exosomal hsa-circ-0004771 as a novel diagnostic biomarker of colorectal cancer. Front. Genet. 10, 1096 (2019).

284. Smyth, E. C. et al. Gastric cancer. Lancet (Lond., Engl.) 396, 635-648 (2020).

285. Huang, J. et al. Exosome-mediated transfer of miR-1290 promotes cell proliferation and invasion in gastric cancer via NKD1. Acta Biochim. Biophys. Sin. 51, 900-907 (2019).

286. Wang, M. et al. Deregulated microRNAs in gastric cancer tissue-derived mesenchymal stem cells: novel biomarkers and a mechanism for gastric cancer. Br. J. cancer 110, 1199-1210 (2014).

287. Xia, X. et al. Hypoxic gastric cancer-derived exosomes promote progression and metastasis via MiR-301a-3p/PHD3/HIF-1a positive feedback loop. Oncogene 39, 6231-6244 (2020).

288. Wei, S. et al. Exosomal transfer of miR-15b-3p enhances tumorigenesis and malignant transformation through the DYNLT1/Caspase-3/Caspase-9 signaling pathway in gastric cancer. J. Exp. Clin. Cancer Res. 39, 32 (2020).

289. Shi, L. et al. Exosomal miRNA-34 from cancer-associated fibroblasts inhibits growth and invasion of gastric cancer cells in vitro and in vivo. Aging 12, 8549-8564 (2020).

290. Ohshima, K. et al. Let-7 microRNA family is selectively secreted into the extracellular environment via exosomes in a metastatic gastric cancer cell line. PLoS ONE 5, e13247 (2010).

291. Li, Q. et al. Exosomal miR-21-5p derived from gastric cancer promotes peritoneal metastasis via mesothelial-to-mesenchymal transition. Cell death Dis. 9, 854 (2018).

292. Wang, J. et al. Exosomal miR-27a derived from gastric cancer cells regulates the transformation of fibroblasts into cancer-associated fibroblasts. Cell. Physiol. Biochem. 49, 869-883 (2018).

293. Xu, G. et al. Exosomal miRNA-139 in cancer-associated fibroblasts inhibits gastric cancer progression by repressing MMP11 expression. Int. J. Biol. Sci. 15, 2320-2329 (2019).

294. Yang, H. et al. Exosome-derived miR-130a activates angiogenesis in gastric cancer by targeting C-MYB in vascular endothelial cells. Mol. Ther. 26, 2466-2475 (2018).

295. Wang, N. et al. A serum exosomal microRNA panel as a potential biomarker test for gastric cancer. Biochem. Biophys. Res. Commun. 493, 1322-1328 (2017).

296. Shi, Y. et al. Exosomal miR-1246 in serum as a potential biomarker for early diagnosis of gastric cancer. Int. J. Clin. Oncol. 25, 89-99 (2020).

297. Ren, W. et al. Exosomal miRNA-107 induces myeloid-derived suppressor cell expansion in gastric cancer. Cancer Manag. Res. 11, 4023-4040 (2019).

298. Feng, C. et al. Exosomal miR-196a-1 promotes gastric cancer cell invasion and metastasis by targeting SFRP1. Nanomed. (Lond., Engl.) 14, 2579-2593 (2019).

299. Zhu, M., Zhang, N., He, S. \& Lu, X. Exosomal miR-106a derived from gastric cancer promotes peritoneal metastasis via direct regulation of Smad7. Cell Cycle (Georget., Tex.) 19, 1200-1221 (2020).

300. Shi, S. S. et al. Exosomal miR-155-5p promotes proliferation and migration of gastric cancer cells by inhibiting TP53INP1 expression. Pathol. Res. Pract. 216, 152986 (2020)

301. Zhang, J. et al. HOTAIR contributes to the carcinogenesis of gastric cancer via modulating cellular and exosomal miRNAs level. Cell death Dis. 11, 780 (2020).

302. Wang, L. et al. Exosome-transferred LINC01559 promotes the progression of gastric cancer via PI3K/AKT signaling pathway. Cell Death Dis. 11, 723 (2020).

303. Lu, Y. et al. Exosome-delivered LncHEIH promotes gastric cancer progression by upregulating $\mathrm{EZH} 2$ and stimulating methylation of the GSDME promoter. Front. Cell Dev. Biol. 8, 571297 (2020).

304. Wang, J. et al. Exosome-mediated transfer of IncRNA HOTTIP promotes cisplatin resistance in gastric cancer cells by regulating $\mathrm{HMGA} 1 / \mathrm{miR}-218$ axis. OncoTargets Ther. 12, 11325-11338 (2019).

305. Lin, L. Y. et al. Tumor-originated exosomal IncUEGC1 as a circulating biomarker for early-stage gastric cancer. Mol. cancer 17, 84 (2018). 
306. Zhao, R. et al. Exosomal long noncoding RNA HOTTIP as potential novel diagnostic and prognostic biomarker test for gastric cancer. Mol. Cancer 17, 68 (2018).

307. Guo, X. et al. Circulating exosomal gastric cancer-associated long noncoding RNA1 as a biomarker for early detection and monitoring progression of gastric cancer: a multiphase study. JAMA Surg. 155, 572-579 (2020).

308. $\mathrm{Xu}, \mathrm{H}$. et al. Identification of serum exosomal IncRNA MIAT as a novel diagnostic and prognostic biomarker for gastric cancer. J. Clin. Lab. Anal. 34, e23323 (2020).

309. Zhou, H. et al. Circulating exosomal long non-coding RNA H19 as a potential novel diagnostic and prognostic biomarker for gastric cancer. J. Int. Med. Res. 48, 300060520934297 (2020).

310. Zheng, P. et al. Plasma exosomal long noncoding RNA Inc-SLC2A12-10:1 as a novel diagnostic biomarker for gastric cancer. OncoTargets Ther. 13, 4009-4018 (2020).

311. Piao, H. Y., Guo, S., Wang, Y. \& Zhang, J. Exosomal long non-coding RNA CEBPAAS1 inhibits tumor apoptosis and functions as a non-invasive biomarker for diagnosis of gastric cancer. OncoTargets Ther. 13, 1365-1374 (2020).

312. $\mathrm{Li}$, S. et al. Exosomal long noncoding RNA Inc-GNAQ-6:1 may serve as a diagnostic marker for gastric cancer. Clin. Chim. Acta. 501, 252-257 (2020).

313. Cai, C. et al. Serum exosomal long noncoding RNA pcsk2-2:1 as a potential novel diagnostic biomarker for gastric cancer. OncoTargets Ther. 12, 10035-10041 (2019).

314. Xie, M. et al. Exosomal circSHKBP1 promotes gastric cancer progression via regulating the miR-582-3p/HUR/VEGF axis and suppressing HSP90 degradation. Mol. Cancer 19, 112 (2020).

315. Zhang, H. et al. Exosomal circRNA derived from gastric tumor promotes white adipose browning by targeting the miR-133/PRDM16 pathway. Int. J. Cancer 144, 2501-2515 (2019).

316. $\mathrm{Yu}$, L. et al. Plasma exosomal CircNEK9 accelerates the progression of gastric cancer via miR-409-3p/MAP7 Axis. Dig, Dis. Sci. https://doi.org/10.1007/s10620020-06816-z (2021).

317. Li, S. et al. Gastric cancer derived exosomes mediate the delivery of circRNA to promote angiogenesis by targeting miR-29a/VEGF axis in endothelial cells. Biochem. Biophys. Res. Commun. 560, 37-44 (2021).

318. Hui, C., Tian, L. \& He, X. Circular RNA circNHSL1 contributes to gastric cancer progression through the miR-149-5p/YWHAZ Axis. Cancer Manag. Res. 12, 7117-7130 (2020).

319. Zhong, Y. et al. Circular RNA circ_0032821 contributes to oxaliplatin (OXA) resistance of gastric cancer cells by regulating SOX9 via miR-515-5p. Biotechnol. Lett. 43, 339-351 (2021)

320. Shao, Y. et al. Hsa_circ_0065149 is an Indicator for Early Gastric Cancer Screening and Prognosis Prediction. Pathol. Oncol. Res 26, 1475-1482 (2020).

321. Ren, S. et al. RNA-seq analysis of prostate cancer in the Chinese population identifies recurrent gene fusions, cancer-associated long noncoding RNAs and aberrant alternative splicings. Cell Res. 22, 806-821 (2012).

322. Litwin, M. S. \& Tan, H. J. The Diagnosis and Treatment of Prostate Cancer: A Review. JAMA 317, 2532-2542 (2017).

323. Wang, J. et al. Exosomal microRNAs as liquid biopsy biomarkers in prostate cancer. Crit. Rev. Oncol. Hematol. 145, 102860 (2020).

324. Zhou, C. et al. Functional implication of exosomal miR-217 and miR-23b-3p in the progression of prostate cancer. OncoTargets Ther. 13, 11595-11606 (2020).

325. Jiang, S. et al. Human bone marrow mesenchymal stem cells-derived microRNA205-containing exosomes impede the progression of prostate cancer through suppression of RHPN2. J. Exp. Clin. cancer Res. 38, 495 (2019).

326. Wang, $X$. et al. Prostate carcinoma cell-derived exosomal MicroRNA-26a modulates the metastasis and tumor growth of prostate carcinoma. Biomed, Pharmacother. 117, 109109 (2019).

327. Guan, $\mathrm{H}$. et al. Tumor-associated macrophages promote prostate cancer progression via exosome-mediated miR-95 transfer. J. Cell. Physiol. 235, 9729-9742 (2020).

328. Dai, Y. \& Gao, X. Inhibition of cancer cell-derived exosomal microRNA-183 suppresses cell growth and metastasis in prostate cancer by upregulating TPM1. Cancer Cell Int. 21, 145 (2021).

329. Ye, Y. et al. Exosomal miR-141-3p regulates osteoblast activity to promote the osteoblastic metastasis of prostate cancer. Oncotarget 8, 94834-94849 (2017).

330. Huang, X. et al. Exosomal miR-1290 and miR-375 as prognostic markers in castration-resistant prostate cancer. Eur. Urol. 67, 33-41 (2015).

331. Li, Z. et al. Exosomal microRNA-141 is upregulated in the serum of prostate cancer patients. OncoTargets Ther. 9, 139-148 (2016).

332. Hessvik, N. P., Sandvig, K. \& Llorente, A. Exosomal miRNAs as biomarkers for prostate cancer. Front. Genet. 4, 36 (2013).

333. Wang, J. et al. Long non-coding RNA MYU promotes prostate cancer proliferation by mediating the miR-184/c-Myc axis. Oncol. Rep. 40, 2814-2825 (2018).

334. Wang, Y. H. et al. Tumor-derived exosomal long noncoding RNAs as promising diagnostic biomarkers for prostate cancer. Cell. Physiol. Biochem. 46, 532-545 (2018).
335. Ozgur, E. \& Gezer, U. Investigation of IncRNA H19 in prostate cancer cells and secreted exosomes upon androgen stimulation or androgen receptor blockage. Bratisl. Lek. Listy 121, 362-365 (2020).

336. Fang, J., Qi, J., Dong, X. \& Luo, J. Perspectives on circular RNAs as prostate cancer biomarkers. Front. Cell Dev. Biol. 8, 594992 (2020).

337. Li, T., Sun, X. \& Chen, L. Exosome circ 0044516 promotes prostate cancer cell proliferation and metastasis as a potential biomarker. J. Cell. Biochem. 121, 2118-2126 (2020).

338. Zhang, $\mathrm{H}$. et al. Exosomal Circ-XIAP promotes docetaxel resistance in prostate cancer by regulating miR-1182/TPD52 axis. Drug Des. Dev. Ther. 15, 1835-1849 (2021).

339. Ariston Gabriel, A. N. et al. The involvement of exosomes in the diagnosis and treatment of pancreatic cancer. Mol. Cancer 19, 132 (2020).

340. Vijan, S. Type 2 diabetes. Ann. Intern. Med. 171, ITC65-ITC80 (2019).

341. Zheng, Y., Ley, S. H. \& Hu, F. B. Global aetiology and epidemiology of type 2 diabetes mellitus and its complications. Nat. Rev. Endocrinol. 14, 88-98 (2018).

342. Li, C.-J., Fang, Q.-H., Liu, M.-L. \& Lin, J.-N. Current understanding of the role of adipose-derived extracellular vesicles in metabolic homeostasis and diseases: communication from the distance between cells/tissues. Theranostics 10, 7422-7435 (2020).

343. Galic, S., Oakhill, J. S. \& Steinberg, G. R. Adipose tissue as an endocrine organ Mol. Cell. Endocrinol. 316, 129-139 (2010).

344. Thomou, T. et al. Adipose-derived circulating miRNAs regulate gene expression in other tissues. Nature 542, 450-455 (2017).

345. Gesmundo, I. et al. Adipocyte-derived extracellular vesicles regulate survival and function of pancreatic $\beta$-cells.JCl insight 6, e141962 (2021).

346. Pan, Y. et al. Adipocyte-secreted exosomal microRNA-34a inhibits M2 macrophage polarization to promote obesity-induced adipose inflammation. J. Clin. Investig. 129, 834-849 (2019).

347. Li, D. et al.Gonadal white adipose tissue-derived exosomal MiR-222 promotes obesity-associated insulin resistance. Aging 12, 22719-22743 (2020).

348. Yu, Y. et al. Adipocyte-derived exosomal MiR-27a induces insulin resistance in skeletal muscle through repression of PPARy. Theranostics 8, 2171-2188 (2018).

349. Dang, S.-Y. et al. Exosomal transfer of obesity adipose tissue for decreased miR141-3p mediate insulin resistance of hepatocytes. Int. J. Biol. Sci. 15, 351-368 (2019).

350. Gan, L. et al. Small extracellular microvesicles mediated pathological communications between dysfunctional adipocytes and cardiomyocytes as a novel mechanism exacerbating ischemia/reperfusion injury in diabetic mice. Circulation 141, 968-983 (2020).

351. Ying, W. et al. Adipose tissue macrophage-derived exosomal miRNAs can modulate in vivo and in vitro insulin sensitivity. Cell 171, 372-384.e312 (2017).

352. Liu, T., Sun, Y.-C., Cheng, P. \& Shao, H.-G. Adipose tissue macrophage-derived exosomal miR-29a regulates obesity-associated insulin resistance. Biochem. Biophys. Res. Commun. 515, 352-358 (2019).

353. Ying, W. et al. MiR-690, an exosomal-derived miRNA from M2-polarized mac rophages, improves insulin sensitivity in obese mice. Cell Metab 33, 781-790.e5 (2021).

354. Tian, F. et al. miR-210 in Exosomes Derived from Macrophages under High Glucose Promotes Mouse Diabetic Obesity Pathogenesis by Suppressing NDUFA4 Expression. J. Diabetes Res. 2020, 6894684 (2020).

355. Wu, J. et al. Hepatic exosome-derived miR-130a-3p attenuates glucose intolerance via suppressing PHLPP2 gene in adipocyte. Metab.: Clin. Exp. 103, 154006 (2020).

356. Fu, Q. et al. Hepatocytes derived extracellular vesicles from high-fat diet induced obese mice modulate genes expression and proliferation of islet $\beta$ cells. Biochem. Biophys. Res. Commun. 516, 1159-1166 (2019).

357. Jalabert, A. et al. Exosome-like vesicles released from lipid-induced insulinresistant muscles modulate gene expression and proliferation of beta recipient cells in mice. Diabetologia 59, 1049-1058 (2016).

358. Zhang, A. et al. Islet $\beta$ cell: An endocrine cell secreting miRNAs. Biochem. Biophys. Res. Commun. 495, 1648-1654 (2018).

359. Li, J. et al. Pancreatic $\beta$ cells control glucose homeostasis via the secretion of exosomal miR-29 family. J. Extracell. Vesicles 10, e12055 (2021).

360. $\mathrm{Xu}, \mathrm{H}$. et al. Pancreatic $\beta$ cell microRNA-26a alleviates type 2 diabetes by improving peripheral insulin sensitivity and preserving $\beta$ cell function. PLOS Biol. 18, e3000603 (2020).

361. Kamalden, T. A. et al. Exosomal MicroRNA-15a transfer from the pancreas augments diabetic complications by inducing oxidative stress. Antioxid. Redox Signal 27, 913-930 (2017).

362. Shen, Z. et al. MicroRNA-127 inhibits cell proliferation via targeting Kif3b in pancreatic $\beta$ cells. Aging 11, 1342-1355 (2019).

363. Katayama, M. et al. Circulating exosomal miR-20b-5p is elevated in type 2 diabetes and could impair insulin action in human skeletal muscle. Diabetes $\mathbf{6 8}$, 515-526 (2019). 
364. Santovito, D. et al. Plasma exosome microRNA profiling unravels a new potential modulator of adiponectin pathway in diabetes: effect of glycemic control. J. Clin. Endocrinol. Metab. 99, E1681-E1685 (2014).

365. Ruan, Y. et al. Circulating LncRNAs analysis in patients with type 2 diabetes reveals novel genes influencing glucose metabolism and Islet $\beta$-cell function. Cell. Physiol. Biochem. 46, 335-350 (2018).

366. Tello-Flores, V. A. et al. Altered levels of MALAT1 and H19 derived from serum or serum exosomes associated with type-2 diabetes. Non-coding RNA Res. 5, 71-76 (2020).

367. Li, B. et al. The MSC-derived exosomal IncRNA H19 promotes wound healing in diabetic foot ulcers by upregulating PTEN via MicroRNA-152-3p. Mol. Ther. Nucleic Acids 19, 814-826 (2020).

368. Tao, S.-C. et al. Extracellular vesicle-mimetic nanovesicles transport LncRNA-H19 as competing endogenous RNA for the treatment of diabetic wounds. Drug Deliv. 25, 241-255 (2018).

369. Cao, X. et al. MSC-derived exosomal IncRNA SNHG7 suppresses endothelialmesenchymal transition and tube formation in diabetic retinopathy via miR34a-5p/XBP1 axis. Life Sci. 272, 119232 (2021).

370. Wang, S. et al. CircRNA-0077930 from hyperglycaemia-stimulated vascular endothelial cell exosomes regulates senescence in vascular smooth muscle cells. Cell Biochem Funct. 38, 1056-1068 (2020).

371. Chen, Z.-J. et al. Serum and exosomal hsa_circ_0000907 and hsa_circ_0057362 as novel biomarkers in the early diagnosis of diabetic foot ulcer. Eur. Rev. Med. Pharmacol. Sci. 24, 8117-8126 (2020).

372. Liu, C. et al. Targeting pericyte-endothelial cell crosstalk by circular RNAcPWWP2A inhibition aggravates diabetes-induced microvascular dysfunction. Proc. Natl Acad. Sci. USA 116, 7455-7464 (2019).

373. Bai, S. et al. Exosomal circ_DLGAP4 promotes diabetic kidney disease progression by sponging miR-143 and targeting ERBB3/NF-KB/MMP-2 axis. Cell Death Dis. 11, 1008 (2020).

374. WHO. Obesity. Available online at: https://www.who.int/health-topics/ obesity\#tab=tab_1 (2021).

375. Afshin, A. et al. Health Effects of Overweight and Obesity in 195 Countries over 25 Years. N. Engl. J. Med. 377, 13-27 (2017)

376. Larabee, C. M., Neely, O. C. \& Domingos, A. I. Obesity: a neuroimmunometabolic perspective. Nat. Rev. Endocrinol. 16, 30-43 (2020).

377. Santamaria-Martos, F. et al. Comparative and functional analysis of plasma membrane-derived extracellular vesicles from obese vs. nonobese women. Clin. Nutr. (Edinb., Scotl.) 39, 1067-1076 (2020).

378. Wang, J. et al. Elafin inhibits obesity, hyperglycemia, and liver steatosis in highfat diet-treated male mice. Sci. Rep. 10, 12785 (2020).

379. Mendonça, M. D. et al. Aerobic exercise training regulates serum extracellular vesicle miRNAs linked to obesity to promote their beneficial effects in mice. Am. J. Physiol. Endocrinol. Metab. 319, E579-E591 (2020).

380. Hubal, M. J. et al. Circulating adipocyte-derived exosomal MicroRNAs associated with decreased insulin resistance after gastric bypass. Obesity (Silver Spring, Md.) 25, 102-110 (2017)

381. Bae, Y.-U. et al. Bariatric surgery alters microRNA content of circulating exosomes in patients with obesity. Obesity (Silver Spring, Md.) 27, 264-271 (2019).

382. Ferrante, S. C. et al. Adipocyte-derived exosomal miRNAs: a novel mechanism for obesity-related disease. Pediatr. Res. 77, 447-454 (2014).

383. Yang, Z., Wei, Z., Wu, X. \& Yang, H. Screening of exosomal miRNAs derived from subcutaneous and visceral adipose tissues: Determination of targets for the treatment of obesity and associated metabolic disorders. Mol. Med. Rep. 18, 3314-3324 (2018).

384. Kim, Y. \& Kim, O.-K. Potential roles of adipocyte extracellular vesicle-derived miRNAs in obesity-mediated insulin resistance. Adv. Nutr 12,566-574 (2020).

385. Kim, H. et al. Effect of diabetes on exosomal miRNA profile in patients with obesity. BMJ Open Diabetes Res. Care 8, e001403 (2020).

386. Compston, J. E., McClung, M. R. \& Leslie, W. D. Osteoporosis. Lancet 393, 364-376 (2019).

387. Hendrickx, G., Boudin, E. \& van Hul, W. A look behind the scenes: the risk and pathogenesis of primary osteoporosis. Nat. Rev. Rheumatol. 11, 462-474 (2015).

388. Li, G. et al. An overview of osteoporosis and frailty in the elderly. BMC Musculoskelet. Disord. 18, 46 (2017).

389. Fuggle, N. R. et al. Fracture prediction, imaging and screening in osteoporosis. Nat. Rev. Endocrinol. 15, 535-547 (2019).

390. $\mathrm{Xu}, \mathrm{T}$. et al. Exosomal miRNA-128-3p from mesenchymal stem cells of aged rats regulates osteogenesis and bone fracture healing by targeting Smad5. J. Nanobiotechnol. 18, 47 (2020).

391. Takafuji, Y. et al. MicroRNA-196a-5p in extracellular vesicles secreted from myoblasts suppresses osteoclast-like cell formation in mouse cells.Tissue Int 108, 364-376 (2021).
392. Wang, X. et al. Mesenchymal stem cell-derived exosomes have altered microRNA profiles and induce osteogenic differentiation depending on the stage of differentiation. PLOS ONE 13, e0193059 (2018).

393. $\mathrm{Xu}, \mathrm{J}$.-F. et al. Altered MicroRNA expression profile in exosomes during osteogenic differentiation of human bone marrow-derived mesenchymal stem cells. PLOS ONE. 9, e114627 (2014).

394. Jiang, L.-B., Tian, L. \& Zhang, C.-G. Bone marrow stem cells-derived exosomes extracted from osteoporosis patients inhibit osteogenesis via microRNA-21/ SMAD7. Eur. Rev. Med. Pharmacol. Sci. 22, 6221-6229 (2018).

395. Sun, W. et al. Osteoclast-derived microRNA-containing exosomes selectively inhibit osteoblast activity. Cell Discov. 2, 16015 (2016).

396. Li, D. et al. Osteoclast-derived exosomal miR-214-3p inhibits osteoblastic bone formation. Nat. Commun. 7, 10872 (2016).

397. Yang, J.-X. et al. Osteoclast-derived miR-23a-5p-containing exosomes inhibit osteogenic differentiation by regulating Runx2. Cell. Signal. 70, 109504 (2020).

398. Fulzele, S. et al. Muscle-derived miR-34a increases with age in circulating extracellular vesicles and induces senescence of bone marrow stem cells. Aging 11, 1791-1803 (2019).

399. Zainabadi, K., Liu, C. J. \& Guarente, L. SIRT1 is a positive regulator of the master osteoblast transcription factor, RUNX2. PLoS ONE. 12, e0178520 (2017).

400. Chen, Y. et al. SIRT1, a promising regulator of bone homeostasis. Life Sci. 269, 119041 (2021).

401. Qin, Y. et al. Myostatin inhibits osteoblastic differentiation by suppressing osteocyte-derived exosomal microRNA-218: A novel mechanism in muscle-bone communication. J. Biol. Chem. 292, 11021-11033 (2017).

402. Teng, Z. et al. Osteoporosis is characterized by altered expression of exosomal long non-coding RNAs. Front. Genet. 11, 566959 (2020).

403. Cui, Y. et al. EPC-derived exosomes promote osteoclastogenesis through LncRNA-MALAT1. J. Cell. Mol. Med. 23, 3843-3854 (2019).

404. Zhi, F. et al. Exosomal hsa_circ_0006859 is a potential biomarker for postmenopausal osteoporosis and enhances adipogenic versus osteogenic differentiation in human bone marrow mesenchymal stem cells by sponging miR431-5p. Stem Cell Res. Ther. 12, 157 (2021)

405. Liu, Z. et al. CircHmbox 1 targeting miRNA-1247-5p is involved in the regulation of bone metabolism by TNF-a in postmenopausal osteoporosis. Front. Cell Dev. Biol 8, 594785 (2020).

406. Zheng, D. H. et al. Erythropoietin attenuates high glucose-induced oxidative stress and inhibition of osteogenic differentiation in periodontal ligament stem cell (PDLSCs). Chem. Biol. Interact. 305, 40-47 (2019).

407. Libby, P., Ridker, P. M. \& Hansson, G. K. Leducq Transatlantic Network on Atherothrombosis Inflammation in atherosclerosis: from pathophysiology to practice. J. Am. Coll. Cardiol. 54, 2129-2138 (2009).

408. Libby, P., Ridker, P. M. \& Hansson, G. K. Progress and challenges in translating the biology of atherosclerosis. Nature 473, 317-325 (2011).

409. Barile, L. et al. Extracellular vesicles from human cardiac progenitor cells inhibit cardiomyocyte apoptosis and improve cardiac function after myocardial infarction. Cardiovasc Res. 103, 530-541 (2014).

410. Pfeifer, P., Werner, N. \& Jansen, F. Role and function of microRNAs in extracellular vesicles in cardiovascular biology. Biomed. Res Int. 2015, 161393 (2015).

411. Rezaie, J. et al. Cardioprotective role of extracellular vesicles: a highlight on exosome beneficial effects in cardiovascular diseases. J. Cell Physiol. 234, 21732-21745 (2019).

412. Qiao, S. et al. Extracellular vesicles derived from Kruppel-Like Factor 2-overexpressing endothelial cells attenuate myocardial ischemia-reperfusion injury by preventing Ly6C(high) monocyte recruitment. Theranostics 10, 11562-11579 (2020).

413. Davidson, S. M. et al. Circulating blood cells and extracellular vesicles in acute cardioprotection. Cardiovasc Res. 115, 1156-1166 (2019).

414. Kopin, L. \& Lowenstein, C. Dyslipidemia. Ann. Intern. Med. 167, ITC81-ITC96 (2017).

415. Fuchs, F. D. \& Whelton, P. K. High blood pressure and cardiovascular disease. Hypertension 75, 285-292 (2020).

416. Bress, A. P. et al. Potential cardiovascular disease events prevented with adoption of the 2017 American College of Cardiology/American Heart Association Blood Pressure Guideline. Circulation 139, 24-36 (2019).

417. Brunström, M. \& Carlberg, B. Association of blood pressure lowering with mortality and cardiovascular disease across blood pressure levels: a systematic review and meta-analysis. JAMA Intern. Med. 178, 28-36 (2018).

418. Tan, P. P. S. et al. Exosomal microRNAs in the development of essential hypertension and its potential as biomarkers. Am. J. Physiol. Heart Circ. Physiol. 320, H1486-h1497 (2021).

419. Jayaseelan, V. P. \& Arumugam, P. Exosomal microRNAs as a promising theragnostic tool for essential hypertension. Hypertens. Res 43, 74-75 (2020). 
420. Xu, F. et al. Melatonin alleviates vascular calcification and ageing through exosomal miR-204/miR-211 cluster in a paracrine manner. J. Pineal. Res 68 e12631 (2020).

421. Liu, X. et al. miRNA profiling of exosomes from spontaneous hypertensive rats using next-generation sequencing. J. Cardiovasc. Transl. Res. 12, 75-83 (2019).

422. Ren, X. S. et al. MiR155-5p in adventitial fibroblasts-derived extracellular vesicles inhibits vascular smooth muscle cell proliferation via suppressing angiotensinconverting enzyme expression. J. Extracell. Vesicles 9, 1698795 (2020).

423. Zou, X. et al. Secreted monocyte miR-27a, via mesenteric arterial mas receptoreNOS pathway, causes hypertension. Am. J. Hypertens. 33, 31-42 (2020).

424. Osada-Oka, M. et al. Macrophage-derived exosomes induce inflammatory factors in endothelial cells under hypertensive conditions. Hypertens. Res 40, 353-360 (2017).

425. Oh, J. et al. Macrophage secretion of miR-106b-5p causes renin-dependent hypertension. Nat. Commun. 11, 4798 (2020).

426. O'Malley, P. G. et al. Management of dyslipidemia for cardiovascular disease risk reduction: synopsis of the 2020 updated U.S. Department of Veterans Affairs and U.S. Department of Defense Clinical Practice Guideline. Ann. Intern Med. 173, 822-829 (2020).

427. Plakht, Y., Shiyovich, A. \& Gilutz, H. Predictors of long-term (10-year) mortality postmyocardial infarction: age-related differences. Soroka Acute Myocardial Infarction (SAMI) Project. J. Cardiol. 65, 216-223 (2015).

428. Roth, G. A. et al. Global, regional, and national burden of cardiovascular diseases for 10 causes, 1990 to 2015. J. Am. Coll. Cardiol. 70, 1-25 (2017).

429. Benjamin, E. J. et al. Heart disease and stroke statistics-2017 update: a report from the American Heart Association. Circulation 135, e146-e603 (2017)

430. Zheng, B. et al. Exosome-mediated miR-155 transfer from smooth muscle cells to endothelial cells induces endothelial injury and promotes atherosclerosis. Mol. Ther. 25, 1279-1294 (2017).

431. Zhang, Z. et al. Exosomal LINC01005 derived from oxidized low-density lipoprotein-treated endothelial cells regulates vascular smooth muscle cell phenotypic switch. Biofactors 46, 743-753 (2020).

432. Zhu, J. et al. Exosomes from nicotine-stimulated macrophages accelerate atherosclerosis through miR-21-3p/PTEN-mediated VSMC migration and proliferation. Theranostics 9, 6901-6919 (2019).

433. Iaconetti, C., Sorrentino, S., De Rosa, S. \& Indolfi, C. Exosomal miRNAs in heart disease. Physiol. (Bethesda) 31, 16-24 (2016).

434. Tan, M. et al. Thrombin stimulated platelet-derived exosomes inhibit plateletderived growth factor receptor-beta expression in vascular smooth muscle cells. Cell Physiol. Biochem. 38, 2348-2365 (2016).

435. $\mathrm{Li}$, J. et al. Thrombin-activated platelet-derived exosomes regulate endothelial cell expression of ICAM-1 via microRNA-223 during the thrombosisinflammation response. Thromb. Res. 154, 96-105 (2017).

436. Liu, J. et al. The role of exosomal non-coding RNAs in coronary artery disease. Front. Pharm. 11, 603104 (2020).

437. Loyer, X. et al. Inhibition of microRNA-92a prevents endothelial dysfunction and atherosclerosis in mice. Circ. Res. 114, 434-443 (2014).

438. Xing, X. et al. Adipose-derived mesenchymal stem cells-derived exosomemediated microRNA-342-5p protects endothelial cells against atherosclerosis. Aging (Albany NY) 12, 3880-3898 (2020).

439. Liu, Y. et al. Exosome-mediated miR-106a-3p derived from ox-LDL exposed macrophages accelerated cell proliferation and repressed cell apoptosis of human vascular smooth muscle cells. Eur. Rev. Med Pharm. Sci. 24, 7039-7050 (2020).

440. Chen, L. et al. Exosomal IncRNA GAS5 regulates the apoptosis of macrophages and vascular endothelial cells in atherosclerosis. PLOS ONE 12, e0185406 (2017).

441. Li, H. et al. Loss of exosomal MALAT1 from ox-LDL-treated vascular endothelia cells induces maturation of dendritic cells in atherosclerosis development. Cell Cycle 18, 2255-2267 (2019).

442. Huang, C. et al. Exosomal MALAT1 derived from oxidized low-density lipoprotein-treated endothelial cells promotes M2 macrophage polarization. Mol. Med. Rep. 18, 509-515 (2018)

443. Gao, H. et al. Exosomal MALAT1 derived from ox-LDL-treated endothelial cells induce neutrophil extracellular traps to aggravate atherosclerosis. Biol. Chem. 401, 367-376 (2020).

444. $\mathrm{Yu}, \mathrm{Z}$. et al. CircRNAs open a new era in the study of cardiovascular disease (Review). Int. J. Mol. Med 47, 49-64 (2021).

445. Wen, Y. et al. circRNA0006896miR1264DNMT1 axis plays an important role in carotid plaque destabilization by regulating the behavior of endothelial cells in atherosclerosis. Mol. Med. Rep 23, 311 (2021).

446. Feld, S., Kjellgren, O. \& Smalling, R. W. Aggressive interventional treatment of acute myocardial infarction. Lessons from the animal laboratory applied to the catheterization suite. Cardiology. 86, 365-373 (1995).

447. Ong, S. G. et al. Cross talk of combined gene and cell therapy in ischemic heart disease: role of exosomal microRNA transfer. Circulation 130, S60-S69 (2014).
448. Shyu, K. G. et al. Hyperbaric oxygen-induced long non-coding RNA MALAT1 exosomes suppress MicroRNA-92a expression in a rat model of acute myocardial infarction. J. Cell Mol. Med. 24, 12945-12954 (2020).

449. Huang, P. et al. Atorvastatin enhances the therapeutic efficacy of mesenchymal stem cells-derived exosomes in acute myocardial infarction via up-regulating long non-coding RNA H19. Cardiovasc Res. 116, 353-367 (2020).

450. Zhu, L. P. et al. Hypoxia-elicited mesenchymal stem cell-derived exosomes facilitates cardiac repair through miR-125b-mediated prevention of cell death in myocardial infarction. Theranostics 8, 6163-6177 (2018).

451. De Rosa, S. et al. Transcoronary concentration gradients of circulating microRNAs. Circulation 124, 1936-1944 (2011).

452. Feng, Y. et al. Ischemic preconditioning potentiates the protective effect of stem cells through secretion of exosomes by targeting Mecp2 via miR-22. PLoS ONE 9, e88685 (2014).

453. Wang, K. et al. Enhanced cardioprotection by human endometrium mesenchymal stem cells driven by exosomal microRNA-21. Stem Cells Transl. Med. 6, 209-222 (2017).

454. Li, Y. et al. Exosomal miR-301 derived from mesenchymal stem cells protects myocardial infarction by inhibiting myocardial autophagy. Biochem Biophys. Res Commun. 514, 323-328 (2019).

455. Sahoo, S. \& Losordo, D. W. Exosomes and cardiac repair after myocardial infarction. Circ. Res. 114, 333-344 (2014).

456. Khan, M. et al. Embryonic stem cell-derived exosomes promote endogenous repair mechanisms and enhance cardiac function following myocardial infarction. Circ. Res. 117, 52-64 (2015).

457. Saparov, A. et al. Role of the immune system in cardiac tissue damage and repair following myocardial infarction. Inflamm. Res 66, 739-751 (2017).

458. Peng, $Y$. et al. Exosomal miR-25-3p from mesenchymal stem cells alleviates myocardial infarction by targeting pro-apoptotic proteins and EZH2. Cell Death Dis. 11, 317 (2020).

459. Wen, Z. et al. Mesenchymal stem cell-derived exosomes ameliorate cardiomyocyte apoptosis in hypoxic conditions through microRNA144 by targeting the PTEN/AKT pathway. Stem Cell Res Ther. 11, 36 (2020).

460. Wang, W. et al. Exosomes derived miR-126 attenuates oxidative stress and apoptosis from ischemia and reperfusion injury by targeting ERRFI1. Gene 690, 75-80 (2019).

461. Pan, J. et al. Exosomes derived from miR-146a-modified adipose-derived stem cells attenuate acute myocardial infarction-induced myocardial damage via downregulation of early growth response factor 1. J. Cell Biochem. 120, 4433-4443 (2019).

462. Chen, Z. et al. Expression level and diagnostic value of exosomal NEAT1/miR204/MMP-9 in acute ST-segment elevation myocardial infarction. IUBMB Life 72 2499-2507 (2020).

463. Wang, L. \& Zhang, J. Exosomal IncRNA AK139128 derived from hypoxic cardiomyocytes promotes apoptosis and inhibits cell proliferation in cardiac fibroblasts. Int J. Nanomed. 15, 3363-3376 (2020).

464. Liew, C. C. \& Dzau, V. J. Molecular genetics and genomics of heart failure. Nat. Rev. Genet 5, 811-825 (2004).

465. Melman, Y. F., Shah, R. \& Das, S. MicroRNAs in heart failure: is the picture becoming less miRky? Circ. Heart Fail 7, 203-214 (2014).

466. Wang, L. et al. Reduced exosome miR-425 and miR-744 in the plasma represents the progression of fibrosis and heart failure. Kaohsiung J. Med Sci. 34, 626-633 (2018).

467. Wijffels, M. C., Kirchhof, C. J., Dorland, R. \& Allessie, M. A. Atrial fibrillation begets atrial fibrillation. a study in awake chronically instrumented goats. Circulation 92, 1954-1968 (1995).

468. Ausma, J. et al. Structural changes of atrial myocardium due to sustained atrial fibrillation in the goat. Circulation 96, 3157-3163 (1997).

469. Liu, L. et al. Exosomal miR-320d derived from adipose tissue-derived MSCs inhibits apoptosis in cardiomyocytes with atrial fibrillation (AF). Artif. Cells Nanomed. Biotechnol. 47, 3976-3984 (2019).

470. Wang, S. et al. Effects of atrial fibrillation-derived exosome delivery of miR-107 to human umbilical vein endothelial cells. DNA Cell Biol. 40, 568-579 (2021).

471. Li, S. et al. Myofibroblast-derived exosomes contribute to development of a susceptible substrate for atrial fibrillation. Cardiology 145, 324-332 (2020).

472. Cogswell, J. P. et al. Identification of miRNA changes in Alzheimer's disease brain and CSF yields putative biomarkers and insights into disease pathways. J. Alzheimers Dis. 14, 27-41 (2008).

473. Faghihi, M. A. et al. Expression of a noncoding RNA is elevated in Alzheimer's disease and drives rapid feed-forward regulation of beta-secretase. Nat. Med 14 723-730 (2008).

474. Hébert, S. S. et al. Loss of microRNA cluster miR-29a/b-1 in sporadic Alzheimer's disease correlates with increased BACE1/beta-secretase expression. Proc. Natl Acad. Sci. USA 105, 6415-6420 (2008).

475. Packer, A. N. et al. The bifunctional microRNA miR-9/miR-9* regulates REST and CoREST and is downregulated in Huntington's disease. J. Neurosci. 28, 14341-14346 (2008). 
476. Saba, R. et al. A miRNA signature of prion induced neurodegeneration. PLOS ONE 3, e3652 (2008).

477. Sonntag, K.-C. MicroRNAs and deregulated gene expression networks in neurodegeneration. Brain Res. 1338, 48-57 (2010).

478. Wang, W.-X. et al. The expression of microRNA miR-107 decreases early in Alzheimer's disease and may accelerate disease progression through regulation of $\beta$-site amyloid precursor protein-cleaving enzyme 1. J. Neurosci. 28, 1213-1223 (2008).

479. Sun, B.-L. et al. Clinical research on Alzheimer's disease: progress and perspectives. Neurosci. Bull. 34, 1111-1118 (2018).

480. Chen, J.-J. et al. Exosome-encapsulated microRNAs as promising biomarkers for Alzheimer's disease. Rev. Neurosci. 31, 77-87 (2019).

481. Leidinger, P. et al. A blood based 12-miRNA signature of Alzheimer disease patients. Genome Biol. 14, R78 (2013).

482. Chen, J.-J., Zhao, B., Zhao, J. \& Li, S. Potential roles of exosomal microRNAs as diagnostic biomarkers and therapeutic application in Alzheimer's disease. Neural Plast. 2017, 7027380 (2017).

483. Miya Shaik, M. et al. The Role of microRNAs in Alzheimer's disease and their therapeutic potentials. Genes (Basel). 9, 174 (2018).

484. Zhu, H.-C. et al. MicroRNA-195 downregulates Alzheimer's disease amyloid- $\beta$ production by targeting BACE1. Brain Res Bull. 88, 596-601 (2012).

485. Absalon, S., Kochanek, D. M., Raghavan, V. \& Krichevsky, A. M. MiR-26b, upregulated in Alzheimer's disease, activates cell cycle entry, tau-phosphorylation, and apoptosis in postmitotic neurons. J. Neurosci. 33, 14645-14659 (2013).

486. Jian, C. et al. miR-34a knockout attenuates cognitive deficits in APP/PS1 mice through inhibition of the amyloidogenic processing of APP. Life Sci. 182, 104-111 (2017).

487. $\mathrm{Xu}$, Y. et al. miR-34a deficiency in APP/PS1 mice promotes cognitive function by increasing synaptic plasticity via AMPA and NMDA receptors. Neurosci. Lett. 670, 94-104 (2018).

488. Sarkar, S. et al. Expression of microRNA-34a in Alzheimer's disease brain targets genes linked to synaptic plasticity, energy metabolism, and resting state network activity. Brain Res 1646, 139-151 (2016).

489. Liu, C.-G., Song, J., Zhang, Y.-Q. \& Wang, P.-C. MicroRNA-193b is a regulator of amyloid precursor protein in the blood and cerebrospinal fluid derived exosomal microRNA-193b is a biomarker of Alzheimer's disease. Mol. Med Rep. 10, 2395-2400 (2014).

490. Santos, J. R., Gois, A. M., Mendonça, D. M. F. \& Freire, M. A. M. Nutritional status, oxidative stress and dementia: The role of selenium in Alzheimer's disease. Front Aging Neurosci. 6, 206 (2014)

491. Kanamaru, T. et al. Oxidative stress accelerates amyloid deposition and memory impairment in a double-transgenic mouse model of Alzheimer's disease. Neurosci. Lett. 587, 126-131 (2015).

492. Kamat, P. K. et al. Mechanism of oxidative stress and synapse dysfunction in the pathogenesis of Alzheimer's disease: Understanding the therapeutics strategie. Mol. Neurobiol. 53, 648-661 (2016).

493. Cai, Z.-Y., Xiao, M., Quazi, S. H. \& Ke, Z.-Y. Exosomes: a novel therapeutic target for Alzheimer's disease. Neural Regen. Res 13, 930-935 (2018).

494. Lugli, G. et al. Plasma exosomal miRNAs in persons with and without Alzheimer disease: altered expression and prospects for biomarkers. PLOS ONE 10, e0139233 (2015)

495. Gayen, M., Bhomia, M., Balakathiresan, N. \& Knollmann-Ritschel, B. Exosomal microRNAs released by activated astrocytes as potential neuroinflammatory biomarkers. Int J. Mol. Sci. 21, 2312 (2020)

496. Ji, J. et al. Mitochondria-related miR-141-3p contributes to mitochondrial dysfunction in HFD-induced obesity by inhibiting PTEN. Sci. Rep. 5, 16262 (2015).

497. McKeever, P. M. et al. MicroRNA expression levels are altered in the cerebrospinal fluid of patients with young-onset Alzheimer's disease. Mol. Neurobiol. 55, 8826-8841 (2018).

498. Banzhaf-Strathmann, J. et al. MicroRNA-125b induces tau hyperphosphorylation and cognitive deficits in Alzheimer's disease. EMBO J. 33, 1667-1680 (2014).

499. Shen, Y. et al. MiR-125b-5p is involved in oxygen and glucose deprivation injury in PC-12 cells via CBS/H 2 S pathway. Nitric Oxide 78, 11-21 (2018)

500. Fotuhi, S. N., Khalaj-Kondori, M., Hoseinpour Feizi, M. A. \& Talebi, M. Long noncoding RNA BACE1-AS may serve as an Alzheimer's disease blood-based biomarker. J. Mol. Neurosci. 69, 351-359 (2019).

501. Kalia, L. V. \& Lang, A. E. Parkinson's disease. Lancet 386, 896-912 (2015).

502. Pagonabarraga, J., Kulisevsky, J., Strafella, A. P. \& Krack, P. Apathy in Parkinson's disease: clinical features, neural substrates, diagnosis, and treatment. Lancet Neurol. 14, 518-531 (2015)

503. Mirzaei, H. et al. Diagnostic and therapeutic potential of exosomes in cancer: the beginning of a new tale? J. Cell Physiol. 232, 3251-3260 (2017).

504. Li, D. et al. Effect of regulatory network of exosomes and microRNAs on neurodegenerative diseases. Chin. Med J. (Engl.) 131, 2216-2225 (2018).
505. Saeedi Borujeni, M. J. et al. Molecular aspects of diabetes mellitus: resistin, microRNA, and exosome. J. Cell Biochem. 119, 1257-1272 (2018).

506. Pourhanifeh, M. H. et al. MicroRNAs and exosomes: small molecules with big actions in multiple myeloma pathogenesis. IUBMB Life 72, 314-333 (2020).

507. Harischandra, D. S. et al. Environmental neurotoxicant manganese regulates exosome-mediated extracellular miRNAs in cell culture model of Parkinson's disease: relevance to a-synuclein misfolding in metal neurotoxicity. Neurotoxicology 64, 267-277 (2018).

508. Gui, Y. et al. Altered microRNA profiles in cerebrospinal fluid exosome in Parkinson disease and Alzheimer disease. Oncotarget 6, 37043-37053 (2015).

509. Asikainen, S. et al. Global microRNA expression profiling of Caenorhabditis elegans Parkinson's disease models. J. Mol. Neurosci. 41, 210-218 (2010).

510. Leggio, L. et al. microRNAs in Parkinson's disease: from pathogenesis to novel diagnostic and therapeutic approaches. Int J. Mol. Sci. 18, 2698 (2017).

511. Winkler, C. W., Taylor, K. G. \& Peterson, K. E. Location is everything: let-7b microRNA and TLR7 signaling results in a painful TRP. Sci. Signal. 7, pe14 (2014).

512. Shamsuzzama, Kumar, L. \& Nazir, A. Modulation of alpha-synuclein expression and associated effects by MicroRNA Let-7 in transgenic C. elegans. Front Mol. Neurosci. 10, 328 (2017).

513. Cao, X.-Y. et al. MicroRNA biomarkers of Parkinson's disease in serum exosomelike microvesicles. Neurosci. Lett. 644, 94-99 (2017).

514. Yao, Y. F. et al. Circulating exosomal miRNAs as diagnostic biomarkers in Parkinson's disease. Eur. Rev. Med Pharm. Sci. 22, 5278-5283 (2018).

515. Quinlan, S. et al. MicroRNAs in neurodegenerative diseases. Int Rev. Cell Mol. Biol. 334, 309-343 (2017).

516. Li, N. et al. Plasma levels of miR-137 and miR-124 are associated with Parkinson's disease but not with Parkinson's disease with depression. Neurol. Sci. 38, 761-767 (2017).

517. Jiang, Y. et al. Serum secreted miR-137-containing exosomes affects oxidative stress of neurons by regulating OXR1 in Parkinson's disease. Brain Res. 1722, 146331 (2019).

518. Wang, Q. et al. Integrated analysis of exosomal IncRNA and mRNA expression profiles reveals the involvement of Inc-MKRN2-42:1 in the pathogenesis of Parkinson's disease. CNS Neurosci. Ther. 26, 527-537 (2020).

519. Zou, J. et al. Long noncoding RNA POU3F3 and a-synuclein in plasma L1CAM exosomes combined with $\beta$-glucocerebrosidase activity: potential predictors of Parkinson's disease. Neurotherapeutics 17, 1104-1119 (2020).

520. Elkouris, M. et al. Long non-coding RNAs associated with neurodegenerationlinked genes are reduced in Parkinson's Disease patients. Front Cell Neurosci. 13, 58 (2019).

521. Scott, D. L., Wolfe, F. \& Huizinga, T. W. J. Rheumatoid arthritis. Lancet 376, 1094-1108 (2010).

522. Karsdal, M. A. et al. Cartilage degradation is fully reversible in the presence of aggrecanase but not matrix metalloproteinase activity. Arthritis Res Ther. 10, R63 (2008).

523. Wang, C.-h et al. CD147 induces angiogenesis through a vascular endothelial growth factor and hypoxia-inducible transcription factor 1a-mediated pathway in rheumatoid arthritis. Arthritis Rheum. 64, 1818-1827 (2012).

524. Sounni, N. E. et al. Stromal regulation of vessel stability by MMP14 and TGFbeta. Dis. Model Mech. 3, 317-332 (2010).

525. Ghisi, M. et al. Modulation of microRNA expression in human T-cell development: targeting of NOTCH3 by miR-150. Blood 117, 7053-7062 (2011).

526. Li, J. et al. Microvesicle-mediated transfer of microRNA-150 from monocytes to endothelial cells promotes angiogenesis. J. Biol. Chem. 288, 23586-23596 (2013).

527. Chen, Z. et al. Therapeutic potential of mesenchymal cell-derived miRNA-150$5 p$-expressing exosomes in rheumatoid arthritis mediated by the modulation of MMP14 and VEGF. J. Immunol. 201, 2472-2482 (2018).

528. Wang, Y. et al. MiR-548a-3p regulates inflammatory response via TLR4/NF-KB signaling pathway in rheumatoid arthritis. J Cell Biochem 120, 1113-1140 (2018).

529. Song, J. et al. PBMC and exosome-derived Hotair is a critical regulator and potent marker for rheumatoid arthritis. Clin. Exp. Med 15, 121-126 (2015).

530. Long, $\mathrm{H}$. et al. Dysregulation of microRNAs in autoimmune diseases: pathogenesis, biomarkers and potential therapeutic targets. Cancer Lett. 428, 90-103 (2018).

531. Le, X., Yu, X. \& Shen, N. Novel insights of microRNAs in the development of systemic lupus erythematosus. Curr. Opin. Rheumatol. 29, 450-457 (2017).

532. Salvi, V. et al. Exosome-delivered microRNAs promote IFN-a secretion by human plasmacytoid DCs via TLR7. JCI Insight 3, e98204 (2018).

533. Wang, P. et al. Inducible microRNA-155 feedback promotes type I IFN signaling in antiviral innate immunity by targeting suppressor of cytokine signaling 1. J. Immunol. 185, 6226-6233 (2010).

534. Aboelenein, H. R. et al. Reduction of CD19 autoimmunity marker on B cells of paediatric SLE patients through repressing PU.1/TNF-a/BAFF axis pathway by miR-155. Growth Factors 35, 49-60 (2017). 
535. Tyrrell, D. J. et al. Age-associated mitochondrial dysfunction accelerates atherogenesis. Circ. Res. 126, 298-314 (2020).

536. Xin, Q. et al. miR-155 deficiency ameliorates autoimmune inflammation of systemic lupus erythematosus by targeting S1pr1 in Faslpr/Ipr mice. J. Immunol. 194, 5437-5445 (2015).

537. Tang, Z. M. et al. Clinical relevance of plasma miR-21 in new-onset systemic lupus erythematosus patients. J. Clin. Lab Anal. 28, 446-451 (2014).

538. Stagakis, E. et al. Identification of novel microRNA signatures linked to human lupus disease activity and pathogenesis: miR-21 regulates aberrant $\mathrm{T}$ cell responses through regulation of PDCD4 expression. Ann. Rheum. Dis. 70, 1496-1506 (2011).

539. Tan, L. et al. Downregulated serum exosomal miR-451a expression correlates with renal damage and its intercellular communication role in systemic lupus erythematosus. Front Immunol. 12, 630112 (2021).

540. Dong, C. et al. Circulating exosomes derived-miR-146a from systemic lupus erythematosus patients regulates senescence of mesenchymal stem cells. Biomed. Res. Int. 2019, 6071308 (2019).

541. Perez-Hernandez, J. et al. Urinary exosomal miR-146a as a marker of albuminuria, activity changes and disease flares in lupus nephritis. J Nephrol. -, Aug 16. Online ahead of print, (2020).

542. Tsokos, G. C., Lo, M. S., Costa Reis, P. \& Sullivan, K. E. New insights into the immunopathogenesis of systemic lupus erythematosus. Nat. Rev. Rheumatol. 12, 716-730 (2016).

543. Santiago-Dieppa, D. R. et al. Extracellular vesicles as a platform for 'liquid biopsy' in glioblastoma patients. Expert Rev. Mol. Diagn. 14, 819-825 (2014).

544. Chun-Yan, L. et al. Liquid biopsy biomarkers of renal interstitial fibrosis based on urinary exosome. Exp. Mol. Pathol. 105, 223-228 (2018).

545. Gholaminejad, A., Abdul Tehrani, H. \& Gholami Fesharaki, M. Identification of candidate microRNA biomarkers in renal fibrosis: a meta-analysis of profiling studies. Biomarkers 23, 713-724 (2018).

546. Perez-Hernandez, J. et al. Increased urinary exosomal micrornas in patients with systemic lupus erythematosus. PLOS ONE 10, e0138618 (2015).

547. Zhou, $H$. et al. miR-150 promotes renal fibrosis in lupus nephritis by downregulating SOCS1. J. Am. Soc. Nephrol. 24, 1073-1087 (2013).

548. Liu, D. et al. miR-410 suppresses the expression of interleukin-6 as well as renal fibrosis in the pathogenesis of lupus nephritis. Clin. Exp. Pharm. Physiol. 43, 616-625 (2016).

549. Solé, C. et al. miR-29c in urinary exosomes as predictor of early renal fibrosis in lupus nephritis. Nephrol. Dial. Transpl. 30, 1488-1496 (2015).

550. Tangtanatakul, P. et al. Down-regulation of let-7a and miR-21 in urine exosomes from lupus nephritis patients during disease flare. Asian Pac. J. Allergy Immunol. 37, 189-197 (2019).

551. Hashemian, S. M. et al. Non-coding RNAs and exosomes: their role in the pathogenesis of sepsis. Mol. Ther. Nucleic Acids 21, 51-74 (2020).

552. $\mathrm{Wu}, \mathrm{X}$. et al. Serum exosomal MicroRNAs predict acute respiratory distress syndrome events in patients with severe community-acquired pneumonia. Biomed. Res. Int. 2019, 3612020 (2019).

553. Huang, F. et al. Identification of potential diagnostic biomarkers for pneumonia caused by adenovirus infection in children by screening serum exosomal microRNAs. Mol. Med Rep. 19, 4306-4314 (2019).

554. Torres, A. et al. Challenges in severe community-acquired pneumonia: a pointof-view review. Intensive Care Med. 45, 159-171 (2019).

555. Jiang, K. et al. Peripheral circulating exosome-mediated delivery of miR-155 as a novel mechanism for acute lung inflammation. Mol. Ther. 27, 1758-1771 (2019).

556. Quan, Y. et al. Exosome miR-371b-5p promotes proliferation of lung alveola progenitor type II cells by using PTEN to orchestrate the PI3K/Akt signaling. Stem Cell Res Ther. 8, 138 (2017).

557. Zhu, Z. et al. Macrophage-derived apoptotic bodies promote the proliferation of the recipient cells via shuttling microRNA-221/222. J. Leukoc. Biol. 101, 1349-1359 (2017).

558. Zhang, D. et al. A potential role of microvesicle-containing miR-223/142 in lung inflammation. Thorax 74, 865-874 (2019).

559. Li, R., Liang, P., Yuan, J. \& He, F. Exosomal miR-103a-3p ameliorates lipopolysaccharide-induced immune response in BEAS-2B cells via NF-kappaB pathway by targeting transducin beta-like $1 \mathrm{X}$ related protein 1. Clin. Exp. Pharm. Physiol. 47, 620-627 (2020).

560. Jiao, Y. et al. Advances on liver cell-derived exosomes in liver diseases. J. Cell Mol. Med. 25, 15-26 (2021).

561. Khatun, M. \& Ray, R. B. Mechanisms underlying hepatitis $C$ virus-associated hepatic fibrosis. Cells 8, 1249 (2019).

562. Bandopadhyay, M. \& Bharadwaj, M. Exosomal miRNAs in hepatitis B virus related liver disease: a new hope for biomarker. Gut Pathog. 12, 23 (2020).

563. Li, R. et al. Expression profiles of the exosomal miRNAs in the chronic hepatitis $B$ patients with persistently normal ALT. Zhong Nan Da Xue Xue Bao Yi Xue Ban. 43 , 475-480 (2018).

564. Devhare, P. B. et al. xosome-mediated intercellular communication between hepatitis C virus-infected hepatocytes and hepatic stellate Cells.J Virol. 91, e02225-16 (2017).

565. Thakuri, B. K. C. et al. HCV-Associated exosomes upregulate RUNXOR and RUNX1 expressions to promote MDSC expansion and suppressive functions through STAT3-miR124 Axis. Cells 9, 2715 (2020).

566. Liu, C., Gao, Y., Wu, J. \& Zou, J. Exosomal miR-23a and miR-192, potential diagnostic biomarkers for Type 2 diabetes. Clin. Laboratory. 67, https://doi.org/ 10.7754/clin.lab.2020.200612 (2021).

567. Fu, Q. et al. Injury factors alter miRNAs profiles of exosomes derived from islets and circulation. Aging 10, 3986-3999 (2018).

568. Shao, J.-L. et al. Identification of serum exosomal MicroRNA expression profiling in menopausal females with osteoporosis by high-throughput sequencing. Curr. Med. Sci. 40, 1161-1169 (2020).

569. Karolina, D. S. et al. Circulating miRNA profiles in patients with metabolic syndrome. J. Clin. Endocrinol. Metab. 97, E2271-E2276 (2012).

570. Mendt, M. et al. Generation and testing of clinical-grade exosomes for pancreatic cancer. JCl Insight. 3, e99263 (2018).

571. Alvarez-Erviti, L. et al. Delivery of siRNA to the mouse brain by systemic injection of targeted exosomes. Nat. Biotechnol. 29, 341-345 (2011).

572. Tang, T. T., Wang, B., Lv, L. L. \& Liu, B. C. Extracellular vesicle-based nanotherapeutics: emerging frontiers in anti-inflammatory therapy. Theranostics 10 8111-8129 (2020)

573. Lv, Q. et al. Engineered human adipose stem-cell-derived exosomes loaded with miR-21-5p to promote diabetic cutaneous wound healing. Mol. pharmaceutics 17, 1723-1733 (2020)

574. Kim, G. et al. Systemic delivery of microRNA-21 antisense oligonucleotides to the brain using T7-peptide decorated exosomes. J. Control Release 317, 273-281 (2020).

575. Cheng, L. et al. Characterization and deep sequencing analysis of exosomal and non-exosomal miRNA in human urine. Kidney Int. 86, 433-444 (2014).

576. Nik Mohamed Kamal, N. \& Shahidan, W. N. S. Non-exosomal and exosomal circulatory MicroRNAs: which are more valid as biomarkers? Front Pharm. 10, 1500 (2019).

577. Zhao, C., Busch, D. J., Vershel, C. P. \& Stachowiak, J. C. Multifunctional transmembrane protein ligands for cell-specific targeting of plasma membranederived vesicles. Small 12, 3837-3848 (2016).

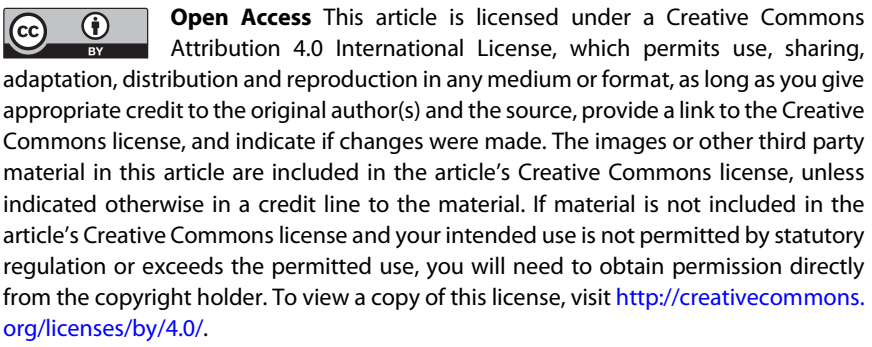

(c) The Author(s) 2021 